\title{
Solving Embedding Problems With BOUNDED RAMIFICATION
}

\author{
Nantsoina Cynthia Ramiharimanana
}

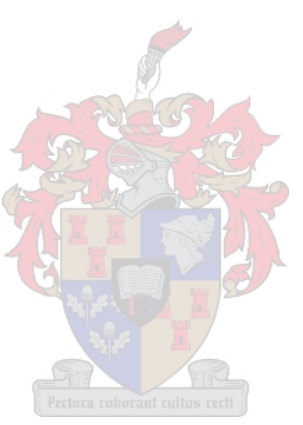

Dissertation presented for the degree of Doctor of Philosophy in Mathematics at Stellenbosch University

Promoters: Prof. Moshe Jarden Prof. Barry Green

(Tel Aviv University) (Stellenbosch University)

December 2016 


\section{Stellenbosch University https://scholar.sun.ac.za}




\section{Declaration}

By submitting this thesis electronically, I declare that the entirety of the work contained therein is my own, original work, that I am the owner of the copyright thereof (unless to the extent explicitly otherwise stated) and that I have not previously in its entirety or in part submitted it for obtaining any qualification.

Date: ........... December 2016

Signature: .......................................

Copyright (C)2016 Stellenbosch University

All rights reserved 


\section{Abstract}

Given a Galois extension $K / K_{0}$ of number fields, a finite group $G$, and an epimorphism $\alpha: G \longrightarrow \operatorname{Gal}\left(K / K_{0}\right)$ with solvable kernel, our goal is to embed $K$ into a Galois extension $N$ of $K_{0}$ with Galois group $\operatorname{Gal}\left(N / K_{0}\right) \cong G$ such that the restriction map $\operatorname{res}_{N / K}: \operatorname{Gal}\left(N / K_{0}\right) \longrightarrow \operatorname{Gal}\left(K / K_{0}\right)$ coincides with $\alpha$ and $\left|\operatorname{Ram}\left(N / K_{0}\right)\right| \leq\left|\operatorname{Ram}\left(K / K_{0}\right)\right|+\Omega(|\operatorname{Ker}(\alpha)|)$. Here $\operatorname{Ram}\left(N / K_{0}\right)$ is the finite set of primes of $K_{0}$ that ramify in $N$ and $\Omega(|\operatorname{Ker}(\alpha)|)$ is the number of the prime divisors, counted with multiplicity, of $|\operatorname{Ker}(\alpha)|$.

We achieve our goal under two conditions: first, the number of roots of unity in $K$ should be relatively prime to the order of $\operatorname{Ker}(\alpha)$. The second one demands that each local embedding problem resulted from the original one should be "weakly solvable". In fact, our solution locally coincides with finitely many "local weak solutions" given in advance.

Our result strengthens a former result of Neukirch in [Neu79, where the same embedding problem satisfying the same conditions is solved without giving a bound on the ramification.

In particular, the above mentioned local conditions are satisfied if the epimorphism $\alpha$ has a section. This leads to a well known result of Shafarevich that does not assume the condition on the roots of unity but pays with a huge number of ramified primes (that appears when one analyses Shafarevich's proof).

Like in [Neu79], our proof uses class field theory in its cohomological approach. The bounding of the ramification is based, in addition to the above mentioned tools, on a strengthening of a lemma of GeJ98. 


\section{Opsomming}

Laat $K / K_{0}$ 'n Galois uitbreiding van getalleliggame wees, $G$ 'n eindige groep, en $\alpha: G \longrightarrow \operatorname{Gal}\left(K / K_{0}\right)$ 'n epimorfisme met oplosbare kern. Ons doel is om $K$ in 'n Galois uitbreiding $N$ van $K_{0}$ in-te-bed sodat die Galois groep $\operatorname{Gal}\left(N / K_{0}\right) \cong G$, en sodat die beperkingsafbeelding $\operatorname{res}_{N / K}: \operatorname{Gal}\left(N / K_{0}\right) \longrightarrow$ $\operatorname{Gal}\left(K / K_{0}\right)$ ooreenstem met $\alpha$ en $\left|\operatorname{Ram}\left(N / K_{0}\right)\right| \leq\left|\operatorname{Ram}\left(K / K_{0}\right)\right|+\Omega(|\operatorname{Ker}(\alpha)|)$. Hier is $\operatorname{Ram}\left(N / K_{0}\right)$ die eindige versameling van priemdelers van $K_{0}$ wat in $N$ vertak, en $\Omega(|\operatorname{Ker}(\alpha)|)$ is die aantal priemdelers van $|\operatorname{Ker}(\alpha)|$, getel met multiplisiteit.

Ons bereik hierdie doelstelling onderhewig aan twee voorwaardes: Eerstens moet die aantal wortels van eenheid in $K$ relatief priem wees aan die orde van $\operatorname{Ker}(\alpha)$. Tweedens eis ons dat elke lokale inbeddingsprobleem, wat volg uit die oorspronklike een, "swak oplosbaar" moet wees. Meer presies gestel, sal ons oplossing lokaal ooreenstem met 'n eindige aantal "lokaal swak oplossings" wat vooraf gegee word.

Ons resultaat versterk 'n vroeër resultaat van Neukirch in [Neu79], waar 'n inbeddingsprobleem wat dieselfde voorwaardes bevredig opgelos word, maar sonder die grens op die aantal vertakkings.

In die besonder word die lokale voorwaardes bevredig mits die epimorfisme $\alpha$ 'n snitafbeelding besit. Hieruit volg dan ook die bekende resultaat van Shafarevich, wat nie die voorwaarde oor die wortels van eenheid benodig nie, maar gevolglik 'n baie groot aantal priemdelers wat vertak veroorsaak (hierdie opmerking word gesien wanneer sy bewys in detail bestudeer word).

Soos in [Neu79], maak ons gebruik van klasliggaamteorie met 'n kohomologiese benadering. Die begrensdheid van die aantal priemdelers wat vertak maak ook gebruik van 'n versterking van 'n hulpstelling uit GeJ98. 


\section{Acknowledgements}

I would like to express my sincere gratitude to my advisors Prof. Moshe Jarden and Prof. Barry Green for giving me the opportunity to continue my studies and to work on this project. They allowed me to grow as a researcher scientist. Their incredible assistance, support and guidance made this thesis possible. Special thanks go to Prof. Moshe Jarden for his patience with my questions, for our valuable discussions, and for his constructive comments that enormously strengthened and improved this work.

I am thankful to Prof. Wulf-Dieter Geyer, Prof. Kay Wingberg, and Dr. Sebastian Petersen for their useful comments on some materials that I used in this work (private communication). I am also grateful to Prof. LiorBarry Soroker, Mrs Meira Hilel, and Mrs Rina Jarden for their valuable assistance during my visit at the School of Mathematics at Tel Aviv University. Thank you to DAAD (Deutscher Akademischer Austausch Dienst), to AIMS-SA (African Institute for Mathematical Sciences - South Africa), to the Mathematical Department at Stellenbosch University, and to the School of Mathematics at Tel Aviv University for their financial and material support throughout this work.

I thank and praise the almighty God for proving me strength to proceed successfully. I cordially thank my family (Dad, Mom, sisters and brother) for their deepest love, support and patience during my studies. I always felt their presence even if I was far away from home. "Thank you" to my LePisou for sharing with me courage, love and wonderful way of living life. 
This thesis is dedicated to my parents. 


\section{Contents}

Declaration i

Abstract ii

Opsomming iii

Acknowledgements iv

Notation viii

\begin{tabular}{ll}
\hline Introduction & 1
\end{tabular}

$\begin{array}{lll}1 & \text { Preliminaries } & 9\end{array}$

1.1 Topological group homomorphisms $\ldots \ldots \ldots$. . . . . . . 9

1.2 Embedding problems and ramification $\ldots \ldots \ldots \ldots$

1.3 A basic set of primes and the reciprocity law . . . . . . . 17

1.4 Cohomology groups and special mappings . . . . . . . . 22

$\begin{array}{|ll|}2 \text { Bound on the ramification of homomorphisms } & 28\end{array}$

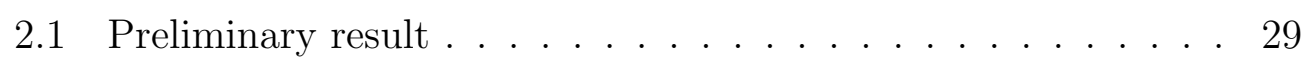

2.2 Isomorphism of $\operatorname{Hom}(\operatorname{Gal}(K), A)$ and $\operatorname{Hom}\left(C_{K}, A\right) \ldots \ldots$

$2.3 \quad$ Construction of $h \in \operatorname{Hom}\left(\operatorname{Gal}(K), C_{l}\right) \ldots \ldots \ldots$

2.4 Bound on the ramification of $h \in \operatorname{Hom}(\operatorname{Gal}(K), A) \ldots \ldots$

3 Bound on the ramification of cohomology classes 45

3.1 Definitions and a preliminary result . . . . . . . . . . 46 
3.2 Corestriction map . . . . . . . . . . . . . . . . . . . . . . . . . 47

3.3 Bound on the ramification of 1-cocycles . . . . . . . . . . . 53

4 Solving embedding problems with bounded ramification 61

4.1 The principal homogeneous space over $H^{1}\left(\operatorname{Gal}\left(K_{0}\right), A\right)$. . . . 62

4.2 Embedding problem whose kernel is a simple $\mathrm{Gal}\left(K_{0}\right)$-module 66

$4.3 \quad$ Embedding problem with solvable kernel . . . . . . . . . . . . 73

4.4 Solving embedding problems with solvable kernels in $K_{0, \text { tot }, S}$. 78

\begin{tabular}{lr}
\hline Bibliography & 83
\end{tabular} 


\section{Notation}

$K=$ a number field.

$\tilde{K}=$ a fixed algebraic closure of $K$.

$\operatorname{irr}(x, K)=$ the monic irreducible polynomial over $K$ of an element $x \in \tilde{K}$

$\operatorname{Gal}(L / K)=$ the Galois group of a Galois extension $L / K$.

$\operatorname{Gal}(K)=\operatorname{Gal}(\tilde{K} / K)=$ the absolute Galois group of $K$.

$\operatorname{res}_{L / K}=$ the restriction map $\operatorname{Gal}\left(L / K_{0}\right) \rightarrow \operatorname{Gal}\left(K / K_{0}\right)$ for a tower of Galois extensions $K_{0} \subseteq K \subseteq L$.

$\mathbb{P}(K)=$ the set of all primes of $K$.

$\mathbb{P}_{\text {fin }}(K)=$ the set of all finite primes of $K$.

$\mathbb{P}_{\infty}(K)=$ the set of infinite primes of $K$.

$\hat{K}_{\mathfrak{p}}=$ a fixed completion of $K$ at $\mathfrak{p}$.

$K_{\mathfrak{p}}=$ a fixed henselization of $K$ at $\mathfrak{p}$.

$\operatorname{Ram}(L / K)=$ the finite set of primes of $K$ which ramify in $L$.

$I(L / K)=$ the inertia group of a Galois extension of local fields $L / K$ of characteristic zero.

$\zeta_{n}=$ a fixed $n$-th primitive root of unity in $\tilde{K}$ for a positive integer $n$.

$\mu(K)=$ the set of roots of unity in the field $K$.

$l=$ a variable for prime numbers.

$C_{l}=$ the multiplicative cyclic group of order $l$.

$\hat{\mathbb{Z}}=\lim \mathbb{Z} / n \mathbb{Z}=$ the Prüfer group.

$\bigcup_{i=1}^{n} A_{i}=$ the disjoint union of sets $A_{1}, \ldots, A_{n}$. 


\section{Introduction}

A sharpened version of the inverse Galois problem is the so called embedding problem. Given a Galois extension $K / K_{0}$ of number fields, a finite group $G$, and an epimorphism $\alpha: G \rightarrow \operatorname{Gal}\left(K / K_{0}\right)$, one looks for a Galois extension $N$ of $K_{0}$ that contains $K$ such that $\operatorname{Gal}\left(N / K_{0}\right) \cong G$ and the restriction map $\operatorname{res}_{N / K}: \operatorname{Gal}\left(N / K_{0}\right) \rightarrow \operatorname{Gal}\left(K / K_{0}\right)$ coincides with $\alpha$. Equivalently, one looks for a continuous epimorphism $\psi: \operatorname{Gal}\left(K_{0}\right) \rightarrow G$ such that $\alpha \circ \psi=\operatorname{res}_{\tilde{K}_{0} / K}$. We refer to $\psi$ as a proper solution of the embedding problem (whereas, if $\psi$ is only a homomorphism as above, we say that $\psi$ is a solution of the embedding problem). The fixed field $N$ of $\operatorname{Ker}(\psi)$ in $\tilde{K}_{0}$ is the solution field of the embedding problem. The question about the proper solvability of finite embedding problems is of course far from being settled. But, in those cases where an embedding problem as above is solvable, we ask whether a solution field $N$ can be found with a bound on the ramification, i.e. bound on the number of the primes of $K_{0}$ that are ramified in $N$.

PREVIOUS WORKS. The combinatorial arguments of Shafarevich in Sha54 (which was corrected in Sha89] for $l=2$ ) lead for each finite $l$-group $G$ to a Galois extension $N$ of $K$ with Galois group $G$ such that $|\operatorname{Ram}(N / K)|$ has an exponential growth in $|G|$.

In [GeJ98, Geyer and Jarden use the method of Scholz [Sch37] and Reichardt [Rei37] in order to realize each l-group $G$ over a global field $K$ under the condition $\zeta_{l} \notin K$. If $|G|=l^{n}$, they construct a Galois extension $N$ of $K$ with $\operatorname{Gal}(N / K) \cong G$ and $|\operatorname{Ram}(N / K)| \leq n+r(K)$, where $r(K)$ depends only on arithmetical invariants of $K$. If $K=\mathbb{Q}$ or $K=\mathbb{F}_{q}(t)$, then $r(K)=0$, so the result of GeJ98 reproduces in this case the result of Serre in $[\mathrm{Se} 92$ that $|\operatorname{Ram}(N / K)| \leq n$.

The result of [GeJ98] is generalized by Markin and Ullom in [MaU11. The latter work constructs for each number field $K$ and every finite nilpotent group $G$ a Galois extension $N$ of $K$ with Galois group $G$. Moreover, if 
$\left(G_{i}\right)_{i}$ is a lower central series of $G$ and $d\left(G_{i} / G_{i+1}\right)$ is the minimal number of generators of $G_{i} / G_{i+1}$, then $|\operatorname{Ram}(N / K)| \leq \sum_{i} d\left(G_{i} / G_{i+1}\right)+r(K)$.

Going back to the case of a finite embedding problem $\alpha: G \rightarrow \operatorname{Gal}\left(K / K_{0}\right)$ for number fields, Neukirch observes in [Neu79] that for each prime divisor $\mathfrak{p}$ of $K_{0}$, the completion $\hat{K}_{\mathfrak{p}} / \hat{K}_{0, \mathfrak{p}}$ of $K / K_{0}$ at $\mathfrak{p}$ gives rise to a local embedding problem. In the spirit of Scholz-Reichardt, Neu79] proves that if $\operatorname{Ker}(\alpha)$ is solvable, $\operatorname{gcd}(|\operatorname{Ker}(\alpha)|,|\mu(K)|)=1$, and each of the local embedding problems is solvable, then the original global embedding problem is properly solvable. Moreover, one may find a proper solution that coincides on $\operatorname{Gal}\left(\hat{K}_{0, \mathfrak{p}}\right)$ with a given local solution $\varphi_{\mathfrak{p}}$ for finitely many $\mathfrak{p}$ 's. However, [Neu79] gives no bound on the ramification of the proper solution.

THE MAIN RESULT. It is exactly the latter gap that our work intends to fill out. To this end we recall that if $n=\prod_{i=1}^{m} l_{i}^{r_{i}}$ is the decomposition of a positive integer $n$ into a product of powers of distinct primes $l_{1}, \ldots, l_{m}$, then $\Omega(n)=\sum_{i=1}^{m} r_{i}$. For each $\mathfrak{p} \in \mathbb{P}\left(K_{0}\right)$, we identity $\operatorname{Gal}\left(\hat{K}_{0, \mathfrak{p}}\right)$ with $\operatorname{Gal}\left(K_{0, \mathfrak{p}}\right)$ as a subgroup of $\operatorname{Gal}\left(K_{0}\right)$. We prove the following result:

THEOREM A: Let $K / K_{0}$ be a finite Galois extension of number fields, set $\Gamma=\operatorname{Gal}\left(K / K_{0}\right)$ and consider a finite embedding problem

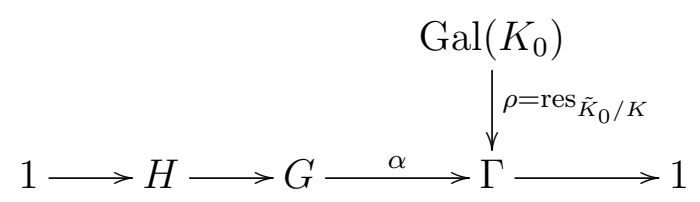

with a solvable kernel $H$. Suppose that

(a1) $\operatorname{gcd}(|H|,|\mu(K)|)=1$ and

(a2) for each $\mathfrak{p} \in \mathbb{P}\left(K_{0}\right)$ there exists a homomorphism $\psi_{\mathfrak{p}}$ : $\operatorname{Gal}\left(\hat{K}_{0, \mathfrak{p}}\right) \rightarrow G$ such that $\alpha \circ \psi_{\mathfrak{p}}=\left.\rho\right|_{\operatorname{Gal}\left(\hat{K}_{0, \mathfrak{p}}\right)}$ (we call $\psi_{\mathfrak{p}}$ a local solution).

Let $T$ be a finite set of primes of $K_{0}$ that contains $\operatorname{Ram}\left(K / K_{0}\right)$ and for each $\mathfrak{p} \in T$ let $\varphi_{\mathfrak{p}}$ be a local solution. Then, there exists an epimorphism 
$\psi: \operatorname{Gal}\left(K_{0}\right) \rightarrow G$ such that $\alpha \circ \psi=\rho$ and there exists a set $R \subset \mathbb{P}\left(K_{0}\right) \backslash T$ with $|R|=\Omega(|H|)$ that satisfy the following conditions

(b1) For each $\mathfrak{p} \in T$ there exists $a \in H$ such that $\psi(\sigma)=a^{-1} \varphi_{\mathfrak{p}}(\sigma) a$ for all $\sigma \in \operatorname{Gal}\left(\hat{K}_{0, \mathfrak{p}}\right)$.

(b2) The fixed field $N$ in $\tilde{K}_{0}$ of $\operatorname{Ker}(\psi)$ satisfies $\operatorname{Ram}\left(N / K_{0}\right) \subseteq T \cup R$, hence $\left|\operatorname{Ram}\left(N / K_{0}\right)\right| \leq|T|+\Omega(|H|)$.

SPECIAL CASES. Note that if the short exact sequence in 0.1 .1 splits, then the condition in Theorem A about the local solvability is automatically satisified. Thus in this case, Theorem A holds under the mere conditions that $H$ is solvable and $\operatorname{gcd}(|H|,|\mu(K)|)=1$.

Also, let $S$ be a finite subset of $\mathbb{P}\left(K_{0}\right)$ and denote the maximal Galois extension of $K_{0}$ in which each $\mathfrak{p} \in S$ totally splits by $K_{0, \text { tot }, S}$. Suppose that $K \subseteq K_{0, \text { tot, } S}$. Then, assume that $S \subseteq T$ and take $\varphi_{\mathfrak{p}}$ for each $\mathfrak{p} \in S$ as the trivial homomorphism. We find that the solution field $N$ of $(0.1 .1)$ is contained in $K_{0, \text { tot }, S}$.

Finally, we note that if $|G|=l^{n}$ is an $l$-group and if we take $K=K_{0}$, that is $\Gamma$ is trivial, Theorem $A$ gives a Galois extension $N$ of $K$ with Galois group $G$ such that $|\operatorname{Ram}(N / K)| \leq n$. This improves the estimate of the main result of GeJ98 mentioned above.

OUTLINE OF THE PROOF OF THEOREM A. We prove the theorem by induction on the order of $H$. We start with a reduction step.

Part 1: A reduction step. We choose a maximal proper subgroup $H_{1}$ of $H$ which is normal in $G$. This breaks up the embedding problem 0.1.1 into two embedding problems. This first one is

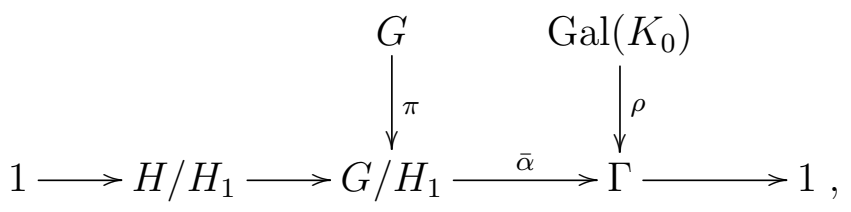


where $\bar{\alpha} \circ \pi=\alpha$ and $\pi: G \rightarrow G / H_{1}$ is the quotient map.

Part 2: Induction step. The second one appears as soon as we find a proper solution $\bar{\psi}: \operatorname{Gal}\left(K_{0}\right) \rightarrow G / H_{1}$ of embedding problem 0.1 .2 :

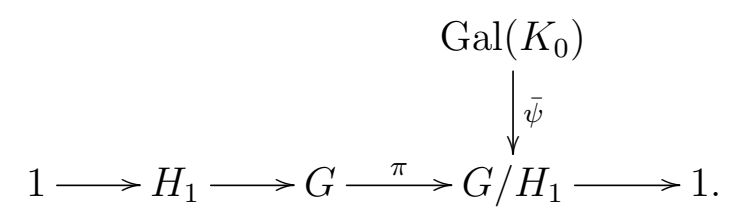

We have $\left|H_{1}\right|<|H|$. But in order to proceed to the induction, we assume that embedding problem 0.1 .3 satisfies in its objects conditions (a1) and (a2) of Theorem A. In particular

(c1) $\operatorname{gcd}\left(\left|H_{1}\right|,|\mu(\bar{K})|\right)=1$ where $\bar{K}$ is the fixed field of $\operatorname{Ker}(\bar{\psi})$ in $\tilde{K}_{0}$,

(c2) for each $\mathfrak{p} \in \mathbb{P}\left(K_{0}\right)$ there exists a homomorphism $\psi_{\mathfrak{p}}^{\prime}: \operatorname{Gal}\left(\hat{K}_{0, \mathfrak{p}}\right) \rightarrow G$ such that $\pi \circ \psi_{\mathfrak{p}^{\prime}}=\left.\bar{\psi}\right|_{\operatorname{Gal}\left(\hat{K}_{0, \mathfrak{p}}\right)}$, i.e. each local embedding problem is solvable.

The first step of the induction also supplies a finite subset $\bar{R}$ of $\mathbb{P}\left(K_{0}\right) \backslash T$ such that $\operatorname{Ram}\left(\bar{K} / K_{0}\right) \subseteq T \cup \bar{R}$ and $|\bar{R}|=\Omega\left(\left|H / H_{1}\right|\right)$.

Replacing $T$ in Theorem A by $T \cup \bar{R}$, an induction hypothesis on the order of the kernel gives a proper solution $\psi$ to embedding problem 0.1 .3 that satisfies conclusions (b1) and (b2) of Theorem A, again with respect to the objects associated with 0.1.3). In particular, if $N$ is the fixed field of $\operatorname{Ker}(\psi)$ in $\tilde{K}_{0}$, then there exists a subset $R_{1}$ of $\mathbb{P}\left(K_{0}\right) \backslash(T \cup \bar{R})$ such that $\left|R_{1}\right|=\Omega\left(\left|H_{1}\right|\right)$ and $\operatorname{Ram}\left(N / K_{0}\right) \subseteq T \cup \bar{R} \cup R_{1}$. Then, $\psi$ is a solution of 0.1 .1 that satisfies (b1) and (b2) for the original embedding problem 0.1.1). Setting $R=\bar{R} \cup R_{1}$, we have $\operatorname{Ram}\left(N / K_{0}\right) \subseteq T \cup R$ and $|R|=\Omega(|H|)$.

Part 3: The kernel is a simple module. The choice of $H_{1}$ in Part 1 implies that $H / H_{1}$ is a minimal normal subgroup of $G / H_{1}$. Since $H$ is solvable, there exist a prime number $l$ and a positive integer $r$ such that $A=H / H_{1} \cong C_{l}^{r}$. The action of $\bar{G}=G / H_{1}$ on $A$ by conjugation makes $A$ a simple multiplicative 
$\Gamma$-module (via lifting with $\alpha$ ), hence also a simple $\operatorname{Gal}\left(K_{0}\right)$-module (through $\rho)$. The next result strengthens the solvability with bounded ramification of Embedding problem 0.1 .2 such that Embedding problem 0.1 .3 satisfies the induction hypothesis.

PROPOSITION B: Let $K / K_{0}$ be a finite Galois extension of number fields, set $\Gamma=\operatorname{Gal}\left(K / K_{0}\right)$ and $\rho=\operatorname{res}_{\tilde{K}_{0} / K}$. Then consider the finite embedding problem:

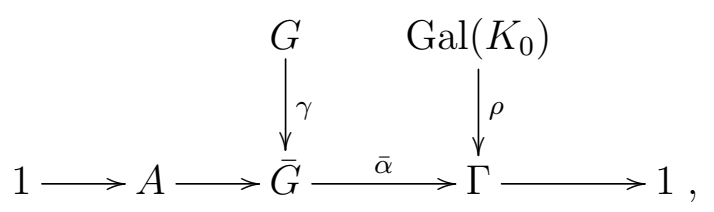

with $A \cong C_{l}^{r}$ a simple $\operatorname{Gal}\left(K_{0}\right)$-module and where $\gamma: G \rightarrow \bar{G}$ is an epimorphism of finite groups with a non-trivial solvable kernel. Let $n$ be a multiple of $l|\operatorname{Ker}(\gamma)|$. Suppose

$(\mathrm{d} 1) \operatorname{gcd}(n,|\mu(K)|)=1$,

(d2) for each $\mathfrak{p} \in \mathbb{P}\left(K_{0}\right)$ there exists a homomorphism $\psi_{\mathfrak{p}}: \operatorname{Gal}\left(\hat{K}_{0, \mathfrak{p}}\right) \rightarrow \bar{G}$ such that $\bar{\alpha} \circ \psi_{\mathfrak{p}}=\left.\rho\right|_{\operatorname{Gal}\left(\hat{K}_{0, \mathfrak{p}}\right)}$.

Let $T$ be a finite subset of $\mathbb{P}\left(K_{0}\right)$ that contains $\operatorname{Ram}\left(K / K_{0}\right)$ and for each $\mathfrak{p} \in$ $T$ let $\bar{\varphi}_{\mathfrak{p}}$ be a local solution. Then, there exists an epimorphism $\bar{\psi}: \operatorname{Gal}\left(K_{0}\right) \rightarrow$ $\bar{G}$ such that $\bar{\alpha} \circ \bar{\psi}=\rho$ and there exists a set $\bar{R} \subset \mathbb{P}\left(K_{0}\right) \backslash T$ with $|\bar{R}|=$ $\Omega(|A|)=r$ that satisfy the following conditions

(e1) For each $\mathfrak{p} \in T$ there exists $a \in A$ such that $\psi(\sigma)=a^{-1} \bar{\varphi}_{\mathfrak{p}}(\sigma) a$ for all $\sigma \in \operatorname{Gal}\left(\hat{K}_{0, \mathfrak{p}}\right)$.

(e2) The fixed field $\bar{K}$ in $\tilde{K}_{0}$ of $\operatorname{Ker}(\bar{\psi})$ satisfies $\operatorname{Ram}\left(\bar{K} / K_{0}\right) \subseteq T \cup \bar{R}$.

(e3) $\operatorname{gcd}(n,|\mu(\bar{K})|)=1$,

(e4) for each $\mathfrak{p} \in \mathbb{P}\left(K_{0}\right)$ there exists a homomorphism $\psi_{\mathfrak{p}}^{\prime}: \operatorname{Gal}\left(\hat{K}_{0, \mathfrak{p}}\right) \rightarrow G$ such that $\gamma \circ \psi_{\mathfrak{p}}^{\prime}=\left.\bar{\psi}\right|_{\operatorname{Gal}\left(\hat{K}_{0, \mathfrak{p}}\right)}$. 
Conditions (e3) and (e4) take care of the induction hypothesis (c1) and (c2) respectively.

Using Condition (d1) (which implies that $\zeta_{l} \notin K$ ), Condition (d2) and the assumption that $A$ is a simple $\operatorname{Gal}\left(K_{0}\right)$-module, we conclude from the local-global principle (Lemma 1.2.8) that there exists a homomorphism $\psi_{0}: \operatorname{Gal}\left(K_{0}\right) \rightarrow \bar{G}$ such that $\bar{\alpha} \circ \psi_{0}=\rho$. However, we still have to adjust $\psi_{0}$ such that it will be surjective, to find a subset $\bar{R}$ of $\mathbb{P}\left(K_{0}\right) \backslash T$ such that Condition (e2) holds, and to satisfy Condition (e4). In order to achieve those conditions, we follow [Neu79. We choose an appropriate crossed homomorphism $\chi: \operatorname{Gal}\left(K_{0}\right) \rightarrow A$, and consider the adjusted solution $\bar{\psi}=\psi_{0} \cdot \chi$ of 0.1 .4 .

In order to make $\bar{\psi}$ surjective, we use the Chebotarev density theorem in order to choose a prime $\mathfrak{q} \in \mathbb{P}\left(K_{0}\right) \backslash T$ that totally splits in $K\left(\zeta_{n}\right)$ and a cyclic unramified homomorphism $\bar{\varphi}_{\mathfrak{q}}: \operatorname{Gal}\left(\hat{K}_{0, \mathfrak{q}}\right) \rightarrow\langle a\rangle \leq A$ with $a \neq 1$, such that the solution $\bar{\psi}$ will satisfy $\left.\bar{\psi}\right|_{\operatorname{Gal}\left(\hat{K}_{0, \mathfrak{q}}\right)}=\bar{\varphi}_{\mathfrak{q}}$. This implies that $A \cap \operatorname{Im}(\bar{\psi}) \neq 1$. Since $A$ is a simple $\operatorname{Gal}\left(K_{0}\right)$-module, this together with the assumption that $\rho$ is surjective implies that $\operatorname{Im}(\bar{\psi})=\bar{G}$, that is $\bar{\psi}$ is a proper solution of 0.1 .3 (Lemma 4.2.1).

Moreover, the "ramification of $\chi$ " eliminates the "ramification of $\psi_{0}$ " up to a set $\bar{R}=\left\{\mathfrak{q}_{1}, \ldots, \mathfrak{q}_{r}\right\}$ that satisfies $\bar{R} \subset \mathbb{P}\left(K_{0}\right) \backslash T,|\bar{R}|=\Omega(|A|)$ and $\operatorname{Ram}\left(\bar{K} / K_{0}\right) \subseteq T \cup \bar{R}$, where $\bar{K}$ is the fixed field in $\tilde{K}_{0}$ of $\operatorname{Ker}(\bar{\psi})$. The primes $\mathfrak{q}_{i}$ are chosen by the Chebotarev density theorem. That theorem gives us freedom to choose the $\mathfrak{q}_{i}$ 's away from $T$ and away from the divisors of $l$. In addition, the $\mathfrak{q}_{i}$ 's are also chosen in such a way that $\left.\bar{\psi}\right|_{\operatorname{Gal}\left(\hat{K}_{0, q_{i}}\right)}$ is unramified or cyclically ramified.

Next we prove Condition (e4). The choice of the $\mathfrak{q}_{i}$ 's leaves us with three possibilities for each $\mathfrak{p} \in \mathbb{P}\left(K_{0}\right)$. The first possibility is that $\mathfrak{p} \in T$. In this case Condition (e1) and the properties of the homomorphism $\gamma$ allows us to lift $\left.\bar{\psi}\right|_{\operatorname{Gal}\left(\hat{K}_{0, \mathfrak{p}}\right)}$ to a homomorphism $\psi_{\mathfrak{p}}^{\prime}$ such that $\gamma \circ \psi_{\mathfrak{p}}^{\prime}=\left.\bar{\psi}\right|_{\operatorname{Gal}\left(\hat{K}_{0, \mathfrak{p}}\right)}$. The second possibility is that $\mathfrak{p} \in \bar{R}$. As mentioned in the previous paragraph, either $\left.\bar{\psi}\right|_{\operatorname{Gal}\left(\hat{K}_{0, \mathfrak{p}}\right)}$ is unramified or cyclically ramified, so we can use 
Lemma 1.2.6 or Lemma 1.2 .7 to lift $\left.\bar{\psi}\right|_{\operatorname{Gal}\left(\hat{K}_{0, \mathfrak{p}}\right)}$ to a homomorphism $\psi_{\mathfrak{p}}^{\prime}$ with $\gamma \circ \psi_{\mathfrak{p}}^{\prime}=\left.\bar{\psi}\right|_{\operatorname{Gal}\left(\hat{K}_{0, \mathfrak{p}}\right)}$. Finally, if $\mathfrak{p} \notin T \cup \bar{R}$, then $\mathfrak{p}$ is unramified in $\bar{K}$. It this case, we use Lemma 1.2 .6 to lift $\left.\bar{\psi}\right|_{\operatorname{Gal}\left(\hat{K}_{0, \mathfrak{p}}\right)}$.

THE CROSSED HOMOMORPHISM $\chi$. The construction of the crossed homomorphism $\chi$ mentioned in the preceding paragraphs with bounded ramification is a central ingredient of this work. It occupies the greater part of Chapters 2 and 3. The element $x$ of $H^{1}\left(\operatorname{Gal}\left(K_{0}\right), A\right)$ that $\chi$ represents is itself a product $x=\left(\operatorname{cor}_{\operatorname{Gal}(K)}^{\operatorname{Gal}\left(K_{0}\right)} h\right) \cdot z$, where $z$ is an element of $H^{1}\left(\operatorname{Gal}\left(K_{0}\right), H\right)$ established in Lemma 3.3.4 (due to [Neu79]) and $h: \operatorname{Gal}(K) \rightarrow A$ is a homomorphism produced by Proposition 2.4.1. Note that $\operatorname{Gal}(K)$ acts trivially on $A$, hence $h$ can be viewed as an element of $H^{1}(\operatorname{Gal}(K), A)$. Proposition 2.4.1 is one of the main ingredients of the proof of Proposition B. It constructs the homomorphism $h: \operatorname{Gal}(K) \rightarrow A$ with a bound on its ramification that leads to a bound on the ramification of $\chi$, and hence that of $\bar{\psi}$ in Proposition B. It is proved by induction over $r$ starting from Corollary 2.3.6 which is a translation of Lemma 2.3.5 by class field theory. Lemma 2.3.5 itself is modelled after Lemma 7.1 of GeJ98.

\section{OUTLINE OF THE CONTENTS OF THE CHAPTERS}

Chapter 1. Preliminaries. This chapter introduces the basic notions and tools needed in our work. First we explain what embedding problems are and what do we mean by ramification of homomorphisms. Then, we introduce the basic Galois extension $K / K_{0}$ and a basic set $S_{0}$ of primes of $K$. Each finite set $S$ of primes of $K$ that contains $S_{0}$ satisfies $C_{K}=I_{K, S} / K_{S}$, where $C_{K}$ is the idele class group of $K, I_{K, S}$ is the group of $S$-ideles, and $K_{S}$ is the group of $S$-units. The fourth and last section of the chapter reviews the notion of cohomology groups, the inflation map, and the restriction map.

Chapter 2. Bound on the ramification of homomorphisms. Strengthening a 
result of GeJ98, we construct a homomorphism $h: C_{K} \rightarrow C_{l}$ with a bound on its ramification. This bound eventually leads to a bound of the ramification of the solution of the embedding problem we solve in this work. Using class field theory, we establish an isomorphism $\operatorname{Hom}(\operatorname{Gal}(K), A) \cong \operatorname{Hom}\left(C_{K}, A\right)$, where $A$ is an abelian group and use this isomorphism to translate $h$ into a homomorphism from $\operatorname{Gal}(K)$ into $A$. Finally, in the main new result of the chapter (Proposition 2.4.1), we use induction on $r$ and construct a homomorphism from $\operatorname{Gal}(K)$ to $C_{l}^{r}$ with a bound on the ramification.

Chapter 3. Bound on the ramification of crossed homomorphisms. We construct a crossed homomorphism $\chi: \operatorname{Gal}(K) \rightarrow A$ with a bound on its ramification, where $A=C_{l}^{r}$ is a simple $\operatorname{Gal}\left(K_{0}\right)$-module. A major tool in the construction is a commutative diagram that involves restriction and corestriction maps. The main new result of this chapter is Proposition 3.3.5.

Chapter 4. Solving embedding problems with bounded ramification. This chapter contains the main results of this work. Following [Neu79], we prove in the first section that the set of all solutions of an embedding problem with an abelian kernel $A$, up to equivalence, is a principal homogeneous space over $H^{1}\left(\operatorname{Gal}\left(K_{0}\right), A\right)$. In particular, the product of a solution and a crossed homomorphism is again a solution. The second section deals with the solution of embedding problems having an abelian kernel which is a simple $\operatorname{Gal}\left(K / K_{0}\right)$-module. In the third section we carry out the induction step and conclude the proof of the main theorem. The main new results of the chapter are Proposition 4.2.2, Theorem 4.3.2, Theorem 4.4.1, and Corollary 4.4.4. 


\section{Chapter 1}

\section{Preliminaries}

This work is dominated for its large part by class field theory and cohomology theory. The aim of this chapter is to provide the necessary notions about these theories that are used in this work. Also we fix some notations and technical terms about embedding problems and ramification of homomorphisms.

\subsection{Topological Group Homomorphisms}

First, let us establish some conventions about homomorphisms of topological groups that we will assume for the rest of this work.

Let $G$ and $A$ be topological groups. Subgroups are by definition closed, so $\langle t\rangle$ is the closed subgroup generated by $t$, for $t \in G$. Every homomorphism $\varphi: G \rightarrow A$ in the category of topological groups is, by definition, continuous. Therefore, whenever we speak about a homomorphism $\varphi: G \rightarrow A$, we tacitely assume that $\varphi$ is continuous.

In the rare occasions, when $\varphi$ is constructed from a previously given con- 
tinuous homomorphisms and we only know that $\varphi\left(g_{1} g_{2}\right)=\varphi\left(g_{1}\right) \varphi\left(g_{2}\right)$ for all $g_{1}, g_{2} \in G$, then we refer to $\varphi$ as an "abstract homomorphism".

Whenever $A$ is a finite group, we consider $A$ as a topological group with the discrete topology. In this case, an abstract homomorphism $\varphi$ from a topological group $G$ to $A$ is a homomorphism if and only if $\operatorname{Ker}(\varphi)$ is open in $G$.

In particular, if $G$ is a profinite group and $A$ is finite, then an abstract homomorphism $\varphi: G \rightarrow A$ is a homomorphism if and only if $\operatorname{Ker}(\varphi)$ is an open subgroup of $G$. If, in addition, $N / K$ is a Galois extension and $G=\operatorname{Gal}(N / K)$, then $\varphi$ is a homomorphism if and only if $K$ has a finite extension $L$ in $N$ with $\varphi(\operatorname{Gal}(N / L))=1$.

Next we consider a $\mathfrak{p}$-adic field $F$ with a valuation $v$, a ring of integers $\mathcal{O}$, and a prime element $\pi$. Then, $F^{\times}=\langle\pi\rangle \times U$, where $U$ is the group of units of $\mathcal{O}$. One knows that $U$ is a profinite group under the $\mathfrak{p}$-adic topology (Proof of Proposition 5.1, p. 135 of [Neu99]). In particular, for each integer $m$, the group $\left\langle\pi^{m}\right\rangle U$ is an open subset of $F^{\times}$. Therefore, $h(U)=1$ is a sufficient condition for an abstract homomorphism $h: F^{\times} \rightarrow A$ to be a homomorphism.

\subsection{Embedding Problems and Ramification}

In this section we briefly review embedding problems and ramification of homomorphisms. We define the equivalence class of a solution, and give special cases where the local embedding problems have solutions. Recall that every given homomorphism of profinite groups is assumed to be continuous.

Let $K_{0}$ be a number field and $\mathfrak{p} \in \mathbb{P}\left(K_{0}\right)$. We write $\hat{K}_{0, \mathfrak{p}}$ for the completion of $K_{0}$ at $\mathfrak{p}$, considering $K_{0}$ as a subfield of $\hat{K}_{0, \mathfrak{p}}$. Then we embed $\tilde{K}_{0}$ into $\widehat{\hat{K}_{0, \mathfrak{p}}}$, thereby extending $\mathfrak{p}$ to $\tilde{K}_{0}$. Then $K_{0, \mathfrak{p}}=\tilde{K}_{0} \cap \hat{K}_{0, \mathfrak{p}}$ is a Henselian 
closure (resp. real closure or algebraic closure) of $K_{0}$ with respect to $\mathfrak{p}$ if $\mathfrak{p}$ is nonarchimedean (resp. real or complex). Note that $K_{0, \mathfrak{p}}$ is unique up to $K_{0}$-isomorphism. The absolute Galois group $\operatorname{Gal}\left(K_{0, \mathfrak{p}}\right)$ of $K_{0, \mathfrak{p}}$ is the absolute decomposition group $D_{\mathfrak{p}}$ of $\mathfrak{p}$. If $\mathfrak{p} \in \mathbb{P}_{\text {fin }}\left(K_{0}\right)$ then by Krasner's Lemma

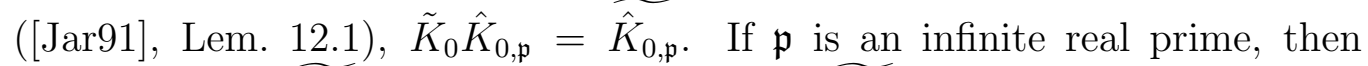
$\hat{K}_{0, \mathfrak{p}}=\mathbb{R}$ and $\widehat{\hat{K}_{0, \mathfrak{p}}}=\mathbb{C}$. Hence $\tilde{K}_{0} \hat{K}_{0, \mathfrak{p}}=\widehat{\hat{K}_{0, \mathfrak{p}}}$. If $\mathfrak{p}$ is an infinite complex prime, then $\hat{K}_{0, \mathfrak{p}}=\mathbb{C}$, so $\tilde{K}_{0} \hat{K}_{0, \mathfrak{p}}=\widehat{\hat{K}_{0, \mathfrak{p}}}$. It follows that in each case $\operatorname{res}_{\tilde{K}_{0}}: \operatorname{Gal}\left(\hat{K}_{0, \mathfrak{p}}\right) \longrightarrow \operatorname{Gal}\left(K_{0, \mathfrak{p}}\right)$ is an isomorphism. For each $\mathfrak{p} \in \mathbb{P}_{\text {fin }}\left(K_{0}\right)$, we denote $\hat{K}_{0, \mathfrak{p} \text {,ur }}$ the maximal unramified extension of $\hat{K}_{0, \mathfrak{p}}$ and $\hat{I}_{\mathfrak{p}}=\operatorname{Gal}\left(\hat{K}_{0, \mathfrak{p} \text {,ur }}\right)$ the inertia group.

Next consider a finite Galois extension $K$ of $K_{0}$ and a prime $\mathfrak{p} \in \mathbb{P}_{\text {fin }}\left(K_{0}\right)$. Choose a primitive element $x$ of $K / K_{0}$ and let $f=\operatorname{irr}\left(x, K_{0}\right)$. Then, consider the decomposition $f=f_{1} \cdots f_{m}$ of $f$ into irreducible factors over $\hat{K}_{0, \mathfrak{p}}$. For each $1 \leq i \leq m$ choose a root $x_{i}$ of $f_{i}$ in $\tilde{K}_{0}$ assuming $x_{1}=x$. Then, the map $x \mapsto x_{i}$ extends the inclusion map $K_{0} \rightarrow \hat{K}_{0, \mathfrak{p}}$ into an embedding $\lambda_{i}: K \rightarrow \hat{K}_{0, \mathfrak{p}}\left(x_{i}\right)$. In particular, $\lambda_{1}: K \rightarrow \hat{K}_{0, \mathfrak{p}}(x)$ is the inclusion map. We extend $\lambda_{i}$ to an embedding $\lambda_{i}: \tilde{K}_{0} \rightarrow \hat{K}_{0, \mathfrak{p}}$. The $\mathfrak{p}$-adic valuation $\operatorname{ord}_{\mathfrak{p}}$ of $\hat{K}_{0, \mathfrak{p}}$ uniquely extends to a discrete valuation of $\hat{K}_{0, \mathfrak{p}}\left(x_{i}\right)$ that we also denote by $\operatorname{ord}_{\mathfrak{p}}$. Pulling $\operatorname{ord}_{\mathfrak{p}}$ back to $K$ via $\lambda_{i}$ defines a prime $\mathfrak{P}_{i} \in \mathbb{P}(K)$. Specifically, $\operatorname{ord}_{\mathfrak{P}_{i}}(y)=\operatorname{ord}_{\mathfrak{p}}\left(\lambda_{i}(y)\right)$ for each $y \in K$. By [Jan73], p. 88, Thm. 5.1 or [Lan70], p. 38, Thm. 2, $\mathfrak{P}_{1}, \ldots, \mathfrak{P}_{m}$ are the distinct primes of $K$ that lie over $\mathfrak{p}$ and $\hat{K}_{\mathfrak{P}_{i}}=\hat{K}_{0, \mathfrak{p}}\left(x_{i}\right)$ is a completion of $K$ at $\mathfrak{P}_{i}$. Furthermore, $K_{\mathfrak{P}_{i}}=\left(\hat{K}_{\mathfrak{P}_{i}}\right)^{\lambda_{i}^{-1}}$ is a Henselization of $K$ at $\mathfrak{P}_{i}$ and $\operatorname{Gal}\left(K_{\mathfrak{P}_{i}}\right)^{\lambda_{i}}=\operatorname{Gal}\left(\hat{K}_{\mathfrak{P}_{i}}\right)$. Let $\hat{K}_{\mathfrak{P}_{i} \text {,ur }}$ be the maximal unramified extension of $\hat{K}_{\mathfrak{P}}$ and $\hat{I}_{\mathfrak{P}}=\operatorname{Gal}\left(\hat{K}_{\mathfrak{P}, \text { ur }}\right)$ the inertia group of $\mathfrak{P}$. Then, $K_{\mathfrak{P}_{i}, \text { ur }}=\left(\hat{K}_{\mathfrak{P}_{i}, \text { ur }}\right)^{\lambda_{\mathfrak{P}_{i}}^{-1}}$ is the maximal unramified extension of $K_{\mathfrak{P}_{i}}$ with absolute Galois group $I_{\mathfrak{P}_{i}}=\hat{I}_{\mathfrak{P}_{i}}^{\lambda_{\mathfrak{P}}}$.

If $\mathfrak{P}$ is a prime lying over $\mathfrak{p}$, then $\mathfrak{P}=\mathfrak{P}_{i}$ for some $1 \leq i \leq m$. We write $\lambda_{\mathfrak{P}}$ for $\lambda_{i}$ and $x_{\mathfrak{P}}$ for $x_{i}$. Then, $\hat{K}_{\mathfrak{P}}=\hat{K}_{0, \mathfrak{P}}\left(x_{\mathfrak{P}}\right), K_{\mathfrak{P}}=\left(\hat{K}_{\mathfrak{P}}\right)^{\lambda_{\mathfrak{P}}^{-1}}$, $\operatorname{Gal}\left(K_{\mathfrak{P}}\right)^{\lambda_{\mathfrak{P}}}=\operatorname{Gal}\left(\hat{K}_{\mathfrak{P}}\right)$, and $I_{\mathfrak{P}}^{\lambda_{\mathfrak{P}}}=\hat{I}_{\mathfrak{P}}$. 
Let $G$ be a profinite group and $\psi: \operatorname{Gal}\left(K_{0}\right) \rightarrow G$ a homomorphism. Then, the fixed field $N$ of $\operatorname{Ker}(\psi)$ in $\tilde{K}_{0}$ is a Galois extension of $K_{0}$ with $\operatorname{Gal}\left(N / K_{0}\right) \leq$ $G$. If $\psi$ is surjective then $\operatorname{Gal}\left(N / K_{0}\right) \cong G$.

Definition 1.2.1. For each homomorphism $\psi: \operatorname{Gal}\left(K_{0}\right) \longrightarrow G$ and $\mathfrak{p} \in$ $\mathbb{P}\left(K_{0}\right)$, we define the homomorphism $\psi_{\mathfrak{p}}: \operatorname{Gal}\left(\hat{K}_{0, \mathfrak{p}}\right) \longrightarrow G$ such that $\psi_{\mathfrak{p}}(\sigma)=$ $\psi\left(\left.\sigma\right|_{\tilde{K}_{0}}\right)$

Definition 1.2.2 (Embedding Problem). Let $K / K_{0}$ be a Galois extension of number fields, $G$ a profinite group, and $\alpha: G \longrightarrow \operatorname{Gal}\left(K / K_{0}\right)$ an epimorphism. The embedding problem associated with $\alpha$ consists of embedding $K$ into a Galois extension $N$ of $K_{0}$ with an isomorphism $\beta: \operatorname{Gal}\left(N / K_{0}\right) \longrightarrow G$ satisfying $\alpha \circ \beta=\operatorname{res}_{N / K}$. It is equivalent to finding a continuous epimor$\operatorname{phism} \psi: \operatorname{Gal}\left(K_{0}\right) \longrightarrow G$ such that, if $\rho=\operatorname{res}_{\widetilde{K}_{0} / K}$, then $\alpha \circ \psi=\rho$.

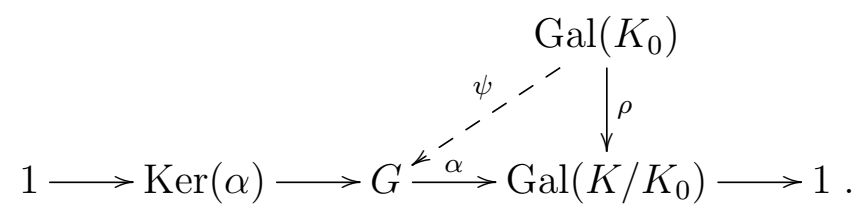

A homomorphism $\psi$ that makes the diagram 1.2.1 commute is a solution and the fixed field $N$ of $\operatorname{Ker}(\psi)$ is a solution field of the embedding problem (we also say $\psi$ is a $\operatorname{Gal}\left(K / K_{0}\right)$-homomorphism). The homomorphism $\psi$ is a proper solution if it is surjective .

For each $\mathfrak{p} \in \mathbb{P}\left(K_{0}\right)$, the global embedding problem 11.2 .1 gives rise to a local embedding problem

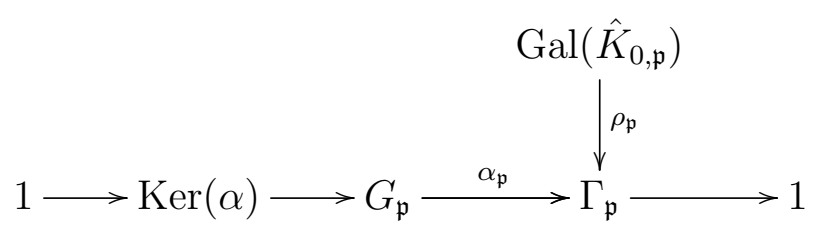

where $\rho_{\mathfrak{p}}(\sigma)=\rho\left(\left.\sigma\right|_{\tilde{K}_{0}}\right)$ for each $\sigma \in \operatorname{Gal}\left(\hat{K}_{0, \mathfrak{p}}\right), \Gamma_{\mathfrak{p}}=\rho_{\mathfrak{p}}\left(\operatorname{Gal}\left(\hat{K}_{0, \mathfrak{p}}\right)\right), G_{\mathfrak{p}}=$ $\alpha^{-1}\left(\Gamma_{\mathfrak{p}}\right), \alpha_{\mathfrak{p}}=\left.\alpha\right|_{G_{\mathfrak{p}}}$, and $\operatorname{Ker}(\alpha)=\operatorname{Ker}\left(\alpha_{\mathfrak{p}}\right)$. If $\psi$ is a solution of embedding problem (1.2.1) then $\psi_{\mathfrak{p}}$ (Def. 1.2.1) is a solution of the local embedding 
problem 1.2 .2 .

The solutions of an embedding problem form equivalence classes as follows.

Definition 1.2.3 (Conjugate Homomorphisms). Let $\psi_{1}: \operatorname{Gal}\left(K_{0}\right) \longrightarrow G$ and $\psi_{2}: \operatorname{Gal}\left(K_{0}\right) \longrightarrow G$ be homomorphisms that make 1.2 .1$)$ commute, that is $\alpha \circ \psi_{1}=\alpha \circ \psi_{2}=\rho$. We say that $\psi_{1}$ and $\psi_{2}$ are $\operatorname{Ker}(\alpha)$-conjugate if there exists an element $a \in \operatorname{Ker}(\alpha)$ such that

$$
\psi_{2}(\sigma)=a^{-1} \psi_{1}(\sigma) a \text { for all } \sigma \in \operatorname{Gal}\left(K_{0}\right)
$$

This is an equivalence relation on the set of the solutions of 1.2 .1 . We denote by $\left[\psi_{1}\right]$ the equivalence class of $\psi_{1}$. Let $\Gamma=\operatorname{Gal}\left(K / K_{0}\right)$. We denote by $\mathcal{H} \operatorname{om}_{\Gamma, \rho, \alpha}\left(\operatorname{Gal}\left(K_{0}\right), G\right)$ the set of all equivalence classes of homomorphisms which make 1.2.1 commute (or $\Gamma$-homomorphisms).

Note that if $\psi_{1}$ and $\psi_{2}$ are $\operatorname{Ker}(\alpha)$-conjugate, then $\operatorname{Ker}\left(\psi_{1}\right)=\operatorname{Ker}\left(\psi_{2}\right)$. Thus, every element of an equivalence class $[\psi]$ yields the same solution field of the embedding problem. Also, if $\psi$ is surjective, then so is every element of $[\psi]$. We denote by $\mathcal{H o m}_{\Gamma, \rho, \alpha}\left(\operatorname{Gal}\left(K_{0}\right), G\right)_{\text {sur }}$ the subset of all equivalence classes of epimorphisms.

Similarly, let $\psi_{1, \mathfrak{p}}: \operatorname{Gal}\left(\hat{K}_{0, \mathfrak{p}}\right) \longrightarrow G$ and $\psi_{2, \mathfrak{p}}: \operatorname{Gal}\left(\hat{K}_{0, \mathfrak{p}}\right) \longrightarrow G$ be homomorphisms which make 1.2 .2 commute $\left(\Gamma_{\mathfrak{p}}\right.$-homomorphisms). Then $\psi_{1, \mathfrak{p}}$ and $\psi_{2, \mathfrak{p}}$ are $\operatorname{Ker}\left(\alpha_{\mathfrak{p}}\right)$-conjugate if there exists an element $a \in \operatorname{Ker}\left(\alpha_{\mathfrak{p}}\right)$ such that

$$
\psi_{2, \mathfrak{p}}(\sigma)=a^{-1} \psi_{1, \mathfrak{p}}(\sigma) a \text { for all } \sigma \in \operatorname{Gal}\left(K_{0, \mathfrak{p}}\right)
$$

Note that $\operatorname{Ker}(\alpha)=\operatorname{Ker}\left(\alpha_{\mathfrak{p}}\right)$. To simplify the notation, we only say that $\psi_{1, \mathfrak{p}}$ and $\psi_{2, \mathfrak{p}}$ are $\operatorname{Ker}(\alpha)$-conjugate. We denote by $\mathcal{H} \mathrm{om}_{\Gamma, \rho_{\mathfrak{p}}, \alpha_{\mathfrak{p}}}\left(\operatorname{Gal}\left(\hat{K}_{0, \mathfrak{p}}\right), G\right)$ the set of all equivalence classes $\left[\psi_{\mathfrak{p}}\right]$.

Note that if $\psi_{1}: \operatorname{Gal}\left(K_{0}\right) \longrightarrow G$ and $\psi_{2}: \operatorname{Gal}\left(K_{0}\right) \longrightarrow G$ are $\operatorname{Ker}(\alpha)$-conjugate, then so are $\psi_{1, \mathfrak{p}}$ and $\psi_{2, \mathfrak{p}}$ (Def. 1.2.1), for each $\mathfrak{p} \in \mathbb{P}\left(K_{0}\right)$. Hence, we have 
the canonical map

$$
\begin{aligned}
\mathcal{H}_{\Gamma, \rho, \alpha}\left(\operatorname{Gal}\left(K_{0}\right), G\right) & \longrightarrow \prod_{\mathfrak{p}} \mathcal{H} \operatorname{Hom}_{\Gamma, \rho_{\mathfrak{p}}, \alpha_{\mathfrak{p}}}\left(\operatorname{Gal}\left(K_{0, \mathfrak{p}}\right), G\right) \\
{[\psi] } & \longmapsto\left(\left[\psi_{\mathfrak{p}}\right]\right)_{\mathfrak{p}}
\end{aligned}
$$

Now let us define some properties of a homomorphism.

Definition 1.2.4. Let $\psi: \operatorname{Gal}\left(K_{0}\right) \rightarrow G$ be a homomorphism and let $L$ be the fixed field of $\operatorname{Ker}(\psi)$ in $\tilde{K}_{0}$. Let $\mathfrak{p} \in \mathbb{P}_{\text {fin }}\left(K_{0}\right)$. We say that

(a) $\psi$ totally decomposes at $\mathfrak{p}$ if $\psi_{\mathfrak{p}}\left(\operatorname{Gal}\left(\hat{K}_{0, \mathfrak{p}}\right)=1\right.$ (i.e. $\psi\left(K_{0, \mathfrak{p}}\right)=1$ ), that is $L \subseteq K_{0, \mathfrak{p}}$. In this case $\mathfrak{p}$ totally splits in $L$. In the case where $L / K_{0}$ is finite, then $L$ has $\left[L: K_{0}\right]$ primes that lie over $\mathfrak{p}$.

(b) $\psi$ is unramified at $\mathfrak{p}$ if $\mathfrak{p}$ is unramified in $L$, that is $\psi_{\mathfrak{p}}\left(\hat{I}_{\mathfrak{p}}\right)=1$. We denote the set of primes at which $\psi$ ramifies by $\operatorname{Ram}(\psi)$. Then $\operatorname{Ram}(\psi)=\operatorname{Ram}\left(L / K_{0}\right)$.

(c) $\psi$ is cyclic if it factors through a cyclic extension of $K_{0}$, that is, there exists a cyclic extension $K^{\prime} / K_{0}$ such that $\psi\left(\operatorname{Gal}\left(K^{\prime}\right)\right)=1$.

Remark 1.2.5. Note that if a $\Gamma$-homomorphism $\psi: \operatorname{Gal}\left(K_{0}\right) \longrightarrow G$ is unramified at a prime $\mathfrak{p}$ then each $\Gamma$-homomorphism in the equivalence class $[\psi] \in \mathcal{H}_{\Gamma, \rho, \alpha}\left(\operatorname{Gal}\left(K_{0}\right), G\right)$ is also unramified at $\mathfrak{p}$ since $\operatorname{Ker}\left(\psi^{\prime}\right)=\operatorname{Ker}(\psi)$ for each $\psi^{\prime} \in[\psi]$. In this case we say that $[\psi]$ is unramified at $\mathfrak{p}$.

Special local embedding problems. In view of the canonical map (1.2.5, a necessary condition for the global embedding problem (1.2.1) to have a solution is that each corresponding local embedding problem 1.2 .2 has a solution.

The following lemma ensures that the local embedding problem 1.2 .2 induced by the global embedding problem 1.2.1) has a solution if $\mathfrak{p}$ is unramified in $K$. 
Lemma 1.2.6. Let $\lambda: G \longrightarrow \bar{G}$ be an epimorphism of profinite groups. Let $\mathfrak{p} \in \mathbb{P}\left(K_{0}\right)$, and let $\bar{\psi}_{\mathfrak{p}}: \operatorname{Gal}\left(\hat{K}_{0, \mathfrak{p}}\right) \longrightarrow \bar{G}$ be an unramified homomorphism. Then, $\bar{\psi}_{\mathfrak{p}}$ can be lifted to an unramified $\bar{G}$-homomorphism $\psi_{\mathfrak{p}}: \operatorname{Gal}\left(\hat{K}_{0, \mathfrak{p}}\right) \longrightarrow$ G. Thus $\mathcal{H}_{\mathrm{om}_{\bar{G}, \psi_{\mathfrak{p}}, \lambda}}\left(\operatorname{Gal}\left(\hat{K}_{0, \mathfrak{p}}\right), G\right) \neq \emptyset$.

Proof. Since $\bar{\psi}_{\mathfrak{p}}$ is unramified, there exists a unique homomorphism $\bar{\varphi}_{\mathfrak{p}}: \operatorname{Gal}\left(\hat{K}_{0, \mathfrak{p}}\right) / \hat{I}_{\mathfrak{p}} \longrightarrow \bar{G}$ such that $\bar{\varphi}_{\mathfrak{p}} \circ \pi=\bar{\psi}_{\mathfrak{p}}$, where $\pi: \operatorname{Gal}\left(\hat{K}_{0, \mathfrak{p}}\right) \longrightarrow$ $\operatorname{Gal}\left(\hat{K}_{0, \mathfrak{p}}\right) / \hat{I}_{\mathfrak{p}}$ is the quotient map. One knows that $\operatorname{Gal}\left(\hat{K}_{0, \mathfrak{p}}\right) / \hat{I}_{\mathfrak{p}} \cong \hat{\mathbb{Z}}([\operatorname{Se} 79]$, p. 55). Let $\bar{\sigma}$ be the image of $1 \in \hat{\mathbb{Z}}$ under this isomorphism. Let $\bar{g} \in \bar{G}$ such that $\bar{g}=\bar{\varphi}_{\mathfrak{p}}(\bar{\sigma})$, and let $g \in G$ such that $\lambda(g)=\bar{g}$. Define a continuous homomorphism $\bar{\varphi}_{\mathfrak{p}}^{\prime}: \operatorname{Gal}\left(\hat{K}_{0, \mathfrak{p}}\right) / \hat{I}_{\mathfrak{p}} \longrightarrow G$ by $\bar{\varphi}_{\mathfrak{p}}^{\prime}(\bar{\sigma})=g$. Then $\lambda \circ \bar{\varphi}_{\mathfrak{p}}^{\prime}=\bar{\varphi}_{\mathfrak{p}}$. Consider the continuous homomorphism $\psi_{\mathfrak{p}}: \operatorname{Gal}\left(\hat{K}_{0, \mathfrak{p}}\right) \stackrel{\pi}{\longrightarrow} \operatorname{Gal}\left(\hat{K}_{0, \mathfrak{p}}\right) / \hat{I}_{\mathfrak{p}} \stackrel{\bar{\varphi}_{\mathfrak{p}}^{\prime}}{\longrightarrow} G$. We have, $\lambda \circ \psi_{\mathfrak{p}}=\lambda \circ \bar{\varphi}_{\mathfrak{p}}^{\prime} \circ \pi=\bar{\varphi}_{\mathfrak{p}} \circ \pi=\bar{\psi}_{\mathfrak{p}}$. Thus, $\psi_{\mathfrak{p}}$ is a $\bar{G}$-homomorphism, i.e. $\left[\psi_{\mathfrak{p}}\right] \in \mathcal{H}^{\circ} \mathrm{m}_{\bar{G}, \bar{\psi}_{\mathfrak{p}, \lambda}}\left(\operatorname{Gal}\left(\hat{K}_{0, \mathfrak{p}}\right), G\right)$.

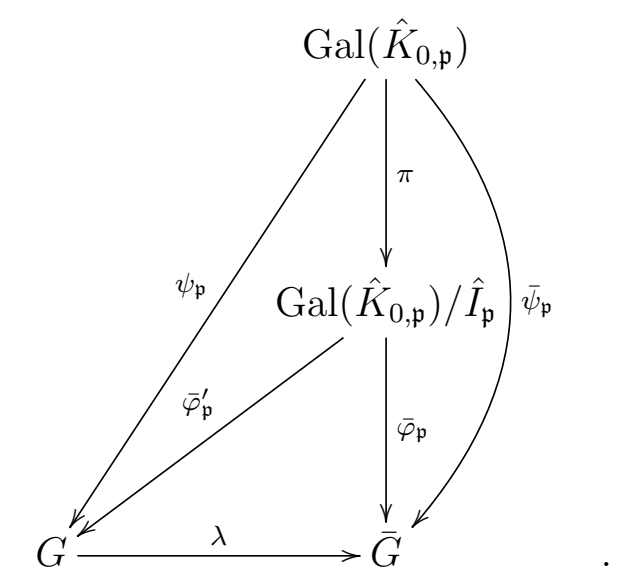

Moreover, for each $\tau \in \hat{I}_{\mathfrak{p}}$, we have $\psi_{\mathfrak{p}}(\tau)=\bar{\varphi}_{\mathfrak{p}}^{\prime}(\pi(\sigma))=\bar{\varphi}_{\mathfrak{p}}^{\prime}(1)=1$. Thus $\psi_{\mathfrak{p}}$ is unramified.

If the given homomorphism ramifies at the prime $\mathfrak{p}$, under some other conditions, the case where it is locally cyclic at $\mathfrak{p}$ allows us to solve the corresponding local embedding problem.

Lemma 1.2.7. Let $\lambda: G \rightarrow \bar{G}$ be an epimorphism of profinite groups with finite kernel. Suppose $C_{l} \leq \bar{G}$. Set $e=|\operatorname{Ker}(\lambda)|$ and let $n$ be a multiple 
of $e l$. Let $\mathfrak{p} \in \mathbb{P}\left(K_{0}\right)$ with $\mathfrak{p} \nmid l$. Suppose $\zeta_{n} \in K_{0, \mathfrak{p}}$. If $\bar{\psi}_{\mathfrak{p}}: \operatorname{Gal}\left(K_{0, \mathfrak{p}}\right) \rightarrow$ $C_{l} \leq \bar{G}$ is ramified $\left(\bar{\psi}_{\mathfrak{p}}\left(I_{\mathfrak{p}}\right) \neq 1\right)$, then $\bar{\psi}_{\mathfrak{p}}$ can be lifted to a $\bar{G}$-homomorphism $\psi_{\mathfrak{p}}: \operatorname{Gal}\left(K_{0, \mathfrak{p}}\right) \rightarrow G$.

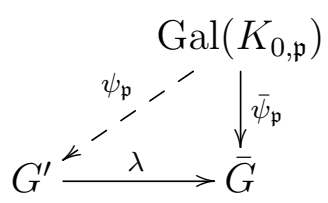

Proof. Let $N_{\mathfrak{p}}$ be the fixed field of $\operatorname{Ker}\left(\bar{\psi}_{\mathfrak{p}}\right)$. Then $N_{\mathfrak{p}} / \hat{K}_{0, \mathfrak{p}}$ is a cyclic ramified extension of degree $l$. Since $\mathfrak{p} \nmid l$, the ramification is tame. By Proposition 1(i) of ([CaF67], p. 32), there exists a prime element $\pi$ of $\hat{K}_{0, \mathfrak{p}}$ with $N_{\mathfrak{p}}=\hat{K}_{0, \mathfrak{p}}(\sqrt[l]{\pi})$ Let $\bar{\sigma}$ be a generator of $\operatorname{Gal}\left(N_{\mathfrak{p}} / \hat{K}_{0, \mathfrak{p}}\right)$ and choose $\sigma \in G$ with $\lambda(\sigma)=\bar{\sigma}$.

Denote the order of $\sigma$ by $d$, let $\lambda^{\prime}=\left.\lambda\right|_{\langle\sigma\rangle}$, and set $e^{\prime}=\left|\operatorname{Ker}\left(\lambda^{\prime}\right)\right|$. Since $\operatorname{Ker}\left(\lambda^{\prime}\right)$ is a subgroup of $\operatorname{Ker}(\lambda)$, we have $e^{\prime} \mid e$. Since $d=e^{\prime} l, e^{\prime} \mid e$ and $e l \mid n$, then $d \mid n$. Hence, $\zeta_{d} \in \hat{K}_{0, \mathfrak{p}}$. Thus, $N_{\mathfrak{p}}^{\prime}=\hat{K}_{0, \mathfrak{p}}(\sqrt[d]{\pi})$ is a cyclic extension of $\hat{K}_{0, \mathfrak{p}}$ of degree $d$ that contains $N_{\mathfrak{p}}$. Since $N_{\mathfrak{p}}$ is the fixed field of $\operatorname{Ker}\left(\bar{\psi}_{\mathfrak{p}}\right)$, there exists an epimorphism $\bar{\varphi}_{\mathfrak{p}}: \operatorname{Gal}\left(N_{\mathfrak{p}}^{\prime} / \hat{K}_{0, \mathfrak{p}}\right) \longrightarrow \operatorname{Gal}\left(N_{\mathfrak{p}} / \hat{K}_{0, \mathfrak{p}}\right)$ such that $\bar{\psi}_{\mathfrak{p}}=\bar{\varphi}_{\mathfrak{p}} \circ \operatorname{res} \underset{\widetilde{K_{0, \mathfrak{p}}} / N_{\mathfrak{p}}^{\prime}}{ }$.

We choose a generator $\tau$ of $\operatorname{Gal}\left(N_{\mathfrak{p}}^{\prime} / \hat{K}_{0, \mathfrak{p}}\right)$ such that $\bar{\varphi}_{\mathfrak{p}}(\tau)=\bar{\sigma}$. Consider the homomorphism $h: \operatorname{Gal}\left(N_{\mathfrak{p}}^{\prime} / \hat{K}_{0, \mathfrak{p}}\right) \longrightarrow G$ with $h(\tau)=\sigma$. Then, the homomorphism $\psi_{\mathfrak{p}}=h \circ \operatorname{res} \underset{\widehat{K_{0, \mathfrak{p}}} / N_{\mathfrak{p}}^{\prime}}{ }$ satisfies $\lambda \circ \psi_{\mathfrak{p}}=\bar{\psi}_{\mathfrak{p}}$.

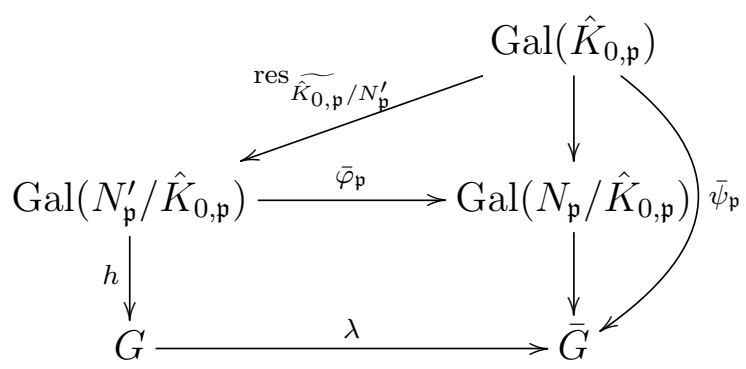

The condition that each local embedding problem has a solution is not sufficient for the global embedding problem to have a solution. However, under 
some additional conditions, we have the following local-global principle.

Lemma 1.2.8 ([Neu79], Lem. 4). Consider the embedding problem (1.2.1). Suppose $\operatorname{Ker}(\alpha) \cong C_{l}^{r}$, for some prime number $l$, is a simple $\operatorname{Gal}\left(K_{0}\right)$-module (with the action induced by the homomorphism $\alpha$ and then by $\rho$ ). If $\zeta_{l} \notin K$, then

$$
\mathcal{H} \operatorname{om}_{\Gamma, \rho, \alpha}\left(\operatorname{Gal}\left(K_{0}\right), G\right) \neq \emptyset \text { if and only if } \prod_{\mathfrak{p}} \mathcal{H} \mathrm{om}_{\Gamma, \rho_{\mathfrak{p}}, \alpha}\left(\operatorname{Gal}\left(\hat{K}_{0, \mathfrak{p}}\right), G\right) \neq \emptyset \text {. }
$$

One of the important tools in bounding the ramification of a solution of an embedding problem is the Chebotarev density theorem.

Chebotarev Density Theorem. Let $L / K_{0}$ be a finite Galois extension of Global fields and let $\mathcal{C}$ be a conjugacy class in $\operatorname{Gal}\left(L / K_{0}\right)$. Then the Dirichlet density of $\left\{\mathfrak{p} \in \mathbb{P}\left(K_{0}\right) \mid\left(\frac{L / K_{0}}{\mathfrak{p}}\right)=\mathcal{C}\right\}$ exists and is equal to $\frac{|\mathcal{C}|}{\left[L: K_{0}\right]}$.

The Artin symbol $\left(\frac{L / K_{0}}{\cdot}\right)$ is defined only for the primes $\mathfrak{p} \in \mathbb{P}\left(K_{0}\right)$ which are unramified in $L$, so the prime chosen in accordance with the Chebotarev density theorem is consequently unramified ([FrJ08], Thm. 6.3.1).

Note that a prime $\mathfrak{p}$ of $K_{0}$ totally splits in $L$ (i.e $L$ has $\left[L: K_{0}\right]$ primes that lie over $\mathfrak{p})$ if and only if the Frobenius $\left[\frac{L / K_{0}}{\mathfrak{P}}\right]$ is equal to 1 , for $\mathfrak{P} \in \mathbb{P}(L)$ with $\mathfrak{P} \mid \mathfrak{p}$. Thus, applying the Chebotarev density theorem in the case where $\mathcal{C}=1$, we conclude that $K_{0}$ has infinitely many primes that totally split in $L$.

\subsection{A Basic Set of Primes and the Reciprocity Law}

The reciprocity law plays a significant role in the construction of a homomorphism with a bound on its ramification. In this section, we present results from class field theory which we apply in this work. For details, we refer the 
reader to chapters 4, 5 and 6 of [Neu99], or to chapters 6 and 7 of [CaF67].

Let $K$ be a number field and let $\mathfrak{P} \in \mathbb{P}(K)$. If $\mathfrak{P}$ is archimedean, then $\hat{K}_{\mathfrak{P}}=\mathbb{R}$ or $\hat{K}_{\mathfrak{P}}=\mathbb{C}$. In this case, set $U_{\mathfrak{P}}=\hat{K}_{\mathfrak{P}}^{\times}$and $\pi_{\mathfrak{P}}=1$. If $\mathfrak{P}$ is nonarchimedean, then $\hat{K}_{\mathfrak{P}}$ is a complete discrete valuation field with a finite residue field. In this case, we denote the corresponding normalized valuation by $v_{\mathfrak{P}}$, and we choose a prime element $\pi_{\mathfrak{P}}$ in $\hat{K}_{\mathfrak{P}}$, that is $v_{\mathfrak{P}}\left(\pi_{\mathfrak{P}}\right)=1$. We set $U_{\mathfrak{P}}$ to be the group of units of $\hat{K}_{\mathfrak{P}}$.

For a finite set $S$ of primes of $K$ such that $\mathbb{P}_{\infty}(K) \subseteq S$, we define the group of $S$-units of $K$ as $K_{S}=\left\{x \in K \mid v_{\mathfrak{P}}(x)=0\right.$ for all $\left.\mathfrak{P} \notin S\right\}$.

An idele of $K$ is an element $\alpha=\left(\alpha_{\mathfrak{P}}\right)_{\mathfrak{P}} \in \prod_{\mathfrak{P} \in \mathbb{P}(K)} \hat{K}_{\mathfrak{P}}^{\times}$, where $\alpha_{\mathfrak{P}} \in U_{\mathfrak{P}}$ for all but finitely many $\mathfrak{P}$. The ideles of $K$ form a multiplicative group denoted by $I_{K}$. That is, $I_{K}$ is the restricted product of the multiplicative groups $\hat{K}_{\mathfrak{P}}$ with respect to the subgroups $U_{\mathfrak{P}}$. A basis of neighbourhoods of $1 \in I_{K}$ is given by the sets

$$
\prod_{\mathfrak{P} \in S} V_{\mathfrak{P}} \times \prod_{\mathfrak{P} \notin S} U_{\mathfrak{P}}
$$

where $S$ runs over the finite sets of primes of $K$ with $\mathbb{P}_{\infty}(K) \subseteq S$ and $V_{\mathfrak{P}}$ runs over a basis of neighbourhoods of $1 \in \hat{K}_{\mathfrak{P}}^{\times}$. In particular

$$
U_{K}=\prod_{\mathfrak{P} \in \mathbb{P}_{K}} U_{\mathfrak{P}}
$$

is open in $I_{K}$. The group $I_{K}$ is a locally compact topological group ([Neu99], p. 361).

(1.3.1) If $A$ is a finite group, then an abstract homomorphism $h: I_{K} \rightarrow A$ is continuous if and only if $h\left(U_{\mathfrak{P}}\right)=1$ for almost all $\mathfrak{P} \in \mathbb{P}(K)$ and $\left.h\right|_{\hat{K}_{\mathfrak{P}}^{\times}}$is continuous for the rest of the $\mathfrak{P}$ 's.

For a finite set $S$ of primes of $K$, the group $I_{K, S}=\prod_{\mathfrak{P} \in S} \hat{K}_{\mathfrak{P}}^{\times} \times \prod_{\mathfrak{P} \notin S} U_{\mathfrak{P}}$ is the group of $S$-ideles of $K$. 
The multiplicative group $K^{\times}$is embedded diagonally in $I_{K}$. Each $x \in K^{\times}$ corresponds under this embedding to the idele $\left(x_{\mathfrak{P}}\right)_{\mathfrak{P}}$ with $x_{\mathfrak{P}}=x$ for each $\mathfrak{P} \in \mathbb{P}(K)$. We view $K^{\times}$as a subgroup of $I_{K}$ and call its elements principal ideles. The subgroup $K^{\times}$is discrete and therefore closed in $I_{K}$ (Chapter 5 , Section 1 of [Neu99]).

The factor group $C_{K}=I_{K} / K^{\times}$is called the idele class group of $K$. It is a Hausdorff locally compact group ([Neu99], p. 361).

Let $J_{K}$ and $P_{K}$ be the group of fractional ideals and principal fractional ideals of $K$ respectively. The class group $\mathrm{Cl}_{K}=J_{K} / P_{K}$ is a finite group ([Neu99], p. 36, Thm. 6.3). Its order $h_{K}$ is the class number of $K$. The connection between idele and ideal classes of $K$ is given by $\mathrm{Cl}_{K} \cong I_{K} / U_{K} K^{\times}$ (see p. 360, Prop. 1.3 of [Neu99]). This connection implies that each element of $C_{K}$ can be represented by an $S$-idele for some finite set $S$ of primes in the following way.

Setup 1.3.1. (Basic Set) Let $\alpha_{1}, \ldots, \alpha_{h_{K}}$ be representatives of $I_{K}$ modulo $U_{K} K^{\times}$. Let $S_{0}$ be the set of infinite primes and all finite primes $\mathfrak{P}$ such that $v_{\mathfrak{P}}\left(\alpha_{i, \mathfrak{P}}\right) \neq 0$ for at least one $i$. Let $S$ be a finite set of primes with $S_{0} \subseteq S$. Then $I_{K}=I_{K, S} K^{\times}$. Indeed, for an idele $\alpha \in I_{K}$, there exists $i \in\left\{1, \ldots, h_{K}\right\}$ such that $\alpha \in \alpha_{i} U_{K} K^{\times}$. Thus $\alpha=\alpha_{i} u x$ for some $u \in U_{K}$ and $x \in K^{\times}$. Since $S_{0} \subseteq S$, for each $\mathfrak{P} \in \mathbb{P}(K) \backslash S, v_{\mathfrak{P}}\left(\alpha_{i, \mathfrak{P}}\right)=0$. Hence, $\alpha_{i} \in I_{K, S}$, so $\alpha_{i} u \in I_{K, S}$. It follows that $\alpha \in I_{K, S} K^{\times}$. By definition, $I_{K, S} \cap K^{\times}=K_{S}$. Therefore,

$$
C_{K}=I_{K, S} / K_{S}
$$

See also p. 360, Prop. 1.4 of [Neu99].

Definition. (Basic set) We add the primes of $K$ that divide a fixed prime number $l$ to the set $S_{0}$ defined in the preceding paragraph and call it a basic set of $K$. 
The following well known Lemma is useful for determining a quotient of the idele class group $C_{K}$.

Lemma 1.3.2. Let $a$ be an element of $K^{\times}$. We assume that $a$ is an $l$-power in $\hat{K}_{\mathfrak{P}}$ for every prime $\mathfrak{P} \in \mathbb{P}(K)$. Then, $a$ is an $l$-power in $K$.

Proof. Assume toward contradiction that $a$ is not an $l$-power in $K$. Then, $X^{l}-a$ is irreducible over $K$ ([Lan93], p. 297, Thm. 9.1). We denote the splitting field of $X^{l}-a$ over $K$ by $N$ and choose a root $x$ of $X^{l}-a$. Then, $H=\operatorname{Gal}(N / K(x))$ is a proper subgroup of $G=\operatorname{Gal}(N / K)$. Hence, $G \backslash$ $\bigcup_{\sigma \in G} H^{\sigma}$ is a proper subset of $G$ (Lem. 13.3.2, [FrJ08]). By Chebotarev density theorem, $K$ has a prime divisor $\mathfrak{P}$ which is unramified in $N$ such that $\left(\frac{N / K}{\mathfrak{P}}\right) \subseteq G \backslash \bigcup_{\sigma \in G} H^{\sigma}$. This implies that $X^{l}-a$ has no root in $\hat{K}_{\mathfrak{P}}$, which is a contradiction.

Remark 1.3.3. Let $S$ be a finite set of primes of $K$ with $\mathbb{P}_{\infty}(K) \subseteq S$ and $l$ a prime number. By Lemma 1.3.2, $K_{S} \cap I_{K, S}^{l}=K_{S}^{l}$. We use a bar to denote the reduction of elements and subgroups of $I_{K, S}$ modulo $I_{K, S}^{l}$. Hence,

$$
\overline{K_{S}}=K_{S} / K_{S}^{l} \cong K_{S} I_{K, S}^{l} / I_{K, S}^{l}
$$

Therefore,

$$
\overline{I_{K, S}} / \overline{K_{S}} \cong\left(I_{K, S} / I_{K, S}^{l}\right) /\left(K_{S} I_{K, S}^{l} / I_{K, S}^{l}\right) \cong I_{K, S} / K_{S} I_{K, S}^{l}
$$

is a quotient of $C_{K}=I_{K, S} / K_{S}$ (see equality 1.3.2).

Now, let $L / K$ be a finite Galois extension. A basic tool for our work is the Artin reciprocity law:

Proposition 1.3.4 (Global reciprocity law). ([Neu99],p. 391, Thm. 5.5 or [CaF67], p. 172, Thm. 5.1 (B)) For every finite Galois extension $L / K$ of number fields, we have a canonical isomorphism

$$
r_{L / K}: \operatorname{Gal}(L / K)^{\mathrm{ab}} \longrightarrow C_{K} / N_{L / K} C_{L}
$$


where $\operatorname{Gal}(L / K)^{\mathrm{ab}}$ is the maximal abelian factor group of $\operatorname{Gal}(L / K)$. The inverse of $r_{L / K}$ gives the continuous surjective norm residue symbol

$$
(, L / K): C_{K} \longrightarrow \operatorname{Gal}(L / K)^{\mathrm{ab}}
$$

with kernel $N_{L / K} C_{L}$ (see the definition of $N_{L / K}: C_{L} \longrightarrow C_{K}$ in [Neu99, p. 373).

Proposition 1.3.5 (Existence Theorem). ([Neu99], p. 395, Thm. 6.1 or CaF67, p. 172, Thm. 5.1(D)) The map $L \mapsto N_{L / K} C_{L}$ is a 1-1 correspondence between the finite abelian extensions $L / K$ and the closed subgroups of finite index in $C_{K}$.

Now, suppose $L / K$ is a finite Galois extension of nonarchimedean local fields. Similarly, we have the local reciprocity law.

Proposition 1.3.6 (Local reciprocity law). ([Neu99], p. 320, Thm. 1.3)

(a) For every finite Galois extension $L / K$ of nonarchimedean local fields, we have an isomorphism

$$
r_{L / K}: \operatorname{Gal}(L / K)^{\mathrm{ab}} \longrightarrow K^{\times} / N_{L / K} L^{\times}
$$

which gives the continuous surjective norm residue symbol

$$
(, L / K): K^{\times} \longrightarrow \operatorname{Gal}(L / K)^{\mathrm{ab}}
$$

with kernel $N_{L / K} L^{\times}$.

(b) If $L / K$ is abelian, then $(, L / K)$ maps the group of units $U_{K}$ of $K$ onto the inertia group $I(L / K)$ (p. 354, Thm. 6.2, [Neu99]).

The reciprocity law is compatible with the restriction map of finite Galois extensions:

Proposition 1.3.7. ( Neu99], p. 302, Prop. 6.4) Let $K \subseteq L \subseteq L^{\prime}$ be a tower of finite Galois extension of number fields (resp. of nonarchimedena 
local fields). Then we have the commutative diagram:

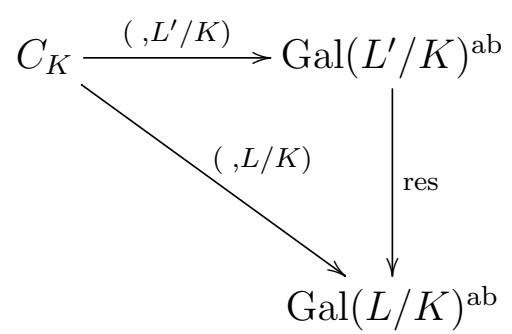

(resp.

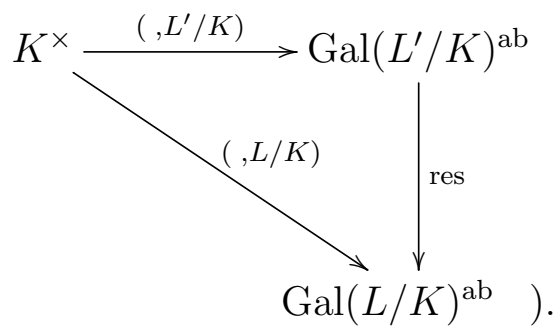

We have an embedding of $\hat{K}_{\mathfrak{P}}^{\times}$into $I_{K}$ which associates each element $\alpha \in \hat{K}_{\mathfrak{P}}^{\times}$ to $\left(\alpha_{\mathfrak{Q}}\right)_{\mathfrak{Q}} \in I_{K}$ with $\alpha_{\mathfrak{P}}=\alpha$ and $\alpha_{\mathfrak{Q}}=1$ for $\mathfrak{Q} \neq \mathfrak{P}$. Under this embedding, $\hat{K}_{\mathfrak{P}}^{\times} \cap K^{\times}=1$, so we can consider $\hat{K}_{\mathfrak{P}}^{\times}$as a subgroup of $C_{K}$. Then we have the compatibility of the local and the global reciprocity law.

Proposition 1.3.8. ([Neu99], p. 391, Prop. 5.6) Let $L / K$ be a finite abelian extension of number fields and $\mathfrak{P} \in \mathbb{P}_{\text {fin }}(K)$. Then, the following diagram commutes

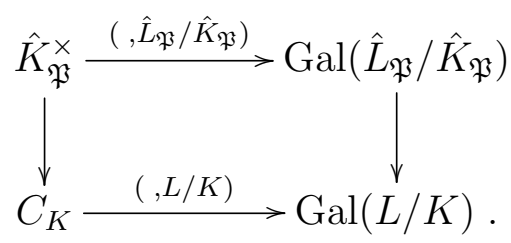

\subsection{Cohomology Groups and Special Mappings}

The modules we use in this work are multiplicative and we use exponential notation from the right for the action of the groups on the modules. In contrast, books about cohomology (e.g. [NSW00] mostly consider additive 
modules on which groups act from the left. This difference does not change the essence of the cohomology theory, because the category of left modules is equivalent to the category of right modules. However, the explicit formulas of cochains do change when we go from action from the left to action from the right.

In this section we briefly introduce the basic concepts of cohomology of modules with right action, and some special mappings. Our introduction follows Chapter I of [NSW00.

Let $G$ be a profinite group and $A$ a multiplicative abelian group on which $G$ acts from the right. The image of the action of an element $\sigma$ of $G$ on an element $a$ of $A$ is written as $a^{\sigma}$.

(A) Continuous Maps ([NSW00], p. 10,11). For each integer $n \geq 0$ the multiplicative group $X^{(n)}=\operatorname{Map}_{\text {cont }}\left(G^{n+1}, A\right)$ of all continuous maps $x: G^{n+1} \longrightarrow A$, with $A$ carrying the discrete topology, becomes a right $G$ module by setting

$$
x^{\tau}\left(\sigma_{0}, \ldots, \sigma_{n}\right)=x\left(\sigma_{0} \tau^{-1}, \ldots, \sigma_{n} \tau^{-1}\right)^{\tau} \text { for } x \in X^{(n)} \text { and } \tau, \sigma_{0}, \ldots, \sigma_{n} \in G
$$

The coboundary operator $\partial=\partial^{n}: X^{(n-1)} \longrightarrow X^{(n)}$ is defined for each $n \geq 1$ analogously to the case of left modules by

$$
\left(\partial^{n} x\right)\left(\sigma_{0}, \ldots, \sigma_{n}\right)=\prod_{i=0}^{n} x\left(\sigma_{0}, \ldots, \hat{\sigma}_{i}, \ldots, \sigma_{n}\right)^{(-1)^{i}},
$$

where the hat on $\sigma_{i}$ hints that $\sigma_{i}$ has to be omitted. We also set $\partial^{0}(a)$ as the constant function $G \longrightarrow A$ that maps each $\sigma \in G$ onto $a$. One proves that the sequence

$$
A \stackrel{\partial^{0}}{\longrightarrow} X^{(0)} \stackrel{\partial^{1}}{\longrightarrow} X^{(1)} \longrightarrow \cdots
$$

is exact ([NSW00], p. 10, Prop. 1.2.1). 
(B) Cochains ([NSW00], p. 11). We write $C^{n}=C^{n}(G, A)$ for the group of all $n$-dimensional homogeneous cochains, that is all $x \in X^{(n)}$ that are fixed by $G$. Thus, $x \in X^{(n)}$ is a homogeneous cochain if and only if it satisfies

$$
x\left(\sigma_{0}, \ldots, \sigma_{n}\right)^{\tau}=x\left(\sigma_{0} \tau, \ldots, \sigma_{n} \tau\right) \text { for all } \sigma_{0}, \ldots, \sigma_{n}, \tau \in G .
$$

Note that $\partial^{n}\left(C^{n-1}\right) \leq C^{n}$. Hence, the exact sequence 1.4.1) gives rise to a complex

$$
C^{0} \stackrel{\partial^{1}}{\longrightarrow} C^{1} \stackrel{\partial^{2}}{\longrightarrow} C^{2} \longrightarrow \cdots
$$

i.e. $\partial^{n+1} \circ \partial^{n}=1$. In particular, $\operatorname{Im}\left(\partial^{n}\right) \leq \operatorname{Ker}\left(\partial^{n+1}\right)$ for each $n \geq 0$. We write

$$
\begin{aligned}
& Z^{n}(G, A)=\operatorname{Ker}\left(\partial^{n+1}: C^{n}(G, A) \longrightarrow C^{n+1}(G, A)\right) \\
& B^{n}(G, A)=\operatorname{Im}\left(\partial^{n}: C^{n-1}(G, A) \longrightarrow C^{n}(G, A)\right)
\end{aligned}
$$

for the multiplicative groups of (homogeneous) cocycles and (homogeneous) coboundaries. By definition, $B^{0}(G, A)=1$ and we also have $B^{n}(G, A) \leq$ $Z^{n}(G, A)$, so we write $H^{n}(G, A)=Z^{n}(G, A) / B^{n}(G, A)$ for the $n$th cohomology group of the $G$-module $A$ with $H^{0}(G, A)=A^{G}=\left\{a \in A \mid a^{g}=\right.$ $a$ for each $g \in G\}$.

(C) Inhomogeneous cochains ([NSW00], p. 12). We write $\mathcal{C}^{0}=\mathcal{C}^{0}(G, A)=$ $A$ and $\mathcal{C}^{n}(G, A)=\operatorname{Map}_{\text {cont }}\left(G^{n}, A\right)$ for the group of all inhomogeneous $n$ cochains, $n=1,2, \ldots$. There is an isomorphism $C^{n} \longrightarrow \mathcal{C}^{n}$ that maps each $x \in C^{n}$ to the inhomogeneous cochains $y \in \mathcal{C}^{n}$ defined by

$y=x(1)$ for $n=0$ and $y\left(\sigma_{1}, \ldots, \sigma_{n}\right)=x\left(\sigma_{1} \sigma_{2}, \sigma_{1} \sigma_{2} \sigma_{3}, \ldots, \sigma_{1} \sigma_{2} \cdots \sigma_{n}, \sigma_{n}, 1\right)$ for $n \geq 1$.

The inverse map is defined by

$$
x\left(\sigma_{0}, \ldots, \sigma_{n}\right)=y\left(\sigma_{0} \sigma_{1}^{-1}, \sigma_{1} \sigma_{2}^{-1}, \ldots, \sigma_{n-1} \sigma_{n}^{-1}\right)^{\sigma_{n}} .
$$

The coboundary operation $\partial^{n}: C^{n-1} \longrightarrow C^{n}$ yields by this isomorphism a coboundary operation $\partial^{n}: \mathcal{C}^{n-1} \longrightarrow \mathcal{C}^{n}$ that have the following explicit 
(inhomogeneous) form:

$$
\begin{aligned}
\left(\partial^{n} y\right)\left(\sigma_{1}, \ldots, \sigma_{n}\right)= & y\left(\sigma_{1}, \ldots, \sigma_{n-1}\right)^{\sigma_{n}} \\
\cdot & y\left(\sigma_{2}, \ldots, \sigma_{n}\right)^{(-1)^{n}} \\
\cdot & \prod_{i=1}^{n-1} y\left(\sigma_{1}, \ldots, \sigma_{i-1}, \sigma_{i} \sigma_{i+1}, \sigma_{i+2}, \ldots, \sigma_{n}\right)^{(-1)^{i}} .
\end{aligned}
$$

In particular,

$$
\begin{aligned}
\left(\partial^{1} a\right)(\sigma) & =a^{\sigma} a^{-1} \text { for } a \in A=\mathcal{C}^{0} \\
\left(\partial^{2} y\right)\left(\sigma_{1}, \sigma_{2}\right) & =y\left(\sigma_{1}\right)^{\sigma_{2}} \cdot y\left(\sigma_{2}\right) \cdot y\left(\sigma_{1} \sigma_{2}\right)^{-1} \text { for } y \in \mathcal{C}^{1} .
\end{aligned}
$$

Setting

$$
\begin{aligned}
\mathcal{Z}^{n}(G, A) & =\operatorname{Ker}\left(\partial^{n+1}: \mathcal{C}^{n}(G, A) \longrightarrow \mathcal{C}^{n+1}(G, A)\right) \\
\mathcal{B}^{n}(G, A) & =\operatorname{Im}\left(\partial^{n}: \mathcal{C}^{n-1}(G, A) \longrightarrow \mathcal{C}^{n}(G, A)\right),
\end{aligned}
$$

the isomorphisms $C^{n}(G, A) \longrightarrow \mathcal{C}^{n}(G, A)$ yield isomorphisms

$$
H^{n}(G, A) \cong \mathcal{Z}^{n}(G, A) / \mathcal{B}^{n}(G, A)
$$

The groups of cochains $C^{n}(G, A)$ (resp. $\left.\mathcal{C}^{n}(G, A)\right)$ are functors in $A$. The boundary operators $\partial: C^{n-1}(G, A) \longrightarrow C^{n}(G, A)$ are morphisms between these functors ([NSW00], p. 34, §3). Hence, also the coboundary groups $B^{n}(G, A)$ and the cocycles groups $Z^{n}(G, A)$ are functorial in $A$.

As for left modules, each short exact sequence $0 \longrightarrow A \stackrel{\alpha}{\longrightarrow} B \stackrel{\beta}{\longrightarrow} C \longrightarrow 0$ of discrete $G$-module gives rise for each $n \geq 0$ to a long exact sequence of cohomology groups:

$$
\begin{aligned}
0 \rightarrow A^{G} & \rightarrow B^{G} \rightarrow C^{G} \stackrel{\delta}{\longrightarrow} H^{1}(G, A) \rightarrow \cdots \\
& \cdots \quad \rightarrow H^{n}(G, A) \rightarrow H^{n}(G, B) \rightarrow H^{n}(G, C) \stackrel{\delta}{\longrightarrow} H^{n+1}(G, A)
\end{aligned}
$$

where $\delta$ 's are the connecting homomorphisms ([NSW00], p. 26, Thm. 1.3.2).

In this work, particularly in chapter 4, we work with the inhomogeneous 
1-cocycles.

(D) The group $H^{1}(G, A)$ ([NSW00], p. 16). By definitions of $\mathcal{Z}^{1}(G, A)$ and $\mathcal{B}^{1}(G, A)$ and from 1.4.5), the inhomogeneous 1-cocycles (crossed homomorphisms) are functions $\chi: G \longrightarrow A$ such that

$$
\chi(\sigma \tau)=\chi(\sigma)^{\tau} \chi(\tau) \text { for all } \sigma, \tau \in G,
$$

and the inhomogeneous 1-coboundaries are the functions that satisfy

$$
\chi(\sigma)=a^{\sigma} a^{-1} \text { for all } \sigma \in G,
$$

for some $a \in A$.

Now we assume for the rest of this section that $A$ is a finite $G$-module. If $G$ acts trivially on $A$, then $H^{1}(G, A)=\operatorname{Hom}(G, A)$. Since $\operatorname{Aut}(A)$ is finite and the map $G \rightarrow \operatorname{Aut}(A)$ is continuous, $G$ has an open normal subgroup $N$ such that each $\nu \in N$ acts trivially on $A$. We claim that an abstract crossed homomorphism $\chi$ is continuous exactly when $\operatorname{Ker}(\chi)$ is an open subgroup of $G$. Indeed, in this case, $H=N \cap \operatorname{Ker}(\chi)$ is an open subgroup of $G$. Given $\eta \in H$ and $\sigma \in G$, we have $\chi(\sigma \eta)=\chi(\sigma)^{\eta} \chi(\eta)=\chi(\sigma)$. It follows that $\chi^{-1}(a)$ is an open subset of $G$ for each $a \in A$, as claimed.

(E) Special Mappings ([Rib70], p. 106-134). Let $g$ : $G \longrightarrow G^{\prime}$ be a continuous homomorphism of profinite groups, let $A$ (resp. $A^{\prime}$ ) be a $G$-module (resp. $G^{\prime}$-module), and let $f: A^{\prime} \longrightarrow A$ be a group homomorphism. We say that $g$ and $f$ are compatible maps if

$$
f\left(\left(a^{\prime}\right)^{g(\sigma)}\right)=f\left(a^{\prime}\right)^{\sigma} \text { for all } \sigma \in G \text { and } a^{\prime} \in A^{\prime} .
$$

(1.4.6) Each pair of compatible maps $g, f$ yields homomorphisms of the groups of cochains $(g, f): \mathcal{C}^{n}\left(G^{\prime}, A^{\prime}\right) \longrightarrow \mathcal{C}^{n}(G, A)$, for $n \geq 0$, defined by

$$
(g, f)\left(x^{\prime}\right)\left(\sigma_{1}, \ldots, \sigma_{n}\right)=f\left(x^{\prime}\left(g\left(\sigma_{1}\right), \ldots, g\left(\sigma_{n}\right)\right)\right) .
$$


(1.4.7) The homomorphism $(g, f)$ commutes with $\partial$ and therefore induces homomorphisms $H^{n}\left(G^{\prime}, A^{\prime}\right) \longrightarrow H^{n}(G, A)$ for $n \geq 0$.

(F) The restriction map. If $H$ is a closed subgroup of $G$ and $A$ a $G$ module, then $A$ is an $H$-module. Consider the compatible maps $H \hookrightarrow G$ and $A \stackrel{\text { id }}{\longrightarrow} A$. Then, (1.4.6) and (1.4.7) give rise to homomorphisms

$$
\text { res: } H^{n}(G, A) \longrightarrow H^{n}(H, A), n \geq 0
$$

called the restriction maps. The restriction maps are functorial in the $G$-modules, and commute with the connecting homomorphisms ([NSW00], p. 46, Prop. 1.5.2). That is, if $0 \rightarrow A \stackrel{\alpha}{\longrightarrow} B \stackrel{\beta}{\longrightarrow} C \rightarrow 0$ is a short exact sequence of $G$-modules, then the diagram

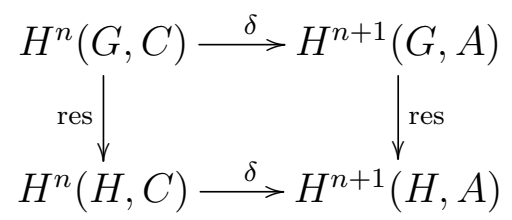

is commutative. For $n=1$, if $x \in H^{1}(G, A)$ is represented by $\chi: G \longrightarrow A$, then $\left.x\right|_{H}=\operatorname{res}(x) \in H^{1}(H, A)$ is represented by $\left.\chi\right|_{H}: H \longrightarrow A$, with $\left.\chi\right|_{H}(\sigma)=\chi(\sigma)$ for all $\sigma \in H$.

(G) The inflation map. Let $H$ be an open normal subgroup of $G$ and let $A$ be a $G$-module. Then $G / H$ acts continuously on $A^{H}$ by $a^{\sigma H}=a^{\sigma}$ for each $\sigma \in G$ and $a \in A^{H}$, so $A^{H}$ is a $G / H$-module. The projection $G \longrightarrow G / H$ and the inclusion $A^{H} \hookrightarrow A$ are compatible maps. Hence, again by (1.4.6) and (1.4.7), they induce homomorphisms

$$
\text { inf: } H^{n}\left(G / H, A^{H}\right) \longrightarrow H^{n}(G, A), n \geq 0
$$

called inflation maps.

For $n=1$, if $\bar{x} \in H^{1}\left(G / H, A^{H}\right)$ and $\bar{\chi}: G / H \longrightarrow A^{H}$ its representative, then $\chi: G \longrightarrow G / H \stackrel{\bar{\chi}}{\longrightarrow} A^{H} \hookrightarrow A$ is a representative of $\inf (x) \in H^{1}(G, A)$. Furthermore, we have the following exact sequence ([NSW00], p. 66, Prop. 1.6.6):

$$
1 \longrightarrow H^{1}\left(G / H, A^{H}\right) \stackrel{\inf }{\longrightarrow} H^{1}(G, A) \stackrel{\text { res }}{\longrightarrow} H^{1}(H, A) \text {. }
$$




\section{Chapter 2}

\section{Bound on the ramification of homomorphisms}

In this chapter, we construct a continuous homomorphism $h: \operatorname{Gal}(K) \longrightarrow A$, where $A=C_{l}^{r}$ for some prime number $l$ and a positive integer $r$, which coincides with some given local homomorphisms $h_{\mathfrak{P}}: \operatorname{Gal}\left(\hat{K}_{\mathfrak{P}}\right) \longrightarrow A$, where $\mathfrak{P}$ ranges over a finite set $S$ of primes of $K$. Moreover, we give a bound on the ramification of $h$ that depends on the finite set $S$ and on $r$.

The construction of $h$ as above is an essential ingredient in solving a finite embedding problem with solvable kernel and with a bound on the ramification. The first step for this type of embedding problem is to solve an embedding problem with an abelian kernel. Let $K / K_{0}$ be a Galois extension of number fields. Consider the embedding problem

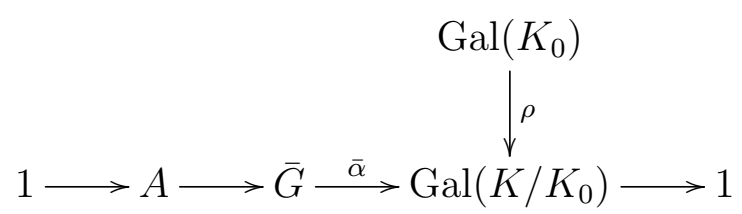

where $A$ is a simple $\operatorname{Gal}\left(K / K_{0}\right)$-module. One solves $(2.0 .1)$ with bounded ramification in two steps. The first step provides a solution $\psi_{0}: \operatorname{Gal}\left(K_{0}\right) \longrightarrow$ $\bar{G}$ without giving any bound on the ramification. In the second step we multiply $\psi_{0}$ by a crossed homomorphism $\chi: \operatorname{Gal}\left(K_{0}\right) \longrightarrow A$ and obtain the desired 
solution $\bar{\psi}=\psi_{0} \cdot \chi$ with an explicit bound on the ramification. For that, the ramification of $\chi$ itself has to be bounded. We construct $\chi$ in Chapter 3 as the image of $h$ under a certain corestriction map, multiplied by another suitable crossed homomorphism. Both $\chi$ and $h$ ramify at most at the same number of primes.

As always, all of our homomorphisms and crossed homomorphisms need to be continuous.

\subsection{Preliminary Result}

When solving an embedding problem with solvable kernel with bounded ramification, the solution $\bar{\psi}$ of the first step, i.e. an embedding problem with abelian kernel, must satisfy some conditions that ensure the solvability of the resulting embedding problem in the next step. First it has to be compatible with finitely many local solutions which are given in advance. Next, we have to ensure that each local embedding problem arising in the next step is solvable. We do this by using Lemma 1.2.6 and Lemma 1.2.7. This forces us to ensure that each ramified local embedding problem of the next step is cyclic. Finally, we have to bound the ramification of $\bar{\psi}$ (see Def. 1.2.4(b)). These three conditions are inherited by the solution $\bar{\psi}$ from the homomorphism $h$ that we construct in this chapter.

We say that a Galois extension $L / K$ is an $l$-extension if the order of the Galois group $\operatorname{Gal}(L / K)$ is a power of $l$.

We improve the following result in Lemma 2.3.5, in terms of the choice of the prime $\mathfrak{Q} \in \mathbb{P}(K)$, in order to construct our homomorphism $h$ that satisfies the three conditions described above.

Lemma 2.1.1. (GeJ98], Lem. 7.1) Let $S$ be a finite set of primes of $K$ which contains the basic set $S_{0}$ (Setup 1.3.1). Let $L$ be a finite $l$-extension 
of $K$ and let $n$ be a positive integer. For each $\mathfrak{P} \in S$ let $h_{\mathfrak{P}}: \hat{K}_{\mathfrak{P}} \longrightarrow C_{l}$ be a homomorphism. Suppose $\zeta_{l} \notin K$. Then there exists a prime $\mathfrak{Q} \in \mathbb{P}(K) \backslash S$ and there exists a continuous homomorphism $h: C_{K} \longrightarrow C_{l}$ such that

(a) $L\left(\zeta_{l^{n}}\right) \subseteq \hat{K}_{\mathfrak{Q}}$

(b) for each $\mathfrak{P} \in S,\left.h\right|_{\hat{K}_{\mathfrak{P}}}=h_{\mathfrak{P}}$,

(c) $h\left(U_{\mathfrak{Q}}\right)=C_{l}$,

(d) for each $\mathfrak{P} \in \mathbb{P}(K) \backslash(S \sqcup\{\mathfrak{Q}\}), h\left(U_{\mathfrak{P}}\right)=1$.

\subsection{Isomorphism of $\operatorname{Hom}(\operatorname{Gal}(K), A)$ and $\operatorname{Hom}\left(C_{K}, A\right)$}

The construction of the homomorphism $h: \operatorname{Gal}(K) \rightarrow A$ with $A \cong C_{l}^{n}$ starts with the construction of a homomorphism $\bar{h}: C_{K} \rightarrow A$, as in Lemma 2.1.1, where $C_{K}$ is the idele class group of $K$. An application of a natural isomorphism $\psi: \operatorname{Hom}(\operatorname{Gal}(K), A) \longrightarrow \operatorname{Hom}\left(C_{K}, A\right)$ then gives the desired $h$. In this section we define the isomorphism $\psi$ by using the reciprocity law (Section $1.3)$.

Every homomorphism from $C_{K}$ and $\hat{K}_{\mathfrak{P}}^{\times}$(as a subgroup of $C_{K}$ ) is tacitly assumed to be continuous. We identity the Galois group $\operatorname{Gal}\left(\hat{K}_{\mathfrak{P}}\right)$ with the Galois group $\operatorname{Gal}\left(K_{\mathfrak{P}}\right)$ as a subgroup of $\operatorname{Gal}(K)$. Let Hom be the functor of continuous homomorphisms. The local and global reciprocity law give rise to the following commutative diagram.

Lemma 2.2.1. Let $A$ be a finite abelian group and let $\mathfrak{P}$ be a prime of $K$. Then there is a commutative diagram

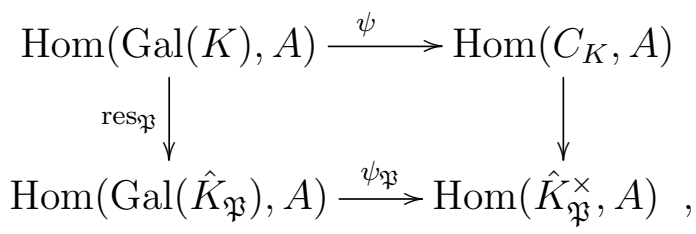


where the left vertical map is the restriction map defined by $\operatorname{res}_{\mathfrak{P}}(h)(\sigma)=$ $h\left(\sigma_{\mathfrak{P}}^{\lambda_{\mathfrak{P}}^{-1}}\right)$ for each $h \in \operatorname{Hom}(\operatorname{Gal}(K), A)$ and $\sigma \in \operatorname{Gal}\left(\hat{K}_{\mathfrak{P}}\right)$ (see the definition of $\lambda_{\mathfrak{P}}$ in Section 1.2), the right vertical map is the natural restriction map, and the horizontal maps are the isomorphisms induced by the global reciprocity map and the local reciprocity map. Furthermore, if $\mathfrak{P}$ is finite and if $f \in$ $\operatorname{Hom}\left(\operatorname{Gal}\left(\hat{K}_{\mathfrak{P}}\right), A\right)$ then $\psi_{\mathfrak{P}}(f)\left(U_{\mathfrak{p}}\right)=f\left(\hat{I}_{\mathfrak{P}}\right)$.

Proof. Part A: Definition of the $\psi$. Let $\psi: \operatorname{Hom}(\operatorname{Gal}(K), A) \longrightarrow \operatorname{Hom}\left(C_{K}, A\right)$ be the map defined by $\psi(f)=f_{1}$ for a homomorphism $f: \operatorname{Gal}(K) \longrightarrow A$, where $f_{1}: C_{K} \stackrel{\left(, L_{1} / K\right)}{\longrightarrow} \operatorname{Gal}\left(L_{1} / K\right) \stackrel{\bar{f}_{1}}{\longrightarrow} A$ such that $L_{1} / K$ is an arbitrary finite abelian extension with $f\left(\operatorname{Gal}\left(L_{1}\right)\right)=1$ and $\bar{f}_{1}: \operatorname{Gal}\left(L_{1} / K\right) \longrightarrow A$ is the unique homomorphism satisfying $\bar{f}_{1} \circ \operatorname{res}_{\tilde{K} / L_{1}}=f$ with $\operatorname{res}_{\tilde{K} / L_{1}}: \operatorname{Gal}(K) \longrightarrow$ $\operatorname{Gal}\left(L_{1} / K\right)$ the restriction map. The map $\psi$ is well defined. Indeed, let $L_{2} / K$ be another finite abelian extension with $f\left(\operatorname{Gal}\left(L_{2}\right)\right)=1$, and let $f_{2}: C_{K} \stackrel{\left(, L_{2} / K\right)}{\longrightarrow} \operatorname{Gal}\left(L_{2} / K\right) \stackrel{\bar{f}_{2}}{\longrightarrow} A$ be the corresponding image. The extension $L_{1} L_{2} / K$ is also finite abelian and $f\left(\operatorname{Gal}\left(L_{1} L_{2}\right)\right)=1$, so we can consider the corresponding image $f_{12}: C_{K} \stackrel{\left(, L_{1} L_{2} / K\right)}{\longrightarrow} \operatorname{Gal}\left(L_{1} L_{2} / K\right) \stackrel{\bar{f}_{12}}{\longrightarrow} A$. Then, by Proposition 1.3.7, the following diagram commutes:

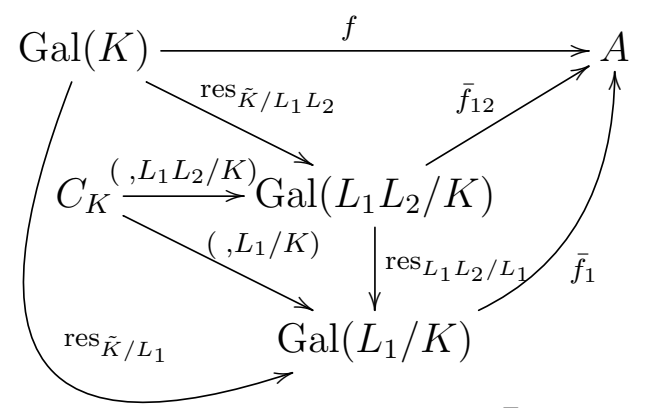

That is $f_{1}=\bar{f}_{1} \circ\left(, L_{1} / K\right)$ is equal to $f_{12}=\bar{f}_{12} \circ\left(, L_{1} L_{2} / K\right)$. Similarly $f_{2}=f_{12}$. Hence $f_{1}=f_{2}$ as desired.

Part B: The map $\psi$ is a homomorphism. Let $f, g \in \operatorname{Hom}(\operatorname{Gal}(K), A)$ and $L / K$ a finite abelian extension with $f(\operatorname{Gal}(L))=g(\operatorname{Gal}(L))=1$. Then $\psi(f): C_{K} \stackrel{(, L / K)}{\longrightarrow} \operatorname{Gal}(L / K) \stackrel{\bar{f}}{\longrightarrow} A$ and $\psi(g): C_{K} \stackrel{(, L / K)}{\longrightarrow} \operatorname{Gal}(L / K) \stackrel{\bar{g}}{\longrightarrow}$ A. Moreover $f g(\operatorname{Gal}(L))=1$, so $\psi(f g): C_{K} \stackrel{(, L / K)}{\longrightarrow} \operatorname{Gal}(L / K) \stackrel{\overline{f g}}{\longrightarrow} A$ with $\overline{f g}=\bar{f} \bar{g}$. It follows that for each $\alpha \in C_{K}, \psi(f g)(\alpha)=\bar{f} \bar{g}((\alpha, L / K))=$ 
$\bar{f}((\alpha, L / K)) \bar{g}((\alpha, L / K))=\psi(f)(\alpha) \psi(g)(\alpha)$. Therefore $\psi$ is a homomorphism.

Part C: Injectivity of $\psi$. Let $f \in \operatorname{Hom}(\operatorname{Gal}(K), A)$ and let $L$ be the fixed field of $\operatorname{Ker}(f)$, that is $\operatorname{Ker}(f)=\operatorname{Gal}(L)$. Then, $\operatorname{Gal}(L / K)$ is abelian. If $\psi(f)=1$, then for each $\alpha \in C_{K}, \bar{f}(\alpha, L / K)=1$. Since $(, L / K)$ is surjective (Theorem 1.3.4 , $\bar{f}=1$, so $f=1$. Therefore $\psi$ is injective.

Part D: Surjectivity of $\psi$. Let $f_{1}: C_{K} \longrightarrow A$ be a continuous homomorphism. In particular, $\operatorname{Ker}\left(f_{1}\right)$ is an open subgroup of $C_{K}$. By Theorem 1.3.5. $K$ has a finite abelian extension $L$ such that $N_{L / K} C_{L}=\operatorname{Ker}\left(f_{1}\right)$. Hence by Theorem 1.3.4. $(, L / K): C_{K} \longrightarrow \operatorname{Gal}(L / K)$ is an epimorphism whose kernel is $\operatorname{Ker}\left(f_{1}\right)$. Thus, there exists a homomorphism $\bar{f}: \operatorname{Gal}(L / K) \longrightarrow A$ such that $\bar{f} \circ(, L / K)=f_{1}$. Then by definition, $f=\bar{f} \circ$ res, with res: $\operatorname{Gal}(K) \longrightarrow$ $\operatorname{Gal}(L / K)$, satisfies $\psi(f)=f_{1}$. So $\psi$ is surjective.

Part E: Commutativity of the diagram. We define $\psi_{\mathfrak{P}}: \operatorname{Hom}\left(\operatorname{Gal}\left(\hat{K}_{\mathfrak{P}}\right), A\right) \longrightarrow$ $\operatorname{Hom}\left(\hat{K}_{\mathfrak{P}}, A\right)$ in a similar way to $\psi$ : for each continuous homomorphism $f \in \operatorname{Hom}\left(\operatorname{Gal}\left(\hat{K}_{\mathfrak{P}}\right), A\right)$ we choose a finite abelian extension $L$ of $\hat{K}_{\mathfrak{P}}$ such that $f(\operatorname{Gal}(L))=1$. Let $\bar{f}: \operatorname{Gal}\left(L / \hat{K}_{\mathfrak{P}}\right) \longrightarrow A$ be the unique homomorphism such that $\bar{f} \circ \operatorname{res}_{\widetilde{K}_{\mathfrak{P}} / L}=f$. Then, we set $\psi_{\mathfrak{P}}(f)=\bar{f} \circ\left(, L / \hat{K}_{\mathfrak{P}}\right)$. The proof that $\psi_{\mathfrak{P}}$ is a well defined isomorphism is done as for $\psi$, using the local class field theory (Theorem 1.3.6(a)). The commutativity of the diagram follows from Proposition 1.3.8.

Part F: We prove that $\psi(f)\left(U_{\mathfrak{P}}\right)=f\left(\hat{I}_{\mathfrak{P}}\right)$. For each $\alpha \in U_{\mathfrak{P}}$ we have by Theo$\operatorname{rem} 1.3 .6(\mathrm{~b})$, that $\left(\alpha, L / \hat{K}_{\mathfrak{P}}\right) \in I\left(L / \hat{K}_{\mathfrak{P}}\right)$. Let $\sigma \in \hat{I}_{\mathfrak{P}}$ with $\left.\sigma\right|_{L}=\left(\alpha, L / \hat{K}_{\mathfrak{P}}\right)$. Then

$$
\psi_{\mathfrak{P}}(f)(\alpha)=\bar{f}\left(\left(\alpha, L / \hat{K}_{\mathfrak{P}}\right)\right)=\bar{f}\left(\left.\sigma\right|_{L}\right)=f(\sigma) .
$$

Thus, $\psi_{\mathfrak{P}}(f)\left(U_{\mathfrak{P}}\right) \subseteq f\left(\hat{I}_{\mathfrak{P}}\right)$. 
Conversely, for each $\sigma \in \hat{I}_{\mathfrak{P}}$ there exists $\alpha \in U_{\mathfrak{P}}$ with $\left(\alpha, L / \hat{K}_{\mathfrak{P}}\right)=\left.\sigma\right|_{L}$. By 2.2.1, $f(\sigma)=\psi_{\mathfrak{P}}(f)(\alpha)$. Hence, $f\left(\hat{I}_{\mathfrak{P}}\right) \subseteq \psi_{\mathfrak{P}}(f)\left(U_{\mathfrak{P}}\right)$, as claimed.

\subsection{Construction of $h \in \operatorname{Hom}\left(\operatorname{Gal}(K), C_{l}\right)$}

Let $K / K_{0}$ be a finite Galois extension of number fields. For every finite set $S$ of primes of $K$ with $S_{0} \subseteq S$ and homomorphisms $h_{\mathfrak{P}}: \hat{K}_{\mathfrak{P}}^{\times} \longrightarrow C_{l}$ for each $\mathfrak{P} \in S$, we construct a continuous homomorphism $h: C_{K} \longrightarrow C_{l}$ such that $\left.h\right|_{\hat{K}_{\mathfrak{P}}^{\times}}=h_{\mathfrak{P}}$ for each $\mathfrak{P} \in S$ and $h\left(U_{\mathfrak{P}}\right)=1$ for each $\mathfrak{P} \in \mathbb{P}_{K} \backslash(S \sqcup\{\mathfrak{Q}\})$ for some $\mathfrak{Q} \in \mathbb{P}_{K} \backslash S$. The construction is carried out as in Lemma 2.1.1. However, we first choose a prime $\mathfrak{q} \in \mathbb{P}\left(K_{0}\right)$ and a prime $\mathfrak{Q} \in \mathbb{P}(K)$ that lies over $\mathfrak{q}$ and satisfies the conditions of the Lemma. This choice gives us the freedom we need in the solution of an embedding problem with kernel $C_{l}^{r}$ in Chapter 4. In the construction, we use the equality $I_{K}=I_{K, S} K^{\times}$, so we assume throughout this section that $S_{0} \subseteq S$.

Let $S$ be a finite set of primes of $K$. We say that $a_{1}, \ldots, a_{s} \in K_{S}$ are multiplicatively independent modulo $K_{S}^{l}$ if for all $l_{1}, \ldots, l_{s} \in \mathbb{Z}$ and $b \in K_{S}$, the equality $a_{1}^{l_{1}} \cdots a_{s}^{l_{s}}=b^{l}$ implies that $l \mid l_{i}$ for $i=1, \ldots, s$.

We start with some elementary results.

Lemma 2.3.1. (GeJ98], Lem. 5.2) Let $S$ be a finite set of primes of $K$ and $l$ a prime number. If $a_{1}, \ldots, a_{s} \in K_{S}$ are multiplicatively independent modulo $K_{S}^{l}$, then the fields $K\left(\zeta_{l}, \sqrt[l]{a_{1}}\right), \ldots, K\left(\zeta_{l}, \sqrt[l]{a_{s}}\right)$ are linearly disjoint and of degree $l$ over $K\left(\zeta_{l}\right)$. Let $n$ be a positive integer. If $L / K$ is an $l$ extension and $\zeta_{l} \notin K$, then the fields $L\left(\zeta_{l^{n}}, \sqrt[l]{a_{1}}\right), \ldots, L\left(\zeta_{l^{n}}, \sqrt[l]{a_{s}}\right)$ are linearly disjoint and of degree $l$ over $L\left(\zeta_{l^{n}}\right)$.

Lemma 2.3.2. Let $S$ be a finite set of primes of $K, l$ a prime number and assume that $\zeta_{l} \notin K$. Let $a_{1}, \ldots, a_{s}$ be multiplicatively independent elements of $K_{S}$ modulo $K_{S}^{l}$. Let $L$ be a an l-extension of $K$ and let $m$ be a 
positive integer. Let $M$ be a finite abelian extension of $K$. Then, the fields $L M\left(\zeta_{l^{m}}, \sqrt[l]{a_{1}}\right), \ldots, L M\left(\zeta_{l^{m}}, \sqrt[l]{a_{s}}\right)$ are linearly disjoint extensions of $L M\left(\zeta_{l^{m}}\right)$ of degree $l$.

Proof. We may write $M=M^{\prime} M^{\prime \prime}$, where $M^{\prime}$ is an abelian l-extension of $K$, and $M^{\prime \prime}$ is an abelian extension of $K$ whose degree is not divisible by $l$. Then, $L^{\prime}=L M^{\prime}$ is a finite $l$-extension of $K$. Applying Lemma 2.3.1 to $L^{\prime}$, we find that $L^{\prime}\left(\zeta_{l^{m}}, \sqrt[l]{a_{1}}\right), \ldots, L^{\prime}\left(\zeta_{l^{m}}, \sqrt[l]{a_{s}}\right)$ are linearly disjoint extensions of $L^{\prime}\left(\zeta_{l^{m}}\right)$ of degree $l$. In particular, $N=L^{\prime}\left(\zeta_{l^{m}}, \sqrt[l]{a_{1}}, \ldots, \sqrt[l]{a_{s}}\right)$ is an $l$-extension of $K$. Since $l \nmid\left[M^{\prime \prime}: K\right]$, the field $M^{\prime \prime}$ is linearly disjoint from $N$ over $K$. Hence $L M\left(\zeta_{l^{m}}, \sqrt[l]{a_{1}}\right), \ldots, L M\left(\zeta_{l^{m}}, \sqrt[l]{a_{s}}\right)$ are linearly disjoint extensions of $L M\left(\zeta_{l^{m}}\right)$ of degree $l$.

Remark 2.3.3. Let $n$ be a multiple of $l^{m}$. Using the notation of Lemma 2.3.2, suppose $L / K$ is a finite abelian $l$-extension. Then $M=L\left(\zeta_{n}\right)$ is a finite abelian extension of $K$. It follows that the fields

$$
L M\left(\zeta_{l^{m}}, \sqrt[l]{a_{1}}\right)=M\left(\sqrt[l]{a_{1}}\right), \ldots, M\left(\sqrt[l]{a_{s}}\right)=L\left(\zeta_{n}, \sqrt[l]{a_{s}}\right)
$$

are linearly disjoint extensions of $M\left(\zeta_{l^{m}}\right)=L\left(\zeta_{n}\right)$ of degree $l$.

Lemma 2.3.4. ([GeJ98], Lem. 4.1) Let $l$ be a prime number and $\mathfrak{Q} \in \mathbb{P}(K)$ with $\mathfrak{Q} \nmid l, \infty$. Suppose that $\zeta_{l} \in \hat{K}_{\mathfrak{Q}}$. Then $U_{\mathfrak{Q}} / U_{\mathfrak{Q}}^{l} \cong C_{l}$.

The following Lemma strengthens Lemma 2.1.1 in terms of the choice of the prime $\mathfrak{q}$. Specifically, GeJ98, chooses $\mathfrak{Q} \in \mathbb{P}(K)$ which totally splits in $L\left(\zeta_{l m}\right)$, with $L / K_{0}$ is a finite Galois extension such that $L / K$ is abelian $l$-extension. Instead, we choose a prime $\mathfrak{q} \in \mathbb{P}\left(K_{0}\right)$ that totally splits in $L\left(\zeta_{n}\right)$ for a multiple $n$ of $l^{m}$.

Lemma 2.3.5. Let $K_{0} \subseteq K \subseteq L$ be a tower of finite Galois extensions of number fields such that $L / K$ is abelian l-extension and $L / K_{0}$ is Galois. Suppose $\zeta_{l} \notin K$. Let $S$ be a finite set of primes of $K$ which contains $S_{0}$. Let $n=q l^{m}$ be a positive integer multiple of $l^{m}$ for some positive integers 
$q$ and $m$. For each $\mathfrak{P} \in S$ let $h_{\mathfrak{P}}: \hat{K}_{\mathfrak{P}}^{\times} \longrightarrow C_{l}$ be a homomorphism. Then there exists a prime $\left.\mathfrak{q} \in \mathbb{P}\left(K_{0}\right) \backslash S\right|_{K_{0}}$ and there exists a homomorphism $h: C_{K} \longrightarrow C_{l}$ such that:

(a) $\mathfrak{q}$ totally splits in $L\left(\zeta_{n}\right)$,

(b) $\left.h\right|_{\hat{K}_{\mathfrak{P}}^{\times}}=h_{\mathfrak{P}}$ for each $\mathfrak{P} \in S$,

(c) there exists $\mathfrak{Q} \in \mathbb{P}(K)$ such that $\left.\mathfrak{Q}\right|_{K_{0}}=\mathfrak{q}$ and $h\left(U_{\mathfrak{Q}}\right)=C_{l}$, and

(d) $h\left(U_{\mathfrak{P}}\right)=1$ for each $\mathfrak{P} \in \mathbb{P}(K) \backslash(S \sqcup\{\mathfrak{Q}\})$.

Proof. We break the proof into several parts.

PART A: Continuity. Each abstract homomorphism $h: C_{K} \rightarrow C_{l}$ that satisfies (a), (b), (c), and (d) is continuous, hence a homomorphism in the sense of Section 1.1. Indeed, by $(\mathrm{d}), h\left(U_{\mathfrak{P}}\right)=1$ for each $\mathfrak{P} \in \mathbb{P}(K) \backslash(S \bullet\{\mathfrak{Q}\})$. By (b), $\left.h\right|_{\hat{K}_{\mathfrak{P}}^{\times}}=h_{\mathfrak{P}}$ is continuous for each $\mathfrak{P} \in S$. Hence, by the statement $(1.3 .1)$ it suffices to prove that $\left.h\right|_{\hat{K}_{\mathfrak{Q}}^{\times}}$is continuous.

Since $U_{\mathfrak{Q}}$ is a profinite group (Section 1.1) and raising elements to the $l$ th power is continuous, $U_{\mathfrak{Q}}^{l}$ is closed in $U_{\mathfrak{Q}}$. Since each prime of $K$ dividing $l$ is in $S_{0} \subseteq S\left(\operatorname{Setup} 1.3 .1\right.$ ), $\mathfrak{Q} \nmid l, \infty$. By (a), $\zeta_{l} \in \hat{K}_{\mathfrak{Q}}^{\times}$. Hence, by Lemma 2.3.4. $U_{\mathfrak{Q}} / U_{\mathfrak{Q}}^{l} \cong C_{l}$. Therefore, $U_{\mathfrak{Q}}^{l}$ is an open subgroup of $U_{\mathfrak{Q}}$. Let $\pi_{\mathfrak{Q}}$ be a prime element of the valuation ring of $\hat{K}_{\mathfrak{Q}}$. Then, $\hat{K}_{\mathfrak{Q}}^{\times} \cong\left\langle\pi_{\mathfrak{Q}}\right\rangle \times U_{\mathfrak{Q}}$. Hence, by Section $1.1,\left(\hat{K}_{\mathfrak{Q}}^{\times}\right)^{l} \cong\left\langle\pi_{\mathfrak{Q}}^{l}\right\rangle \times U_{\mathfrak{Q}}^{l}$ is an open subgroup of $\hat{K}_{\mathfrak{Q}}^{\times}$. Finally, there is a commutative diagram

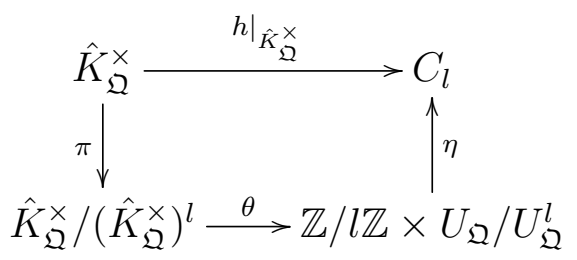

where $\pi$ is the quotient map, $\theta$ is an isomorphism, and $\eta$ is a homomorphism. Since $\left(\hat{K}_{\mathfrak{Q}}^{\times}\right)^{l}$ is an open subgroup of $\hat{K}_{\mathfrak{Q}}^{\times}, \pi$ is continuous. Since all groups 
appearing in the diagram but $\hat{K}_{\mathfrak{Q}}^{\times}$are finite, $\theta$ and $\eta$ are continuous. It follows that $\left.h\right|_{\hat{K}_{\mathfrak{Q}}^{\times}}$is continuous, as claimed.

PART B: Reduction of the lemma to constructing a continuous homomorphism $g: \overline{I_{K, S}} / \overline{K_{S}} \longrightarrow C_{l}$. By the unit theorem, $K_{S}$ is finitely generated ([CaF67], p. 72), hence $\left(K_{S}: K_{S}^{l}\right)=l^{s}$ for some positive integer $s$. Choose multiplicatively independent generators $a_{1}, \ldots, a_{s}$ of $K_{S}$ modulo $K_{S}^{l}$. For each $\mathfrak{Q} \in \mathbb{P}(K) \backslash S$ we can decompose $I_{K, S}$ as

$$
I_{K, S}=\prod_{\mathfrak{P} \in S} \hat{K}_{\mathfrak{P}}^{\times} \times U_{\mathfrak{Q}} \times \prod_{\mathfrak{P} \notin S \cup\{\mathfrak{Q}\}} U_{\mathfrak{P}}
$$

Use a bar to denote the reduction of elements and subgroups of $I_{K, S}$ modulo $I_{K, S}^{l}$. In particular

$$
\overline{I_{K, S}}=\prod_{\mathfrak{P} \in S} \overline{\hat{K}_{\mathfrak{P}}^{\times}} \times \overline{U_{\mathfrak{Q}}} \times \prod_{\mathfrak{P} \notin S \cup\{\mathfrak{Q}\}} \overline{U_{\mathfrak{P}}}
$$

and

$$
\overline{K_{S}}=\left\langle\bar{a}_{1}, \ldots, \bar{a}_{s}\right\rangle .
$$

Also, for each $\mathfrak{P} \in S$ the homomorphism $h_{\mathfrak{P}}: \hat{K}_{\mathfrak{P}}^{\times} \longrightarrow C_{l}$ induces a homomorphism $\bar{h}_{\mathfrak{P}}: \overline{\hat{K}_{\mathfrak{P}}^{\times}} \longrightarrow C_{l}$. Since $\overline{I_{K, S}} / \overline{K_{S}}$ is a quotient of $C_{K}=I_{K, S} / K_{S}$ (Remark 1.3.3), it suffices to find a prime $\left.\mathfrak{q} \in \mathbb{P}\left(K_{0}\right) \backslash S\right|_{K_{0}}$ which satisfies (a) and to construct a homomorphism $g: \overline{I_{K, S}} \longrightarrow C_{l}$ such that

(2.3.3) $\left.g\right|_{\bar{K}_{\mathfrak{P} \times}}=\bar{h}_{\mathfrak{P}}$ for each $\mathfrak{P} \in S$,

(2.3.4) $g\left(\overline{U_{\mathfrak{Q}}}\right)=C_{l}$ for some prime $\mathfrak{Q} \in \mathbb{P}(K)$ that lies over $\mathfrak{q}$,

(2.3.5) $g\left(\overline{U_{\mathfrak{P}}}\right)=1$ for each $\mathfrak{P} \in \mathbb{P}(K) \backslash(S \sqcup\{\mathfrak{Q}\})$,

(2.3.6) $g\left(\bar{a}_{i}\right)=1$ for $i=1, \ldots, s$

By 2.3.2 and (2.3.6), $g$ will induce a homomorphism $\bar{g}: \overline{I_{K, S}} / \overline{K_{S}} \longrightarrow C_{l}$ which will compose with the quotient map $C_{K} \longrightarrow \overline{I_{K, S}} / \overline{K_{S}}$ to the desired 
homomorphism $h$.

PART C: Presentation of $\bar{a}_{i}$ as an idele. For each $i$ between 1 and $s$ and each $\mathfrak{P} \in \mathbb{P}(K)$, let $a_{i \mathfrak{P}}$ be $a_{i}$ considered as an element of $\hat{K}_{\mathfrak{P}}^{\times}$and let

$$
\delta_{i}=\prod_{\mathfrak{P} \in S} \bar{h}_{\mathfrak{P}}\left(\bar{a}_{i \mathfrak{P}}\right)
$$

If $\mathfrak{q} \in \mathbb{P}\left(K_{0}\right)$ satisfies (a) and $\mathfrak{q} \nmid l, \infty$, then we choose $\mathfrak{Q} \in \mathbb{P}(K)$ over $\mathfrak{q}$. Then, $\mathfrak{Q}$ totally splits in $L\left(\zeta_{l}\right)$. It follows that $a_{1}, \ldots, a_{s}, \zeta_{l} \in U_{\mathfrak{Q}}$ and $\overline{U_{\mathfrak{Q}}} \cong C_{l}$ (Lemma 2.3.4). Choose a generator $\bar{u}_{\mathfrak{Q}}$ of $\overline{U_{\mathfrak{Q}}}$. For each $i$ there exists then $0 \leq \beta_{i}<l$ such that $\bar{a}_{i \mathfrak{Q}}=\bar{u}_{\mathfrak{Q}}^{\beta_{i}}$. The representation of $\bar{a}_{i}$ as an idele will therefore take the form:

$$
\bar{a}_{i}=\prod_{\mathfrak{P} \in S} \bar{a}_{i \mathfrak{P}} \cdot \bar{u}_{\mathfrak{Q}}^{\beta_{i}} \cdot \prod_{\mathfrak{P} \notin S \cup\{\mathfrak{Q}\}} \bar{a}_{i \mathfrak{P}} \cdot
$$

Conditions (2.3.3) and (2.3.5) force that

$$
\begin{aligned}
& g\left(\bar{a}_{i \mathfrak{P}}\right)=\bar{h}_{\mathfrak{P}}\left(\bar{a}_{i \mathfrak{P}}\right) \text { for } \mathfrak{P} \in S \text { and } \\
& g\left(\bar{a}_{i \mathfrak{P}}\right)=1 \text { for } \mathfrak{P} \in \mathbb{P}(K) \backslash(S \cup\{\mathfrak{Q}\}) .
\end{aligned}
$$

Condition $(2.3 .4)$ is equivalent to $g\left(\bar{u}_{\mathfrak{Q}}\right) \neq 1$. We have therefore to choose $\mathfrak{q}$ such that, in addition to 2.3 .9 , (a) will hold and to define $g\left(\bar{u}_{\mathfrak{Q}}\right)$ as a non-trivial element of $C_{l}$ such that (2.3.6) will be satisfied.

Let $N$ be the Galois closure of $L\left(\zeta_{n}, \sqrt[l]{a_{1}}, \ldots, \sqrt[l]{a_{s}}\right)$ over $K_{0}$. If $\delta_{i}=1$ for $i=1, \ldots, s$, we may use the Chebotarev density theorem to choose $\left.\mathfrak{q} \in \mathbb{P}\left(K_{0}\right) \backslash S\right|_{K_{0}}$ such that

$$
N \subseteq \hat{K}_{0, \mathfrak{q}}
$$

In particular (a) holds. We choose $\mathfrak{Q} \in \mathbb{P}(K)$ with $\left.\mathfrak{Q}\right|_{K_{0}}=\mathfrak{q}$. By its choice, $\mathfrak{q}$ totally splits in $K$, hence $\hat{K}_{\mathfrak{Q}}=\hat{K}_{0, \mathfrak{q}}$. It follows that $a_{i \mathfrak{Q}} \in U_{\mathfrak{Q}}^{l}$, so $\beta_{i}=0$ for $i=1, \ldots, s$. We therefore define $g\left(\bar{u}_{\mathfrak{Q}}\right)$ to be a generator of $C_{l}$ and derive from 2.3.8), 2.3.7) and 2.3.9 that $g\left(\bar{a}_{i}\right)=\delta_{i} \cdot g\left(\bar{u}_{\mathfrak{Q}}\right)^{\beta_{i}}=1$, so that $(2.3 .6)$ 
holds.

PART D: The main case. Having settled the case where $\delta_{i}=1$ for $i=$ $1, \ldots, s$, we may and we will from now on assume that

$$
\delta_{1} \neq 1
$$

Under this assumption there exists $0 \leq \varepsilon_{i}<l$ such that in $C_{l}$

$$
\delta_{1}^{\varepsilon_{i}}=\delta_{i}, \quad i=1, \ldots, s .
$$

In particular $\varepsilon_{1}=1$. Define

$$
b_{1}=a_{1} \text { and } b_{i}=a_{i} / a_{1}^{\varepsilon_{i}} \text {, for } i=2, \ldots, s .
$$

Since $a_{1}, \ldots, a_{s}$ are multiplicatively independent modulo $K_{S}^{l}$, so are $b_{1}, \ldots, b_{s}$. By Remark 2.3.3, $L\left(\zeta_{n}, \sqrt[l]{b_{1}}\right), \ldots, L\left(\zeta_{n}, \sqrt[l]{b_{s}}\right)$ are linearly disjoint fields of degree $l$ over $L\left(\zeta_{n}\right)$.

PART E: Choosing q. Part D allows us to choose $\sigma \in \operatorname{Gal}\left(N / L\left(\zeta_{n}\right)\right)$ with $\left(\sqrt[l]{a_{1}}\right)^{\sigma}=\zeta_{l} \sqrt[l]{a_{1}}$ and $\left(\sqrt[l]{b_{i}}\right)^{\sigma}=\sqrt[l]{b_{i}}, i=2, \ldots, s$. Chebotarev density theorem gives us a prime $\left.\mathfrak{q} \in \mathbb{P}\left(K_{0}\right) \backslash S\right|_{K_{0}}$ such that $\left(\frac{N / K_{0}}{\mathfrak{q}}\right)=\operatorname{Con}(\sigma)$. Thus, $L\left(\zeta_{n}\right) \subseteq \hat{K}_{0, \mathfrak{q}}$, so (a) holds .

We choose $\mathfrak{Q} \in \mathbb{P}(K)$ with $\left.\mathfrak{Q}\right|_{K_{0}}=\mathfrak{q}$. Since $\mathfrak{q}$ is unramified in $N$, so is $\mathfrak{Q}$. Therefore $\hat{K}_{\mathfrak{Q}}\left(\sqrt[l]{a_{1}}\right) / \hat{K}_{\mathfrak{Q}}$ is an unramified extension. It follows that the Frobenius element over $\hat{K}_{\mathfrak{Q}}$ acts on $\sqrt[l]{a_{1}}$ as $\sigma$, in particular the Frobenius element does not fix $\sqrt[l]{a_{1}}$. This implies that $\left[\hat{K}_{\mathfrak{Q}}\left(\sqrt[l]{a_{1}}\right): \hat{K}_{\mathfrak{Q}}\right]=l$, in particular:

$$
a_{1} \in U_{\mathfrak{Q}} \backslash U_{\mathfrak{Q}}^{l}
$$

On the other hand $b_{i} \in U_{\mathfrak{Q}}^{l}$ and therefore, by 2.3.13,

$$
\bar{a}_{i \mathfrak{Q}}=\bar{a}_{1 \mathfrak{Q}}^{\varepsilon_{i}}, \quad i=2, \ldots, s .
$$

PART F: Definition of $g$. By (2.3.14) and the choice of $\bar{u}_{\mathfrak{Q}}$ in Part B,

$$
\bar{a}_{1 \mathfrak{Q}}=\bar{u}_{\mathfrak{Q}}^{\beta} \text { with } 0<\beta<l .
$$


We may therefore define $g\left(\bar{u}_{\mathfrak{Q}}\right)$ as the element of $C_{l}$ that satisfies

$$
g\left(\bar{u}_{\mathfrak{Q}}\right)^{\beta}=\delta_{1}^{-1}
$$

In particular, by 2.3.11, $g\left(\bar{u}_{\mathfrak{Q}}\right) \neq 1$. By 2.3.15 and 2.3.16), $\bar{a}_{i \mathfrak{Q}}=\bar{u}_{\mathfrak{Q}}^{\beta \varepsilon_{i}}$, $i=2, \ldots, s$. Since $\varepsilon_{1}=1$, the latter equality also holds for $i=1$. This gives 2.3.8 the following form:

$$
\bar{a}_{i}=\prod_{\mathfrak{P} \in S} \bar{a}_{i \mathfrak{P}} \cdot \bar{u}_{\mathfrak{Q}}^{\beta \varepsilon_{i}} \prod_{\mathfrak{P} \notin S \cup\{\mathfrak{Q}\}} \bar{a}_{i \mathfrak{P}} \cdot
$$

By 2.3.7) and (2.3.9),

$$
\prod_{\mathfrak{P} \in S} g\left(\bar{a}_{i \mathfrak{P}}\right)=\delta_{i} \text { and } \prod_{\mathfrak{P} \notin S \cup\{\mathfrak{Q}\}} g\left(\bar{a}_{i \mathfrak{P}}\right)=1 .
$$

Apply $g$ on (2.3.18) and use (2.3.19), 2.3.17) and (2.3.12) to get that

$$
g\left(\bar{a}_{i}\right)=\left(\prod_{\mathfrak{P} \in S} g\left(\bar{a}_{i \mathfrak{P}}\right)\right) \cdot g\left(\bar{u}_{\mathfrak{Q}}\right)^{\beta \varepsilon_{i}}=\delta_{i} \delta_{1}^{-\varepsilon_{i}}=1 .
$$

So (2.3.6) holds and the proof is complete.

Corollary 2.3.6. Let $K_{0} \subseteq K \subseteq L$ be a tower of Galois extension of number fields such that $L / K_{0}$ is Galois, $L / K$ is a finite abelian $l$-extension and $\zeta_{l} \notin K$. Let $S$ be a finite set of primes of $K$ which contains $S_{0}$. Let $n=q l^{m}$ be a multiple of $l^{m}$ for some positive integers $q$ and $m$. For homomorphisms $h_{\mathfrak{P}}: \operatorname{Gal}\left(\hat{K}_{\mathfrak{P}}\right) \longrightarrow C_{l}, \mathfrak{P} \in S$, there exists a prime $\left.\mathfrak{q} \in \mathbb{P}\left(K_{0}\right) \backslash S\right|_{K_{0}}$ and a homomorphism $h: \operatorname{Gal}(K) \longrightarrow C_{l}$ such that

(a) $\mathfrak{q}$ totally splits in $L\left(\zeta_{n}\right)$,

(b) $\operatorname{res}_{\mathfrak{P}}(h)=h_{\mathfrak{P}}$ for each $\mathfrak{P} \in S$, where $\operatorname{res}_{\mathfrak{P}}$ is defined in Lemma 2.2.1.

(c) there exists $\mathfrak{Q} \in \mathbb{P}(K)$ such that $\left.\mathfrak{Q}\right|_{K_{0}}=\mathfrak{q}$ and $\operatorname{res}_{\mathfrak{P}}(h)\left(\hat{I}_{\mathfrak{Q}}\right)=C_{l}$,

(d) $\operatorname{res}_{\mathfrak{P}}(h)\left(\hat{I}_{\mathfrak{P}}\right)=1$ for each $\mathfrak{P} \in \mathbb{P}(K) \backslash(S \cup\{\mathfrak{Q}\})$.

Proof. For each $\mathfrak{P} \in S$, let $h_{\mathfrak{P}}^{\prime}: \hat{K}_{\mathfrak{P}}^{\times} \longrightarrow C_{l}$ be the homomorphism that Lemma 2.2.1 attaches to $h_{\mathfrak{P}}$. An application of Lemma 2.3.5 to the system $\left(h_{\mathfrak{P}}^{\prime}\right)_{\mathfrak{P}}$, and again a use of Lemma 2.2.1 give us the desired prime $\mathfrak{q} \in \mathbb{P}\left(K_{0}\right) \backslash$ $\left.S\right|_{K_{0}}$ and the $h: \operatorname{Gal}(K) \longrightarrow C_{l}$. 


\subsection{Bound on the Ramification}

$$
\text { of } h \in \operatorname{Hom}(\operatorname{Gal}(K), A)
$$

This section contains the main result of this chapter: the construction of $h: \operatorname{Gal}(K) \longrightarrow C_{l}^{r}$ with a bound on its ramification. We repeat the construction in Corollary 2.3.6 $r$ times to obtain the continuous homomorphism $h: \operatorname{Gal}(K) \longrightarrow A$ with $A=C_{l}^{r}$, which is unramified at each $\mathfrak{P} \in$ $\mathbb{P}(K) \backslash\left(S \sqcup\left\{\mathfrak{Q}_{1}, \ldots, \mathfrak{Q}_{r}\right\}\right)$ for some $\mathfrak{Q}_{1}, \ldots, \mathfrak{Q}_{r} \in \mathbb{P}(K) \backslash S$.

Given a homomorphism $h_{\mathfrak{P}}: \operatorname{Gal}\left(\hat{K}_{\mathfrak{P}}\right) \rightarrow A$ for each $\mathfrak{P} \in S$, considering the system of homomorphisms $\left(h_{\mathfrak{P}, i}=\pi_{i} \circ h_{\mathfrak{P}}\right)_{\mathfrak{P} \in S}$ where $\pi_{i}: A \rightarrow C_{l, i}$ is the projection to the $i$ th factor, by using Corollary 2.3.6 we may choose a prime $\left.\mathfrak{q}_{i} \in \mathbb{P}\left(K_{0}\right) \backslash S\right|_{K_{0}}$ and construct a homomorphism $h_{i}: \operatorname{Gal}(K) \rightarrow C_{l, i}$ that satisfy conditions (a)-(d) of Corollary 2.3.6 for each $i=1, \ldots, r$. Then the homomorphism $h=\left(h_{1}, \ldots, h_{r}\right)$ will satisfy the conclusions of our main proposition (Proposition 2.4.1). However, for the next use (as in the proof of Proposition 3.3.5, we need to know information about $\operatorname{res}_{\mathfrak{Q}_{i, j}}(h)\left(\operatorname{Gal}\left(\hat{K}_{\mathfrak{Q}_{i, j}}\right)\right)$ for each prime $\mathfrak{Q}_{i, j}$ that lies over $\mathfrak{q}_{i}$. For that, in addition to the use of Corollary 2.3.6, we enlarge $S$ to $S \cup \bigcup_{t=1}^{i-1}\left\{\mathfrak{Q} \in \mathbb{P}(K)|\mathfrak{Q}|_{K_{0}}=\mathfrak{q}_{t}\right\}$ for each $i=1, \ldots, r$ and add in the system of given homomorphisms the trivial homomorphism $h_{\mathfrak{Q}}: \operatorname{Gal}\left(\hat{K}_{\mathfrak{Q}}\right) \rightarrow C_{l, i}$ for each $\mathfrak{Q} \in \bigcup_{t=1}^{i-1}\left\{\mathfrak{Q} \in \mathbb{P}(K)|\mathfrak{Q}|_{K_{0}}=\mathfrak{q}_{t}\right\}$.

Proposition 2.4.1. Let $K_{0} \subseteq K \subseteq L$ be a tower of finite Galois extensions of number fields such that $L / K_{0}$ is a Galois extension, $L / K$ is an abelian $l$-extension and $\zeta_{l} \notin K$. Let $n=q l^{m}$ for some positive integers $q$ and $m$, and let $A=C_{l, 1} \times \cdots \times C_{l, r}$ where each $C_{l, i}, i=1, \ldots, r$, is an isomorphic copy of $C_{l}$. Let $S$ be a finite set of primes of $K$ which contains $S_{0}$. For each $\mathfrak{P} \in S$ let $h_{\mathfrak{P}}: \operatorname{Gal}\left(\hat{K}_{\mathfrak{P}}\right) \longrightarrow A$ be a homomorphism. Then there exist distinct primes $\mathfrak{q}_{1}, \ldots,\left.\mathfrak{q}_{r} \in \mathbb{P}\left(K_{0}\right) \backslash S\right|_{K_{0}}$ and there exists a homomorphism $h: \operatorname{Gal}(K) \longrightarrow A$ such that

(a) $\mathfrak{q}_{i}$ totally splits in $L\left(\zeta_{n}\right)$ for $i=1, \ldots, r$, 
(b) $\operatorname{res}_{\mathfrak{P}}(h)=h_{\mathfrak{P}}$, for each $\mathfrak{P} \in S$,

(c) for $i=1, \ldots, r$ there exists $\mathfrak{Q}_{i} \in \mathbb{P}(K)$ with $\left.\mathfrak{Q}_{i}\right|_{K_{0}}=\mathfrak{q}_{i}$ such that $\operatorname{res}_{\mathfrak{Q}_{i}}(h)\left(\operatorname{Gal}\left(\hat{K}_{\mathfrak{Q}_{i}}\right)\right)=1 \times \cdots \times 1 \times C_{l, i} \times 1 \times \cdots \times 1$,

(d) For each $\mathfrak{Q} \neq \mathfrak{Q}_{i}$ that lies over $\mathfrak{q}_{i}, \operatorname{res}_{\mathfrak{Q}}(h)\left(\operatorname{Gal}\left(\hat{K}_{\mathfrak{Q}}\right)\right) \leq 1 \times \cdots \times 1 \times$ $C_{l, i} \times 1 \times \cdots \times 1$

(e) $\operatorname{res}_{\mathfrak{P}}(h)\left(\hat{I}_{\mathfrak{P}}\right)=1$ for each $\mathfrak{P} \in \mathbb{P}(K) \backslash\left(S \cup\left\{\mathfrak{Q}_{1}, \ldots, \mathfrak{Q}_{r}\right\}\right)$.

Proof. For each $i=1, \ldots, r$ and each $\mathfrak{P} \in S$ consider the compositum $h_{\mathfrak{P}, i}: \operatorname{Gal}\left(\hat{K}_{\mathfrak{P}}\right) \longrightarrow C_{l, i}$ of $h_{\mathfrak{P}}$ and the projection $A \longrightarrow C_{l, i}$. Then, for each $\mathfrak{P} \in S$

$$
h_{\mathfrak{P}}=\left(h_{\mathfrak{P}, 1}, \ldots, h_{\mathfrak{P}, r}\right)
$$

Part A: $i=1$. Consider the system of homomorphisms $\left(h_{\mathfrak{P}, 1}: \operatorname{Gal}\left(\hat{K}_{\mathfrak{P}}\right) \longrightarrow\right.$ $\left.C_{l, 1}\right)_{\mathfrak{P} \in S}$. By Corollary 2.3.6, there exists a prime $\left.\mathfrak{q}_{1} \in \mathbb{P}\left(K_{0}\right) \backslash S\right|_{K_{0}}$ and there exists a homomorphism $h_{1}: \operatorname{Gal}(K) \longrightarrow C_{l, 1}$ such that:

(2.4.2) $\mathfrak{q}_{1}$ totally splits in $L\left(\zeta_{n}\right)$,

(2.4.3) $\operatorname{res}_{\mathfrak{P}}\left(h_{1}\right)=h_{\mathfrak{P}, 1}$ for each $\mathfrak{P} \in S$,

(2.4.4) there exists $\mathfrak{Q}_{1} \in \mathbb{P}(K)$ with $\left.\mathfrak{Q}_{1}\right|_{K_{0}}=\mathfrak{q}_{1}$ such that $\operatorname{res}_{\mathfrak{Q}_{1}}\left(h_{1}\right)\left(\hat{I}_{\mathfrak{Q}_{1}}\right)=$ $C_{l, 1}$,

(2.4.5) $\operatorname{res}_{\mathfrak{P}}\left(h_{1}\right)\left(\hat{I}_{\mathfrak{P}}\right)=1$ for each $\mathfrak{P} \in \mathbb{P}(K) \backslash\left(S \cup\left\{\mathfrak{Q}_{1}\right\}\right)$.

Let $L_{1}$ be the fixed field of $\operatorname{Ker}\left(h_{1}\right)$ in $\tilde{K}$. Then, by (2.4.4), $L_{1} / K$ is a cyclic $l$-extension, so $L L_{1} / K$ is a finite abelian $l$-extension. It follows that the Galois closure $L_{1}^{\prime}$ of $L L_{1}$ over $K_{0}$ is also a finite abelian $l$-extension of $K$. By $(2.4 .2), \mathfrak{q}_{1}$ totally splits in $K$. Let $\mathfrak{Q}_{1}=\mathfrak{Q}_{1,1}, \mathfrak{Q}_{1,2}, \ldots, \mathfrak{Q}_{1, k} \in \mathbb{P}(K)$ be the primes lying over $\mathfrak{q}_{1}$ where $k=\left[K: K_{0}\right]$.

Part B: $i=2$. For each $j=1, \ldots, k$ let $h_{\mathfrak{Q}_{1, j}, 2}: \operatorname{Gal}\left(\hat{K}_{\mathfrak{Q}_{1, j}}\right) \longrightarrow C_{l, 2}$ be the trivial homomorphism. Consider the system of homomorphisms $\left(h_{\mathfrak{P}, 2}: \operatorname{Gal}\left(\hat{K}_{\mathfrak{P}}\right) \longrightarrow\right.$ 
$\left.C_{l, 2}\right)_{\mathfrak{P} \in S \cup\left\{\mathfrak{Q}_{1,1}, \ldots, \mathfrak{Q}_{1, k}\right\}}$, with $h_{\mathfrak{P}, 2}: \operatorname{Gal}\left(\hat{K}_{\mathfrak{P}}\right) \stackrel{h_{\mathfrak{P}}}{\longrightarrow} A \rightarrow C_{l, 2}$. Considering the tower of Galois extensions $K_{0} \subseteq K \subseteq L_{1}^{\prime}$, where $L_{1}^{\prime}$ is defined in Part A, again by Corollary 2.3.6, there exists $\mathfrak{q}_{2} \in \mathbb{P}\left(K_{0}\right) \backslash\left(\left.S\right|_{K_{0}} \cup\left\{\mathfrak{q}_{1}\right\}\right)$ and there exists a homomorphism $h_{2}: \operatorname{Gal}(K) \longrightarrow C_{l, 2}$ such that:

(2.4.6) $\mathfrak{q}_{2}$ totally splits in $L_{1}^{\prime}\left(\zeta_{n}\right)$,

(2.4.7) $\operatorname{res}_{\mathfrak{P}}\left(h_{2}\right)=h_{\mathfrak{P}, 2}$ for each $\mathfrak{P} \in S \cup\left\{\mathfrak{Q}_{1,1}, \ldots, \mathfrak{Q}_{1, k}\right\}$,

(2.4.8) there exists $\mathfrak{Q}_{2} \in \mathbb{P}(K)$ with $\left.\mathfrak{Q}_{2}\right|_{K_{0}}=\mathfrak{q}_{2}$ such that $\operatorname{res}_{\mathfrak{Q}_{2}}\left(h_{2}\right)\left(\hat{I}_{\mathfrak{Q}_{2}}\right)=$ $C_{l, 2}$,

(2.4.9) $\operatorname{res}_{\mathfrak{P}}\left(h_{2}\right)\left(\hat{I}_{\mathfrak{P}}\right)=1$ for each $\mathfrak{P} \in \mathbb{P}(K) \backslash\left(S \cup\left\{\mathfrak{Q}_{1,1}, \ldots, \mathfrak{Q}_{1, k}, \mathfrak{Q}_{2}\right\}\right)$.

The choice of the homomorphism $h_{\mathfrak{Q}_{1, j}, 2}$ to be trivial and (2.4.7) imply that

$$
\operatorname{res}_{\mathfrak{Q}_{1, j}}\left(h_{2}\right)\left(\operatorname{Gal}\left(\hat{K}_{\mathfrak{Q}_{1, j}}\right)\right)=1, \text { for each } j=1, \ldots, k
$$

In particular $\operatorname{res}_{\mathfrak{Q}_{1, j}}\left(h_{2}\right)\left(\hat{I}_{\mathfrak{Q}_{1, j}}\right)=1$. It follows from $(2.4 .9)$ that

(2.4.11) $\operatorname{res}_{\mathfrak{P}}\left(h_{2}\right)\left(\hat{I}_{\mathfrak{P}}\right)=1$ for each $\mathfrak{P} \in \mathbb{P}(K) \backslash\left(S \cup\left\{\mathfrak{Q}_{2}\right\}\right)$

Furthermore, by (2.4.6), $\mathfrak{q}_{2}$ totally splits in $L_{1}^{\prime}$ hence also in $L_{1}$. The later field is the fixed field of $\operatorname{Ker}\left(h_{1}\right)$ in $\tilde{K}$. Hence

(2.4.12) $\operatorname{res}_{\mathfrak{Q}}\left(h_{1}\right)\left(\operatorname{Gal}\left(\hat{K}_{\mathfrak{Q}}\right)\right)=1$ for each $\mathfrak{Q} \in \mathbb{P}(K)$ that lies over $\mathfrak{q}_{2}$.

Part C: Induction. Continuing this process inductively we find for each $i$ between 1 and $r$ a prime $\mathfrak{q}_{i} \in \mathbb{P}\left(K_{0}\right) \backslash\left(\left.S\right|_{K_{0}} \cup\left\{\mathfrak{q}_{1}, \ldots, \mathfrak{q}_{i-1}\right\}\right)$ and a homomorphism $h_{i}: \operatorname{Gal}(K) \longrightarrow C_{l, i}$ such that

(2.4.13) $\mathfrak{q}_{i}$ totally splits in $L_{i-1}^{\prime}\left(\zeta_{n}\right)$, where $L_{i-1}^{\prime}$ is the Galois closure of $L L_{1} \cdots L_{i-1}$ over $K_{0}$ with $L_{j}$ the fixed field of $\operatorname{Ker}\left(h_{j}\right)$ in $\tilde{K}$ for $j=1, \ldots, i-1$.

(2.4.14) $\operatorname{res}_{\mathfrak{P}}\left(h_{i}\right)=h_{\mathfrak{P}, i}$ for each $\mathfrak{P} \in S$, with $h_{\mathfrak{P}, i}: \operatorname{Gal}\left(\hat{K}_{\mathfrak{P}}\right) \stackrel{h_{\mathfrak{P}}}{\longrightarrow} A \rightarrow C_{l, i}$ for each $\mathfrak{P} \in S$, and $\operatorname{res}_{\mathfrak{P}}\left(h_{i}\right)=1$ for each $\mathfrak{P} \in \mathbb{P}(K)$ that lies over one of the primes $\mathfrak{q}_{1}, \ldots, \mathfrak{q}_{i-1}$, 
(2.4.15) there exists $\mathfrak{Q}_{i} \in \mathbb{P}(K)$ with $\left.\mathfrak{Q}_{i}\right|_{K_{0}}=\mathfrak{q}_{i}$ such that $\operatorname{res}_{\mathfrak{Q}_{i}}\left(h_{i}\right)\left(\hat{I}_{\mathfrak{Q}_{i}}\right)=$ $C_{l, i}$, and

(2.4.16) $\operatorname{ress}_{\mathfrak{P}}\left(h_{i}\right)\left(\hat{I}_{\mathfrak{P}}\right)=1$ for each $\mathfrak{P} \in \mathbb{P}(K) \backslash\left(S \cup\left\{\mathfrak{Q}_{i}\right\}\right)$

(2.4.17) As in (2.4.12), for each $t<i, \operatorname{res}_{\mathfrak{Q}}\left(h_{t}\right)\left(\operatorname{Gal}\left(\hat{K}_{\mathfrak{Q}}\right)\right)=1$ for each $\mathfrak{Q} \in$ $\mathbb{P}(K)$ that lies over $\mathfrak{q}_{i}$

Part D: Conclusion. We now prove that the homomorphism

$$
h:=\left(h_{1}, \ldots, h_{r}\right): \operatorname{Gal}(K) \longrightarrow C_{l, 1} \times \cdots \times C_{l, r}
$$

and the primes $\mathfrak{q}_{1}, \ldots, \mathfrak{q}_{r} \in \mathbb{P}\left(K_{0}\right)$ satisfy the conditions (a)-(e) of the Proposition.

We consider $i$ between 1 and $r$. By (2.4.13), $\mathfrak{q}_{i}$ totally splits in $L\left(\zeta_{n}\right)$, so (a) is satisfied. By (2.4.14), $\operatorname{res}_{\mathfrak{P}}\left(h_{i}\right)=h_{\mathfrak{P}, i}$ for each $\mathfrak{P} \in S$. Hence, by (2.4.1) $\operatorname{res}_{\mathfrak{P}}(h)=\left(h_{\mathfrak{P}, 1}, \ldots, h_{\mathfrak{P}, r}\right)=h_{\mathfrak{P}}$ for each $\mathfrak{P} \in S$, thus (b) is satisfied.

Now,

$$
\begin{aligned}
\operatorname{res}_{\mathfrak{Q}_{i}}(h)\left(\operatorname{Gal}\left(\hat{K}_{\mathfrak{Q}_{i}}\right)\right)= & \left(\operatorname{res}_{\mathfrak{Q}_{i}}\left(h_{1}\right)\left(\operatorname{Gal}\left(\hat{K}_{\mathfrak{Q}_{i}}\right)\right), \ldots, \operatorname{res}_{\mathfrak{Q}_{i}}\left(h_{i}\right)\left(\operatorname{Gal}\left(\hat{K}_{\mathfrak{Q}_{i}}\right)\right),\right. \\
& \left.\ldots, \operatorname{res}_{\mathfrak{Q}_{i}}\left(h_{r}\right)\left(\operatorname{Gal}\left(\hat{K}_{\mathfrak{Q}_{i}}\right)\right)\right)
\end{aligned}
$$

$\operatorname{By}(2.4 .15), \operatorname{res}_{\mathfrak{Q}_{i}}\left(h_{i}\right)\left(\hat{I}_{\mathfrak{Q}_{i}}\right)=C_{l, i}$ which implies

$$
\operatorname{res}_{\mathfrak{Q}_{i}}\left(h_{i}\right)\left(\operatorname{Gal}\left(\hat{K}_{\mathfrak{Q}_{i}}\right)\right)=C_{l, i}
$$

On the other hand, for $t<i$, by (2.4.17) we have

$$
\operatorname{res}_{\mathfrak{Q}_{i}}\left(h_{t}\right)\left(\operatorname{Gal}\left(\hat{K}_{\mathfrak{Q}_{i}}\right)\right)=1, \text { for } 1 \leq t \leq i-1
$$

Moreover, for $t>i,(2.4 .14)$ applied to $h_{t}$ implies that

$$
\operatorname{res}_{\mathfrak{Q}_{i}}\left(h_{t}\right)\left(\operatorname{Gal}\left(\hat{K}_{\mathfrak{Q}_{i}}\right)\right)=1, \quad t>i
$$

It follows from 2.4.18, 2.4.19, and 2.4.20 that

$$
\operatorname{res}_{\mathfrak{Q}_{i}}(h)\left(\operatorname{Gal}\left(\hat{K}_{\mathfrak{Q}_{i}}\right)\right)=1 \times \cdots \times C_{l, i} \times \cdots \times 1, \quad i=1, \ldots, r .
$$


Thus (c) is satisfied.

Let $\mathfrak{Q} \neq \mathfrak{Q}_{i}$ a prime that lies over $\mathfrak{q}_{i}$. We have

$$
\begin{aligned}
\operatorname{res}_{\mathfrak{Q}}(h)\left(\operatorname{Gal}\left(\hat{K}_{\mathfrak{Q}}\right)\right)= & \left(\operatorname{res}_{\mathfrak{Q}}\left(h_{1}\right)\left(\operatorname{Gal}\left(\hat{K}_{\mathfrak{Q}}\right)\right), \ldots, \operatorname{res}_{\mathfrak{Q}}\left(h_{i}\right)\left(\operatorname{Gal}\left(\hat{K}_{\mathfrak{Q}}\right)\right),\right. \\
& \left.\ldots, \operatorname{res}_{\mathfrak{Q}}\left(h_{r}\right)\left(\operatorname{Gal}\left(\hat{K}_{\mathfrak{Q}}\right)\right)\right) .
\end{aligned}
$$

If $t<i$ or $t>i$ by the same arguments as in in proof of (c)

$$
\operatorname{res}_{\mathfrak{Q}}\left(h_{t}\right)\left(\operatorname{Gal}\left(\hat{K}_{\mathfrak{Q}}\right)\right)=1
$$

Since the image of $h_{i}$ is a subgroup of $C_{l, i}$, we have

$$
\operatorname{res}_{\mathfrak{Q}}\left(h_{i}\right)\left(\operatorname{Gal}\left(\hat{K}_{\mathfrak{Q}}\right)\right) \leq C_{l, i}
$$

It follows from 2.4 .22 and 2.4 .23 , that $\operatorname{res}_{\mathfrak{Q}}(h)\left(\operatorname{Gal}\left(\hat{K}_{\mathfrak{Q}}\right)\right) \leq 1 \times \cdots \times 1 \times$ $C_{l, i} \times 1 \times \cdots \times 1$. So, $(\mathrm{d})$ is satisfies

Finally, for each $\mathfrak{P} \in \mathbb{P}(K) \backslash\left(S \cup\left\{\mathfrak{Q}_{1}, \ldots, \mathfrak{Q}_{r}\right\}\right)$, by $(2.4 .16)$, $\operatorname{ress}_{\mathfrak{P}}(h)\left(\hat{I}_{\mathfrak{P}}\right)=$ $\left(\operatorname{res}_{\mathfrak{P}}\left(h_{1}\right)\left(\hat{I}_{\mathfrak{P}}\right), \ldots, \operatorname{res}_{\mathfrak{P}}\left(h_{r}\right)\left(\hat{I}_{\mathfrak{P}}\right)\right)=1 \times \cdots \times 1$, so $(\mathrm{e})$ is satisfied, and this completes the proof of the proposition. 


\section{Chapter 3}

\section{Bound on the ramification of cohomology classes}

Let $K / K_{0}$ be a Galois extension of number fields. Given a simple $\operatorname{Gal}\left(K_{0}\right)$ module $A=C_{l}^{r}$ on which $\operatorname{Gal}(K)$ acts trivially, a finite set $T$ of primes of $K_{0}$, and an element $y_{\mathfrak{p}} \in H^{1}\left(\operatorname{Gal}\left(\hat{K}_{0, \mathfrak{p}}\right), A\right)$ for each $\mathfrak{p} \in T$, the aim of this chapter is to construct an element $x \in H^{1}\left(\mathrm{Gal}\left(K_{0}\right), A\right)$ which coincides with $y_{\mathfrak{p}}$ for each $\mathfrak{p} \in T$ and the number of the primes of $K_{0}$ where $x$ ramifies is bounded depending on $T$ and $r$. The construction is based on Theorem 1 of [Neu79, where no bound on the ramification is given.

If $\psi_{0}$ is a solution of the embedding problem

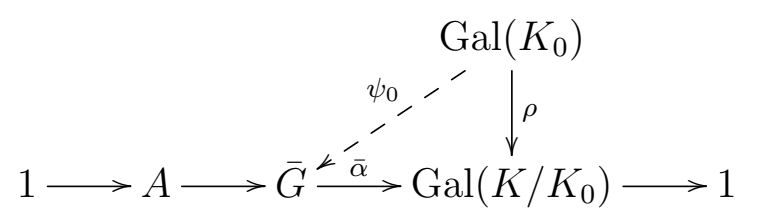

and $\chi: \operatorname{Gal}\left(K_{0}\right) \longrightarrow A$ is a crossed homomorphism, then $\bar{\psi}=\psi_{0} \cdot \chi$ is also a solution of (3.0.1) (Lemma 4.1.1). Our aim is to multiply $\psi_{0}$ by a suitable $\chi$ in order to give a bound on the ramification of $\bar{\psi}$ which will coincide with the bound on the ramification of $\chi$. 
The class $x \in H^{1}\left(\operatorname{Gal}\left(K_{0}\right), A\right)$ of the crossed homomorphism $\chi: \operatorname{Gal}\left(K_{0}\right) \rightarrow A$ is again constructed in a such a way that it coincides with a given local element of $H^{1}\left(\operatorname{Gal}\left(\hat{K}_{0, \mathfrak{p}}\right), A\right)$ for $\mathfrak{p} \in T$, it is cyclic at each prime where it ramifies, and we have a bound on its ramification. These conditions are similar to the required conditions in the construction of the homomorphism $h$ in Chapter 2. In fact, $x$ inherits these properties from $h$ by the corestriction map.

\subsection{Definitions and a Preliminary Result}

Let $K_{0}$ be a number field and let $A$ be a $\operatorname{Gal}\left(K_{0}\right)$-module. Let $\mathfrak{p} \in \mathbb{P}\left(K_{0}\right)$. We consider $A$ as a $\operatorname{Gal}\left(\hat{K}_{0, \mathfrak{p}}\right)$-module with $a^{\sigma}=a^{\left.\sigma\right|_{K_{0}}}$ for each $a \in A$ and $\sigma \in \operatorname{Gal}\left(\hat{K}_{0, \mathfrak{p}}\right)$ (by our construction of $K_{0, \mathfrak{p}}$ in Section 1.2 , if $\sigma \in \operatorname{Gal}\left(\hat{K}_{0, \mathfrak{p}}\right)$, then $\left.\operatorname{res}_{\tilde{K}_{0}}(\sigma) \in \operatorname{Gal}\left(K_{0, \mathfrak{p}}\right) \leq \operatorname{Gal}\left(K_{0}\right)\right)$.

Definition 3.1.1. For each $n \geq 0$ and $\mathfrak{p} \in \mathbb{P}\left(K_{0}\right)$, we define the restriction map res $\operatorname{res}_{\mathfrak{p}} H^{n}\left(\operatorname{Gal}\left(K_{0}\right), A\right) \longrightarrow H^{n}\left(\operatorname{Gal}\left(\hat{K}_{0, \mathfrak{p}}\right)\right)$ with $\operatorname{res}_{\mathfrak{p}}(u)\left(\sigma_{0}, \ldots, \sigma_{n}\right)=$ $u\left(\left.\sigma_{1}\right|_{\tilde{K}_{0}}, \ldots,\left.\sigma_{n}\right|_{\tilde{K}_{0}}\right)$ for each $u \in C^{n}\left(\operatorname{Gal}\left(K_{0}\right), A\right)$ and $\sigma_{0}, \ldots, \sigma_{n} \in \operatorname{Gal}\left(\hat{K}_{0, \mathfrak{p}}\right)^{n+1}$.

If $\mathfrak{p} \in \mathbb{P}_{\text {fin }}\left(K_{0}\right)$ we consider the inflation-restriction exact sequence given by 1.4.8:

$$
1 \longrightarrow H^{1}\left(\operatorname{Gal}\left(\hat{K}_{0, \mathfrak{p}}\right) / \hat{I}_{\mathfrak{p}}, A^{\hat{I}_{\mathfrak{p}}}\right) \stackrel{\inf }{\longrightarrow} H^{1}\left(\operatorname{Gal}\left(\hat{K}_{0, \mathfrak{p}}\right), A\right) \stackrel{\text { res }}{\longrightarrow} H^{1}\left(\hat{I}_{\mathfrak{p}}, A\right) .
$$

\section{Definition 3.1.2.}

(a) An element $x_{\mathfrak{p}} \in H^{1}\left(\operatorname{Gal}\left(\hat{K}_{0, \mathfrak{p}}\right), A\right)$ is unramified if $x_{\mathfrak{p}} \in \operatorname{Im}(\inf )$, alternatively, if $\operatorname{res}\left(x_{\mathfrak{p}}\right)=1$. That is, if $\chi_{\mathfrak{p}}: \operatorname{Gal}\left(\hat{K}_{0, \mathfrak{p}}\right) \longrightarrow A$ is a representative of $x_{\mathfrak{p}}$, then there exists $a \in A$ such that for all $\sigma \in$ $\hat{I}_{\mathfrak{p}}, \chi_{\mathfrak{p}}(\sigma)=a^{\sigma} a^{-1}$ (a coboundary representative in $\mathcal{Z}^{1}\left(\hat{I}_{\mathfrak{p}}, A\right)$ ). Note that if $\operatorname{Gal}\left(\hat{K}_{0, \mathfrak{p}}\right)$ acts trivially on $A$, then $x_{\mathfrak{p}}: \operatorname{Gal}\left(\hat{K}_{0, \mathfrak{p}}\right) \longrightarrow A$ is a homomorphism (Section $1.4(\mathrm{D})$ ). In this case, the definition of the unramification of $x_{\mathfrak{p}}$ at $\mathfrak{p}$ as an element of $H^{1}\left(\operatorname{Gal}\left(\hat{K}_{0, \mathfrak{p}}\right), A\right)$ coincides with the definition of $x_{\mathfrak{p}}$ being an unramified homomorphism, that is $x_{\mathfrak{p}}\left(\hat{I}_{\mathfrak{p}}\right)=1$ (Def. 1.2.4(b)). 
(b) An element $x_{\mathfrak{p}} \in H^{1}\left(\operatorname{Gal}\left(\hat{K}_{0, \mathfrak{p}}\right), A\right)$ is cyclic if there exists a cyclic extension $L_{\mathfrak{p}} / \hat{K}_{0, \mathfrak{p}}$ such that $x_{\mathfrak{p}}$ lies in the kernel of the restriction map res: $H^{1}\left(\operatorname{Gal}\left(\hat{K}_{0, \mathfrak{p}}\right), A\right) \longrightarrow H^{1}\left(\operatorname{Gal}\left(L_{\mathfrak{p}}\right), A\right)$.

If $\operatorname{Gal}\left(\hat{K}_{0, \mathfrak{p}}\right)$ acts trivially on $A$, then $x_{\mathfrak{p}}: \operatorname{Gal}\left(\hat{K}_{0, \mathfrak{p}}\right) \longrightarrow A$ is a homomorphism. In this case, $x_{\mathfrak{p}}$ is cyclic if it factors through a cyclic extension of $\hat{K}_{0, \mathfrak{p}}$ : there exits a cyclic extension $L_{\mathfrak{p}} / \hat{K}_{0, \mathfrak{p}}$ such that $\operatorname{Gal}\left(L_{\mathfrak{p}}\right) \leq \operatorname{Ker}\left(x_{\mathfrak{p}}\right)($ Def. $1.2 .4(\mathrm{c}))$.

The construction of $x \in H^{1}\left(\operatorname{Gal}\left(K_{0}\right), A\right)$ mentioned in the introduction to the present chapter is based on that of [Neu79] in the following local-global result for elements of $H^{1}\left(\mathrm{Gal}\left(K_{0}\right), A\right)$ :

Proposition 3.1.3 ([Neu79], Thm. 1). Let $A=C_{l}^{r}$ be a simple Gal $\left(K_{0}\right)$ module. Let $K / K_{0}$ be a finite Galois extension such that $\operatorname{Gal}(K)$ acts trivially on $A$, and $\zeta_{l} \notin K$. Let $\Omega / K$ be a finite abelian extension and consider a finite set $S$ of primes of $K_{0}$. For each $\mathfrak{p} \in S$, let $y_{\mathfrak{p}} \in H^{1}\left(\operatorname{Gal}\left(\hat{K}_{0, \mathfrak{p}}\right), A\right)$. Then, there exists an element $x \in H^{1}\left(\operatorname{Gal}\left(K_{0}\right), A\right)$ which satisfies the following conditions:

(a) $\operatorname{res}_{\mathfrak{p}}(x)=y_{\mathfrak{p}}$ for $\mathfrak{p} \in S$ (Def. 3.1.1).

(b) If $\mathfrak{p} \in \mathbb{P}\left(K_{0}\right) \backslash S$, then $\operatorname{res}_{\mathfrak{p}}(x)$ is cyclic. If in addition $\operatorname{res}_{\mathfrak{p}}(x)$ is ramified, then $\mathfrak{p}$ totally splits in $\Omega$.

We improve this result by giving a bound on the ramification of $x$ that depends on the set $S$ and on $r$. In addition, we take care of the cyclic property of $x_{\mathfrak{p}}$ at the primes where $x$ ramifies, but not for all $\mathfrak{p} \in \mathbb{P}\left(K_{0}\right) \backslash S$ as in the first part of condition (b) of the above proposition.

\subsection{Corestriction Map}

In general, if $G$ is a profinite group, $H$ an open subgroup of $G$, and $A$ a $G$-module, then the corestriction map lifts an element of $H^{1}(H, A)$ to an 
element of $H^{1}(G, A)$. We apply the corestriction map to the case where $G=\operatorname{Gal}\left(K_{0}\right), H=\operatorname{Gal}(K)$, and $A=C_{l}^{r}$ on which $\operatorname{Gal}(K)$ acts trivially (i.e. $\left.H^{1}(\operatorname{Gal}(K), A)=\operatorname{Hom}(\operatorname{Gal}(K), A)\right)$. Then, we can lift the homomorphism $h: \operatorname{Gal}(K) \rightarrow A$ constructed in Proposition 2.4.1 to an element of $H^{1}\left(\mathrm{Gal}\left(K_{0}\right), A\right)$ that plays a significant role in the construction of our desired element $x \in H^{1}\left(\operatorname{Gal}\left(K_{0}\right), A\right)$. The local cyclic property of $x$ at each prime where it ramifies, and the bound on its ramification are induced from that of $h$.

We start with the general definition. Let $G$ be a profinite group, $H$ an open subgroup of $G$, and $A$ a $G$-module. We choose a system $T$ of representatives for the left cosets of $G$ modulo $H$. Thus, $G=\bigcup_{\tau \in T} \tau H$. Then, for each $\sigma \in G$ there exists a unique element $\tilde{\sigma} \in T$ such that $\sigma H=\tilde{\sigma} H$. Hence, there exists a unique element $\eta_{\sigma} \in H$ such that $\sigma=\tilde{\sigma} \cdot \eta_{\sigma}$. In particular, for each $\eta \in H$ we have $\tilde{\sigma} H=\sigma H=\sigma \eta H=\widetilde{\sigma \eta} H$ so

$$
\tilde{\sigma}=\widetilde{\sigma \eta} \text {. }
$$

Having chosen $T$ we define for each $n \geq 0$ a homomorphism

$$
\text { cor }=\operatorname{cor}^{n}: C^{n}(H, A) \longrightarrow \operatorname{Map}_{\text {cont }}\left(G^{n+1}, A\right),
$$

called the corestriction map, by

$$
\begin{aligned}
\operatorname{cor}(x)\left(\sigma_{0}, \ldots, \sigma_{n}\right) & =\prod_{\tau \in T} x\left({\widetilde{\sigma_{0} \tau}}^{-1} \sigma_{0} \tau, \ldots,{\widetilde{\sigma_{n} \tau}}^{-1} \sigma_{n} \tau\right)^{\tau^{-1}} \\
& =\prod_{\tau \in T} x\left(\eta_{\sigma_{0} \tau}, \ldots, \eta_{\sigma_{n} \tau}\right)^{\tau^{-1}}
\end{aligned}
$$

Recall the $C^{n}(H, A)$ is the group of all $n$-dimensional homogeneous cochains (Section 1.4(B)). This homomorphism has the following properties:

(3.2.3) By the homogeneity 1.4.2 of the cochains and by 3.2.1), cor $(x)$ is independent of the choice of $T$.

(3.2.4) The image of each $x \in C^{n}(H, A)$ under cor is a homogeneous cochain (i.e. $\operatorname{cor}(x) \in C^{n}(G, A)$ ). Indeed, if $\sigma \in G$ and $T$ is a system of 
representatives of the left cosets of $G$ modulo $H$, then so is $\sigma T$. Hence by $(3.2 .2$ and by (3.2.3),

$$
\begin{aligned}
\operatorname{cor}(x)\left(\sigma_{0} \sigma, \ldots, \sigma_{n} \sigma\right)^{\sigma^{-1}} & =\prod_{\tau \in T}\left(x\left(\eta_{\sigma_{0} \sigma \tau}, \ldots, \eta_{\sigma_{n} \sigma \tau}\right)^{\tau^{-1}}\right)^{\sigma^{-1}} \\
& =\prod_{\tau \in T} x\left(\eta_{\sigma_{0} \sigma \tau}, \ldots, \eta_{\sigma_{n} \sigma \tau}\right)^{(\sigma \tau)^{-1}} \\
& =\operatorname{cor}(x)\left(\sigma_{0}, \ldots, \sigma_{n}\right)
\end{aligned}
$$

(3.2.5) By definition (3.2.2), the homomorphisms $\operatorname{cor}^{n}: C^{n}(H, A) \longrightarrow C^{n}(G, A)$ are functorial in $A$.

(3.2.6) The homogeneity condition 1.4.2 and the definition 3.2.2 imply that the corestriction maps are compatible with the coboundary operators (Section 1.4(A)), in other words cor $\circ \partial=\partial \circ$ cor. Therefore, the cochain homomorphisms cor $^{n}$ give rise to homomorphisms of cohomology groups cor $=\operatorname{cor}^{n}: H^{n}(H, A) \longrightarrow H^{n}(G, A)$.

(3.2.7) By 3.2 .2 , the homomorphism $\operatorname{cor}^{0}$ maps $A^{H}=H^{0}(H, A)$ into $A^{G}=$ $H^{0}(G, A)$. Indeed, for $a \in A^{H}$ we have $\operatorname{cor}^{0}(a)=\prod_{\tau \in T} a^{\tau^{-1}}=$ $\prod_{\tau^{\prime} \in T^{\prime}} a^{\tau^{\prime}}$, where $T^{\prime}=\left\{\tau^{-1} \mid \tau \in T\right\}$. Note that $G=\bigcup_{\tau^{\prime} \in T^{\prime}} H \tau^{\prime}$, so cor $^{0}$ coincides with the relative norm norm $_{G / H}: A^{H} \longrightarrow A^{G}$.

(3.2.8) The corestriction maps commute with the connecting homomorphisms $\delta$ of cohomology groups. In other words, let $0 \rightarrow A \stackrel{\alpha}{\longrightarrow} B \stackrel{\beta}{\longrightarrow} C \rightarrow$ 0 be a short exact sequence of $G$-modules that we also consider as a short exact sequence of $H$-modules. Then, for each $n \geq 0$, the corresponding diagram

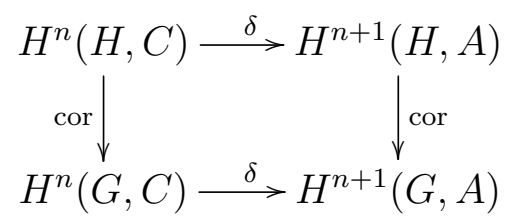

is commutative. The proof of this statement goes back to the explicit definition of $\delta$ that depends on the "snake lemma" (see [NSW00], p. 27, Prop. 1.3.3) and its proof). 
(3.2.9) If $H=G$, then $T=\{1\}$. In this case $\tilde{\sigma}=1$ for each $\sigma \in G$, so in the notation of $3.2 .2,{\widetilde{\sigma_{i}}}^{-1} \sigma_{i} \tau=\sigma_{i}$ for $i=1, \ldots, n$. Thus, in this case, $\operatorname{cor}(x)\left(\sigma_{1}, \ldots, \sigma_{n}\right)=x\left(\sigma_{1}, \ldots, \sigma_{n}\right)$, in other words cor is the identity map on $H^{n}(G, A)$.

Now, we come back to the case of number fields. Let $K / K_{0}$ be a finite Galois extension of number field and $A$ a $\operatorname{Gal}\left(K_{0}\right)$-module. Let $\mathfrak{p} \in \mathbb{P}\left(K_{0}\right)$. For each prime $\mathfrak{P} \in \mathbb{P}(K)$ lying over $\mathfrak{p}, A$ is a $\operatorname{Gal}\left(\hat{K}_{\mathfrak{P}}\right)$-module with $a^{\tau}=a^{\tau^{\lambda_{\mathfrak{P}}^{-1}}}$ for each $a \in A$ and $\tau \in \operatorname{Gal}\left(\hat{K}_{\mathfrak{P}}\right)$ (by the definition of $\lambda_{\mathfrak{P}}$ at the beginning of Section 1.2 if $\tau \in \operatorname{Gal}\left(\hat{K}_{\mathfrak{P}}\right)$, then $\left.\tau^{\lambda_{\mathfrak{P}}^{-1}} \in \operatorname{Gal}\left(K_{\mathfrak{P}}\right) \leq \operatorname{Gal}(K)\right)$.

We want to prove that the following diagram

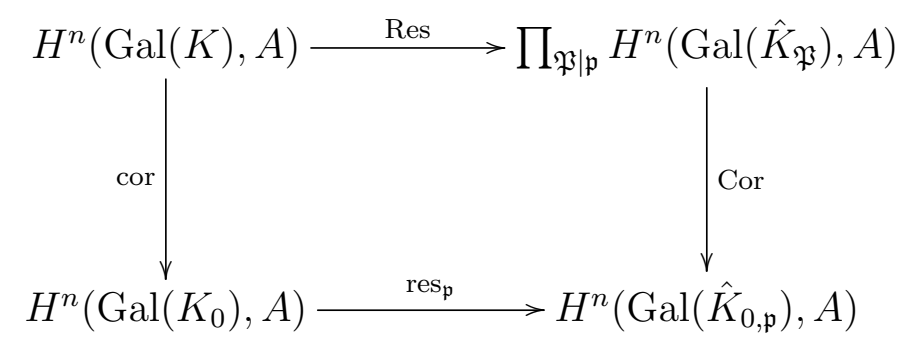

is commutative for each integer $n \geq 0$. In this diagram

(a) The map cor: $H^{n}(\mathrm{Gal})(K) \rightarrow H^{n}\left(\mathrm{Gal}\left(K_{0}\right), A\right)$ is the corestriction map defined below using that $\operatorname{Gal}(K)$ is an open subgroup of $\operatorname{Gal}\left(K_{0}\right)$.

(b) The map res: $H^{n}\left(\operatorname{Gal}\left(K_{0}\right), A\right) \rightarrow H^{n}\left(\operatorname{Gal}\left(\hat{K}_{0, \mathfrak{p}}\right), A\right)$ is the restriction map defined by $\operatorname{res}(u)=u_{\mathfrak{p}}$ where $u_{\mathfrak{p}}\left(\sigma_{0}, \ldots, \sigma_{n}\right)=u\left(\left.\sigma_{0}\right|_{\widetilde{K_{0}}}, \ldots,\left.\sigma_{n}\right|_{\widetilde{K_{0}}}\right)$ for each $u \in C^{n}\left(\operatorname{Gal}\left(K_{0}\right), A\right)$ and $\sigma_{0}, \ldots, \sigma_{n} \in \operatorname{Gal}\left(\hat{K}_{0, \mathfrak{p}}\right)$.

(c) The map Res is an abbreviation to the system of maps $\left(\text { res }_{\mathfrak{P}}\right)_{\mathfrak{P} \mid \mathfrak{p}}$ where $\operatorname{res}_{\mathfrak{P}}: H^{n}(\operatorname{Gal}(K), A) \rightarrow H^{n}\left(\operatorname{Gal}\left(\hat{K}_{\mathfrak{P}}\right), A\right)$ is the restriction map defined $\operatorname{res}_{\mathfrak{P}}(h)=h_{\mathfrak{P}}$ where $h_{\mathfrak{P}}\left(\sigma_{0}, \ldots, \sigma_{n}\right)=h\left({\sigma_{0}^{\lambda_{\mathfrak{P}}^{-1}}}^{-1} \ldots, \sigma_{n}^{\lambda_{\mathfrak{P}}^{-1}}\right)$ for each $h \in$ $C^{n}(\operatorname{Gal}(K), A)$ and $\sigma_{0}, \ldots, \sigma_{n} \in \operatorname{Gal}\left(\hat{K}_{\mathfrak{P}}\right)$.

(d) The map Cor is the product of the corestriction maps $\operatorname{cor}_{\mathfrak{P}}: H^{n}\left(\operatorname{Gal}\left(\hat{K}_{\mathfrak{P}}\right), A\right) \rightarrow$ $H^{n}\left(\operatorname{Gal}\left(\hat{K}_{0, \mathfrak{p}}\right), A\right)$ defined below, using that $\operatorname{Gal}\left(\hat{K}_{\mathfrak{P}}\right)$ is considered as an open subgroup of $\operatorname{Gal}\left(\hat{K}_{0, \mathfrak{p}}\right)$. 
Set $d=\left[K: K_{0}\right]$ and $d_{\mathfrak{P}}=\left[\hat{K}_{\mathfrak{P}}: \hat{K}_{0, \mathfrak{p}}\right]$ for $\mathfrak{P} \mid \mathfrak{p}$. Then, we write

$$
\operatorname{Gal}\left(K_{0}\right)=\bigcup_{j=1}^{d} \operatorname{Gal}(K) \varepsilon_{j}^{-1} \text { and } \operatorname{Gal}\left(\hat{K}_{0, \mathfrak{p}}\right)=\bigcup_{k=1}^{d_{\mathfrak{P}}} \operatorname{Gal}\left(\hat{K}_{\mathfrak{P}}\right) \varepsilon_{\mathfrak{P}, k}^{-1}
$$

Then, $f(X)=\prod_{j=1}^{d}\left(X-x^{\varepsilon_{j}^{-1}}\right)$ and $f_{\mathfrak{P}}(X)=\prod_{k=1}^{d_{\mathfrak{P}}}\left(X-x_{\mathfrak{P}}^{\varepsilon_{\mathfrak{P}, k}^{-1}}\right)$ for $\mathfrak{P} \mid \mathfrak{p}$. It follows that there exists a bijection of sets $\beta: \bigcup_{\mathfrak{P} \mid \mathfrak{p}}\left\{(\mathfrak{P}, 1), \ldots,\left(\mathfrak{P}, d_{\mathfrak{P}}\right)\right\} \longrightarrow$ $\{1, \ldots, d\}$ such that

$$
\left.\varepsilon_{\mathfrak{P}, k}^{-1}\right|_{K}=\left.\varepsilon_{\beta(\mathfrak{P}, k)}^{-1}\right|_{K} \text { for } i=1, \ldots, m \text { and } k=1, \ldots, d_{i} \text {. }
$$

Hence, there exists $\eta_{\mathfrak{P}, k} \in \operatorname{Gal}(K)$ such that

$$
\left.\varepsilon_{\mathfrak{P}, k}^{-1}\right|_{\widetilde{K_{0}}}=\eta_{\mathfrak{P}, k} \varepsilon_{\beta(\mathfrak{P}, k)}^{-1}
$$

For each $\sigma \in \operatorname{Gal}\left(K_{0}\right)$ we use 3.2.11) to choose $\tilde{\sigma} \in\left\{\varepsilon_{1}, \ldots, \varepsilon_{d}\right\}$ with $\operatorname{Gal}(K) \sigma^{-1}=\operatorname{Gal}(K) \tilde{\sigma}^{-1}$. Then

$$
\text { cor: } H^{n}(\operatorname{Gal}(K), A) \longrightarrow H^{n}\left(\operatorname{Gal}\left(K_{0}\right), A\right)
$$

is defined for each homogeneous cochain $h$ : $\operatorname{Gal}(K)^{n+1} \longrightarrow A$ and for all $\left(\sigma_{0}, \ldots, \sigma_{n}\right) \in \operatorname{Gal}\left(K_{0}\right)^{n+1}$ by

$$
\operatorname{cor}(h)\left(\sigma_{0}, \ldots, \sigma_{n}\right)=\prod_{j=1}^{d} h\left({\widetilde{\sigma_{0} \varepsilon_{j}}}^{-1} \sigma_{0} \varepsilon_{j}, \ldots,{\widetilde{\sigma_{n} \varepsilon_{j}}}^{-1} \sigma_{n} \varepsilon_{j}\right)^{\varepsilon_{j}^{-1}}
$$

Similarly for each $i$ between 1 and $m$ and every $\sigma \in \operatorname{Gal}\left(\hat{K}_{0, \mathfrak{p}}\right)$ there is a unique $\tilde{\sigma} \in\left\{\varepsilon_{\mathfrak{P}, 1}, \ldots, \varepsilon_{\mathfrak{P}, d_{\mathfrak{P}}}\right\}$ such that $\operatorname{Gal}\left(\hat{K}_{\mathfrak{P}}\right) \sigma^{-1}=\operatorname{Gal}\left(\hat{K}_{\mathfrak{P}}\right) \tilde{\sigma}^{-1}$. Again, we have for each homogeneous cochain $h$ : $\operatorname{Gal}\left(\hat{K}_{\mathfrak{P}}\right)^{n+1} \longrightarrow A$ and every tuple $\left(\sigma_{0}, \ldots, \sigma_{n}\right) \in \operatorname{Gal}\left(\hat{K}_{0, \mathfrak{p}}\right)^{n+1}$ that

$$
\operatorname{cor}_{\mathfrak{P}}(h)\left(\sigma_{0}, \ldots, \sigma_{n}\right)=\prod_{k=1}^{d_{\mathfrak{P}}} h\left({\widetilde{\sigma_{0} \varepsilon_{\mathfrak{P}, k}}}^{-1} \sigma_{0} \varepsilon_{i, k}, \ldots,{\widetilde{\sigma_{n} \varepsilon_{i, k}}}^{-1} \sigma_{n} \varepsilon_{\mathfrak{P}, k}\right)^{\varepsilon_{\mathfrak{P}, k}^{-1}}
$$

Lemma 3.2.1. Diagram 3.2.10 commutes for $n=0$ 
Proof. By (3.2.7),

$$
H^{0}(\operatorname{Gal}(K), A)=A^{\operatorname{Gal}(K)}=\left\{a \in A \mid a^{\sigma}=a \text { for all } \sigma \in \operatorname{Gal}(K)\right\}
$$

and the map cor: $H^{0}(\operatorname{Gal}(K), A) \longrightarrow H^{0}\left(\operatorname{Gal}\left(K_{0}\right), A\right)$ is defined (in terms of inhomogeneous 0-cochains) for each $a \in A^{\operatorname{Gal}(K)}$ by $\operatorname{cor}(a)=\prod_{j=1}^{d} a^{\varepsilon_{j}^{-1}}$ (see 3.2.14). Similarly, for each $\mathfrak{P} \mid \mathfrak{p}$ we have $H^{0}\left(\operatorname{Gal}\left(\hat{K}_{\mathfrak{P}}\right), A\right)=A^{\operatorname{Gal}\left(\hat{K}_{\mathfrak{P}}\right)}$ and cor $=\operatorname{cor}_{\mathfrak{P}}: H^{0}\left(\operatorname{Gal}\left(\hat{K}_{\mathfrak{P}}\right), A\right) \rightarrow H^{0}\left(\operatorname{Gal}\left(\hat{K}_{0, \mathfrak{p}}\right), A\right)$ is defined by $\operatorname{cor}(a)=$ $\prod_{k=1}^{d_{\mathfrak{P}}} a^{\varepsilon_{\mathfrak{P}, k}^{-1}}$. Furthermore, for each $a \in A^{\operatorname{Gal}(K)}$ and $\sigma \in \operatorname{Gal}\left(\hat{K}_{\mathfrak{P}}\right), a^{\sigma}=$ $a^{\sigma^{\lambda_{\mathfrak{P}}^{-1}}}=a$, so $\operatorname{res}_{\mathfrak{P}}(a)=a$. Similarly, if $a \in A^{\operatorname{Gal}\left(K_{0}\right)}$ and $\sigma \in \operatorname{Gal}\left(\hat{K}_{0, \mathfrak{p}}\right)$, $a^{\sigma}=a^{\left.\sigma\right|_{\tilde{K}_{0}}}=a$, so $\operatorname{res}(a)=a$.

Now, let $a \in A^{\operatorname{Gal}(K)}$. Then

$$
\begin{aligned}
\operatorname{Cor}(\operatorname{Res}(a)) & =\operatorname{Cor}(a, \ldots, a)=\prod_{\mathfrak{P} \mid \mathfrak{p}} \operatorname{cor}_{\mathfrak{P}}(a) \\
& =\prod_{\mathfrak{P} \mid \mathfrak{p}} \prod_{k=1}^{d_{\mathfrak{P}}} a^{\left.\varepsilon_{i, k}^{-1}\right|_{\bar{K}}}=\prod_{\mathfrak{P} \mid \mathfrak{p}} \prod_{k=1}^{d_{\mathfrak{P}}} a^{\eta_{\mathfrak{P}, k} \varepsilon_{\beta(\mathfrak{P}, k)}^{-1}}
\end{aligned}
$$

Since $\eta_{\mathfrak{P}, k} \in \operatorname{Gal}(K)$ (see 3.2.13) and $a \in A^{\operatorname{Gal}(K)}, a^{\eta_{\mathfrak{P}, k}}=a$, hence

$$
\begin{aligned}
\operatorname{Cor}(\operatorname{Res}(a)) & =\prod_{\mathfrak{P} \mid \mathfrak{p}} \prod_{k=1}^{d_{\mathfrak{P}}} a^{\varepsilon_{\beta(\mathfrak{P}, k)}^{-1}} \\
& =\prod_{j=1}^{d} a^{\varepsilon_{j}}=\operatorname{cor}(a)=\operatorname{cor}(\operatorname{res}(\mathrm{a})) .
\end{aligned}
$$

Thus, Cor $\circ$ Res $=$ res $\circ$ cor in dimension 0 .

The special case of the following result for $n=1$ is used in the proof of Theorem 1 of [Neu79] without a proof.

Proposition 3.2.2. The diagram 3.2 .10 commutes for every integer $n \geq 0$.

Proof. Each of the four vertices in diagram 3.2.10 can be considered as a cohomological functor in the sense of [Rib70] (p. 120, Def. 5.1). Moreover, 
res: $H^{n}(G, A) \longrightarrow H^{n}(H, A)$ commutes with the connecting homomorphism $\delta$ for every profinite group $G$, every closed subgroup $H$, and every finite $G$ module $A$ ([Rib70], p. 135). The same holds for the maps induced by the conjugations with the $\lambda_{\mathfrak{P}}$ 's ([NSW00], Prop. 1.5.4 and Prop. 1.6.2). Hence, both horizontal maps in digram (3.2.10) are morphisms of cohomological functors in the sense of Rib70] (p. 121, Def. 5.2). The same holds for both corestriction maps in $(3.2 .10)$, with $H$ now open in $G$ ([Rib70], p. 136). By Lemma 3.2.1, Cor $\circ$ Res $=$ res $\circ$ cor in dimension 0 . Hence, the method of dimension shifting of cohomology theory (in particular, Rib70, p. 124, Cor. 5.6) implies that the latter relation holds for each integer $n \geq 0$, as claimed.

\subsection{Bound on the Ramification of 1-Cocycles}

This section contains the main result of this chapter.

Let $K / K_{0}$ be a finite Galois extension of number fields, let $\mathfrak{p}$ be a prime of $K_{0}$, and let $\mathfrak{P}$ be a prime of $K$ that lies over $\mathfrak{p}$. The case $n=1$ of Proposition 3.2 .2 supplies a commutative diagram

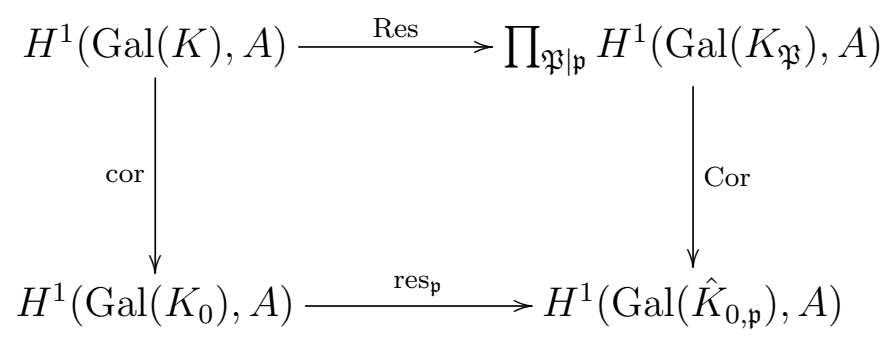

Now, suppose that $\operatorname{Gal}(K)$ acts trivially on $A$, so $H^{1}(\operatorname{Gal}(K), A)=\operatorname{Hom}(\operatorname{Gal}(K), A)$.

Then, $\operatorname{res}_{\mathfrak{P}}: H^{1}(\operatorname{Gal}(K), A) \longrightarrow H^{1}\left(\operatorname{Gal}\left(\hat{K}_{\mathfrak{P}}\right), A\right)$ is defined by $\operatorname{res}_{\mathfrak{P}}(h)(\sigma)=$ $h\left(\sigma_{\mathfrak{P}}^{\lambda_{\mathfrak{P}}^{-1}}\right)$ for each homomorphism $h: \operatorname{Gal}(K) \longrightarrow A$ and each $\sigma \in \operatorname{Gal}\left(\hat{K}_{\mathfrak{P}}\right)$. Recall that, as in Def. 1.2.4(b), the homomorphism $h: \operatorname{Gal}(K) \longrightarrow A$ is unramfied at $\mathfrak{P}$ if $\operatorname{res}_{\mathfrak{P}}(h)\left(\hat{I}_{\mathfrak{P}}\right)=1$. 
Let $T$ be a system of representatives for the left cosets of $\operatorname{Gal}\left(\hat{K}_{0, \mathfrak{p}}\right) \bmod$ ulo $\operatorname{Gal}\left(\hat{K}_{\mathfrak{P}}\right)$. As before, for each $\sigma \in \operatorname{Gal}\left(\hat{K}_{0, \mathfrak{p}}\right)$, we let $\tilde{\sigma}$ be the unique element of $T$ with $\tilde{\sigma} \operatorname{Gal}\left(\hat{K}_{\mathfrak{P}}\right)=\sigma \operatorname{Gal}\left(\hat{K}_{\mathfrak{P}}\right)$. By 3.2 .2 , the map

$$
\operatorname{cor}_{\mathfrak{P}}: C^{1}\left(\operatorname{Gal}\left(\hat{K}_{\mathfrak{P}}\right), A\right) \longrightarrow C^{1}\left(\operatorname{Gal}\left(\hat{K}_{0, \mathfrak{p}}\right), A\right)
$$

is defined for each $h_{\mathfrak{P}} \in C^{1}\left(\operatorname{Gal}\left(K_{\mathfrak{P}}\right), A\right)$ and for all $\sigma_{0}, \sigma_{1} \in \operatorname{Gal}\left(K_{0, \mathfrak{p}}\right)$ by

$$
\operatorname{cor}_{\mathfrak{P}}\left(h_{\mathfrak{P}}\right)\left(\sigma_{0}, \sigma_{1}\right)=\prod_{\tau \in T} h_{\mathfrak{P}}\left({\widetilde{\sigma_{0}}}^{-1} \sigma_{0} \tau,{\widetilde{\sigma_{1}}}^{-1} \sigma_{1} \tau\right)^{\tau^{-1}}
$$

Remark 3.3.1. If $\mathfrak{p}$ totally splits in $K$, the map

$$
\text { Cor: } \left.\prod_{\mathfrak{P} \mid \mathfrak{p}} H^{1}\left(\operatorname{Gal}\left(\hat{K}_{\mathfrak{P}}\right), A\right)\right)_{\mathfrak{P} \mid \mathfrak{p}} \longrightarrow H^{1}\left(\operatorname{Gal}\left(\hat{K}_{\mathfrak{p}}\right), A\right)
$$

is surjective. Indeed, $\operatorname{Gal}\left(\hat{K}_{\mathfrak{P}}\right)=\operatorname{Gal}\left(\hat{K}_{0, \mathfrak{p}}\right)$ for each $\mathfrak{P} \mid \mathfrak{p}$, so by $(3.2 .9)$, $\operatorname{cor}_{\mathfrak{P}}: H^{1}\left(\operatorname{Gal}\left(\hat{K}_{\mathfrak{P}}\right), A\right) \rightarrow H^{1}\left(\operatorname{Gal}\left(\hat{K}_{0, \mathfrak{p}}\right), A\right)$ is the identity map. Let $h_{\mathfrak{p}} \in$ $H^{1}\left(\operatorname{Gal}\left(\hat{K}_{0, \mathfrak{p}}\right), A\right)$ and let $\left(h_{\mathfrak{P}}\right)_{\mathfrak{P}} \in\left(H^{1}\left(\operatorname{Gal}\left(\hat{K}_{\mathfrak{P}}\right), A\right)\right)_{\mathfrak{P} \mid \mathfrak{p}}$ where $h_{\mathfrak{P}}=h_{\mathfrak{p}}$ for one $\mathfrak{P} \mid \mathfrak{p}$ and $h_{\mathfrak{P}^{\prime}}=1$ for each $\mathfrak{P}^{\prime} \neq \mathfrak{P}$. Then $\operatorname{Cor}\left(\left(h_{\mathfrak{P}}\right)_{\mathfrak{P}}\right)=\prod_{\mathfrak{P} \mid \mathfrak{p}} \operatorname{cor}_{\mathfrak{P}}\left(h_{\mathfrak{P}}\right)=$ $h_{\mathfrak{P}} \cdot 1 \cdots 1=h_{\mathfrak{P}}=h_{\mathfrak{p}}$.

Lemma 3.3.2. Suppose $\mathfrak{p} \in \mathbb{P}\left(K_{0}\right)$ is unramified in $K$. For each $\mathfrak{P} \in$ $\mathbb{P}(K)$ lying over $\mathfrak{p}$, let $h_{\mathfrak{P}} \in H^{1}\left(\operatorname{Gal}\left(\hat{K}_{\mathfrak{P}}\right), A\right)=\operatorname{Hom}\left(\operatorname{Gal}\left(\hat{K}_{\mathfrak{P}}\right), A\right)$ such that $h_{\mathfrak{P}}\left(\hat{I}_{\mathfrak{P}}\right)=1$. Let $u_{\mathfrak{p}}=\prod_{\mathfrak{P} \mid \mathfrak{p}} \operatorname{cor}_{\mathfrak{P}}\left(h_{\mathfrak{P}}\right)$. Then, $\left.u_{\mathfrak{p}}\right|_{\hat{I}_{\mathfrak{p}}}=1$.

Proof. For each $\mathfrak{P} \mid \mathfrak{p}$, we also denote by $h_{\mathfrak{P}} \in C^{1}\left(\operatorname{Gal}\left(\hat{K}_{\mathfrak{P}}\right), A\right)$ the homogeneous cochain that corresponds to $h_{\mathfrak{P}}($ see 1.4 .4$)$. Then, $\left.h_{\mathfrak{P}}\right|_{\hat{I}_{\mathfrak{P}} \times \hat{I}_{\mathfrak{P}}}=1$. Similarly, we denote $u_{\mathfrak{p}}$ as well the homogeneous cochain corresponding to $u_{\mathfrak{p}}$. Since $\mathfrak{p}$ is unramified in $K$, for each $\sigma \in \hat{I}_{\mathfrak{p}}$ we have $\sigma \in \hat{I}_{\mathfrak{P}}$ for each $\mathfrak{P} \mid \mathfrak{p}$, so $\hat{I}_{\mathfrak{P}}$ is a normal subgroup of $\operatorname{Gal}\left(\hat{K}_{0, \mathfrak{p}}\right)$. Let $\tau \in T_{\mathfrak{P}}$, where $T_{\mathfrak{P}}$ is a system of representatives of the left cosets of $\operatorname{Gal}\left(\hat{K}_{\mathfrak{p}}\right)$ modulo $\operatorname{Gal}\left(\hat{K}_{\mathfrak{P}}\right)$. Then, $\tau^{-1} \sigma \tau \in \hat{I}_{\mathfrak{P}}$. $\operatorname{Moreover,} \widetilde{\sigma \tau} \operatorname{Gal}\left(\hat{K}_{\mathfrak{P}}\right)=\sigma \tau \operatorname{Gal}\left(\hat{K}_{\mathfrak{P}}\right)=\tau\left(\tau^{-1} \sigma \tau\right) \operatorname{Gal}\left(\hat{K}_{\mathfrak{P}}\right)=\tau \operatorname{Gal}\left(\hat{K}_{\mathfrak{P}}\right)$. So 
$\widetilde{\sigma \tau}=\tau$ and $\widetilde{\sigma \tau}^{-1} \sigma \tau=\tau^{-1} \sigma \tau \in \hat{I}_{\mathfrak{P}}$. Thus for each $\sigma_{0}, \sigma_{1} \in \hat{I}_{\mathfrak{p}}$ we have by (3.2.2) that

$$
\begin{aligned}
\operatorname{cor}_{\mathfrak{P}}\left(h_{\mathfrak{P}}\right)\left(\sigma_{0}, \sigma_{1}\right) & =\prod_{\tau \in T_{\mathfrak{P}}} h_{\mathfrak{P}}\left({\widetilde{\sigma_{0} \tau}}^{-1} \sigma_{0} \tau,{\widetilde{\sigma_{1} \tau}}^{-1} \sigma_{1} \tau\right)^{\tau^{-1}} \\
& =\prod_{\tau \in T_{\mathfrak{P}}} h_{\mathfrak{P}}\left(\tau^{-1} \sigma_{0} \tau, \tau^{-1} \sigma_{1} \tau\right)^{\tau^{-1}}
\end{aligned}
$$

Since $\tau^{-1} \sigma_{i} \tau \in \hat{I}_{\mathfrak{P}}$ for $i=0,1$, we have $h_{\mathfrak{P}}\left(\tau^{-1} \sigma_{0} \tau, \tau^{-1} \sigma_{1} \tau\right)=1$. Hence $\operatorname{cor}_{\mathfrak{P}}\left(h_{\mathfrak{P}}\right)\left(\sigma_{0}, \sigma_{1}\right)=1$. It follows that

$$
u_{\mathfrak{p}}\left(\sigma_{0}, \sigma_{1}\right)=\prod_{\mathfrak{P} \mid \mathfrak{p}} \operatorname{cor}_{\mathfrak{P}}\left(h_{\mathfrak{P}}\right)\left(\sigma_{0}, \sigma_{1}\right)=1 \text { for } \sigma_{0}, \sigma_{1} \in \hat{I}_{\mathfrak{p}} .
$$

Corollary 3.3.3. If $A=C_{l, 1} \times \cdots \times C_{l, r}$ where each $C_{l, j}$ is an isomorphic copy of $C_{l}$, we fix an $1 \leq i \leq r$ and consider the prime $\mathfrak{q}_{i} \in \mathbb{P}\left(K_{0}\right)$ and the homomorphism $h$ : $\operatorname{Gal}(K) \rightarrow A$ given by Proposition 2.4.1. Let $u=$ $\operatorname{cor}(h) \in H^{1}\left(\operatorname{Gal}\left(K_{0}\right), A\right)$. Then $u_{\mathfrak{q}_{i}}=\operatorname{res}_{\mathfrak{q}_{i}}(u) \in H^{1}\left(\operatorname{Gal}\left(\hat{K}_{0, \mathfrak{q}_{i}}\right), A\right)$ is a cyclic (possibly trivial) homomorphism $u_{\mathfrak{q}_{i}}: \operatorname{Gal}\left(\hat{K}_{0, \mathfrak{q}_{i}}\right) \longrightarrow C_{l} \leq A$.

Proof. By (a) of Proposition 2.4.1, $\mathfrak{q}_{i}$ totally splits in $K$. Let $\mathfrak{Q}_{i, 1}, \ldots, \mathfrak{Q}_{i, k}$ be the primes of $K$ lying over $\mathfrak{q}_{i}$ and let $\mathfrak{Q}_{i}=\mathfrak{Q}_{i, 1}$ the prime given by (c) of Proposition 2.4.1. For each $t=1, \ldots, k, \operatorname{Gal}\left(\hat{K}_{\mathfrak{Q}_{i, t}}\right)=\operatorname{Gal}\left(\hat{K}_{0, \mathfrak{q}_{i}}\right)$, so $\operatorname{Gal}\left(\hat{K}_{0, q_{i}}\right)$ acts trivially on $A$. Therefore $u_{\mathfrak{q}_{i}}$ is a homomorphism. Let $h_{\mathfrak{Q}_{i, t}}=\operatorname{res}_{\mathfrak{Q}_{i, t}}(h)$ for each $t=1, \ldots, k$. By the diagram 3.3.1), $u_{\mathfrak{q}_{i}}=$ $\prod_{t=1}^{k} \operatorname{cor}_{\mathfrak{Q}_{i, t}}\left(h_{\mathfrak{Q}_{i, t}}\right)$. Since, $\operatorname{Gal}\left(\hat{K}_{\mathfrak{Q}_{i, t}}\right)=\operatorname{Gal}\left(\hat{K}_{0, \mathfrak{q}_{i}}\right)$, the map $\operatorname{cor}_{\mathfrak{Q}_{i, t}}$ is the identity map for each $t=1, \ldots, k$. It follows that $u_{\mathfrak{q}_{i}}=\prod_{t=1}^{k} h_{\mathfrak{Q}_{i, t}}$. By (c) and (d) of Proposition 2.4.1. $\operatorname{Im}\left(h_{\mathfrak{Q}_{i, 1}}\right)=1 \times \cdots \times 1 \times C_{l, i} \times 1 \times \cdots \times 1$ and $\operatorname{Im}\left(h_{\mathfrak{Q}_{i, t}}\right) \leq 1 \times \cdots \times 1 \times C_{l, i} \times 1 \times \cdots \times 1$ for each $t=2, \ldots, k$. It follows that $\operatorname{Im}\left(\prod_{t=1}^{k} h_{\mathfrak{Q}_{i, t}}\right) \leq 1 \times \cdots \times 1 \times C_{l, i} \times 1 \times \cdots \times 1$. Therefore, $u_{\mathfrak{q}_{i}}$ is cyclic homomorphism.

The following local-global result of Neukirch is an important tool for the construction of our desired element $x \in H^{1}\left(\operatorname{Gal}\left(K_{0}\right), A\right)$ described in the introduction of this chapter. 
Lemma 3.3.4 ([Neu79], Lem. 3). Let $A=C_{l}^{r}$ be a simple Gal $\left(K_{0}\right)$-module on which $\operatorname{Gal}(K)$ acts trivially. Suppose $\zeta_{l} \notin K$. Let $T$ be a finite set of primes of $K_{0}$ and for each $\mathfrak{p} \in T$ consider an element $y_{\mathfrak{p}} \in H^{1}\left(\operatorname{Gal}\left(\hat{K}_{0, \mathfrak{p}}\right), A\right)$. Then there exists an element $z \in H^{1}\left(\operatorname{Gal}\left(K_{0}\right), A\right)$ such that, if we denote $z_{\mathfrak{p}}=\operatorname{res}_{\mathfrak{p}}(z)$, then

(a) for each $\mathfrak{p} \in T, z_{\mathfrak{p}}=y_{\mathfrak{p}}$,

(b) if $\mathfrak{p} \in \mathbb{P}\left(K_{0}\right) \backslash T$ and $z_{\mathfrak{p}}$ is ramified, then $\mathfrak{p}$ totally splits in $K\left(\zeta_{l}\right)$.

We come now to the proof of the main result of this chapter. The first step in the construction of the element $x \in H^{1}\left(\mathrm{Gal}\left(K_{0}\right), A\right)$ changes each given element $y_{\mathfrak{p}} \in H^{1}\left(\operatorname{Gal}\left(\hat{K}_{0, \mathfrak{p}}\right), A\right)$ to an element $\eta_{\mathfrak{p}} \in H^{1}\left(\operatorname{Gal}\left(\hat{K}_{0, \mathfrak{p}}\right), A\right)$ that belongs to the image of the corestriction map. After that, we shift the $\eta_{\mathfrak{p}}$ 's to elements of $H^{1}\left(\operatorname{Gal}\left(\hat{K}_{\mathfrak{P}}\right), A\right)$ and use Proposition 2.4.1 to find a homomorphism $h$ : $\operatorname{Gal}(K) \longrightarrow A$ with a bound on its ramification. The corestriction map maps $h$ onto an element $u \in H^{1}\left(\operatorname{Gal}\left(K_{0}\right), A\right)$ which when multiplied by an element $z \in H^{1}\left(\operatorname{Gal}\left(K_{0}\right), A\right)$ from Lemma 3.3.4 gives the desired element $x$.

Proposition 3.3.5. Let $K_{0} \subseteq K \subseteq L$ a tower of finite Galois extension of number fields such that $L / K$ is an abelian $l$-extension. Suppose $\zeta_{l} \notin K$. Let $A=C_{l}^{r}$ be a simple $\operatorname{Gal}\left(K_{0}\right)$-module on which $\operatorname{Gal}(K)$ acts trivially. Let $n$ be a multiple of $l$ and let $T$ be a finite set of primes of $K_{0}$ that contains all primes which are ramified in $K$. Let $T_{K}$ be the set of primes of $K$ that divide the primes in $T$. Suppose $S_{0} \subseteq T_{K}$. For each $\mathfrak{p} \in T$, let $y_{\mathfrak{p}} \in H^{1}\left(\operatorname{Gal}\left(\hat{K}_{0, \mathfrak{p}}\right), A\right)$. Then there exist $\mathfrak{q}_{1}, \ldots, \mathfrak{q}_{r} \in \mathbb{P}\left(K_{0}\right) \backslash T$ and there exists an element $x \in H^{1}\left(\operatorname{Gal}\left(K_{0}\right), A\right)$ such that, if $x_{\mathfrak{p}}=\operatorname{res}_{\mathfrak{p}}(x)$ then

(a) for each $\mathfrak{p} \in T, x_{\mathfrak{p}}=y_{\mathfrak{p}}$,

(b) for each $\mathfrak{p} \in \mathbb{P}\left(K_{0}\right) \backslash\left(T \cup\left\{\mathfrak{q}_{1}, \ldots, \mathfrak{q}_{r}\right\}\right)$, the element $x_{\mathfrak{p}}$ is unramified, and

(c) for $i=1, \ldots, r$, the prime $\mathfrak{q}_{i}$ totally splits in $L\left(\zeta_{n}\right)$, and $x_{\mathfrak{q}_{i}}: \operatorname{Gal}\left(\hat{K}_{0, \mathfrak{q}_{i}}\right) \rightarrow$ $C_{l} \leq A$ is a cyclic homomorphism. 
(d) Let $G$ and $\bar{G}$ be finite groups with $A \leq \bar{G}, \lambda: G \rightarrow \bar{G}$ an epimorphism. Suppose that $|\operatorname{Ker}(\lambda)| \cdot l$ divides $n$. Then, for each $i=1, \ldots, r$ there exists a homomorphism $x_{\mathfrak{q}_{i}}^{\prime}: \operatorname{Gal}\left(\hat{K}_{0, \mathfrak{q}_{i}}\right) \rightarrow G$ such that $\lambda \circ x_{\mathfrak{q}_{i}}^{\prime}=x_{\mathfrak{q}_{i}}$.

Proof. By Lemma 3.3.4 there exists an element $z \in H^{1}\left(\operatorname{Gal}\left(K_{0}\right), A\right)$ such that

(3.3.4) for each $\mathfrak{p} \in T, z_{\mathfrak{p}}=y_{\mathfrak{p}}$,

(3.3.5) if $\mathfrak{p} \in \mathbb{P}\left(K_{0}\right) \backslash T$ and $z_{\mathfrak{p}}$ is ramified, then $\mathfrak{p}$ totally splits in $K\left(\zeta_{l}\right)$, in particular in $K$.

We divide the rest of the proof in three parts.

Part A: Definition of $\eta_{\mathfrak{p}}$. Let $V=T \cup\left\{\mathfrak{p} \in \mathbb{P}\left(K_{0}\right) \mid z_{\mathfrak{p}}\right.$ is ramified $\}$. For each $\mathfrak{p} \in V$ we define $\eta_{\mathfrak{p}} \in H^{1}\left(\operatorname{Gal}\left(\hat{K}_{0, \mathfrak{p}}\right), A\right)$ by

$$
\begin{aligned}
& \eta_{\mathfrak{p}}=1 \text { for } \mathfrak{p} \in T \\
& \eta_{\mathfrak{p}}=z_{\mathfrak{p}}^{-1} \text { for } \mathfrak{p} \in V \backslash T
\end{aligned}
$$

Claim: For each $\mathfrak{p} \in V$, the element $\eta_{\mathfrak{p}} \in H^{1}\left(\operatorname{Gal}\left(\hat{K}_{0, \mathfrak{p}}\right), A\right)$ lies in the image of

$$
\text { Cor: } \prod_{\mathfrak{P} \mid \mathfrak{p}} H^{1}\left(\operatorname{Gal}\left(\hat{K}_{\mathfrak{P}}\right), A\right) \longrightarrow H^{1}\left(\operatorname{Gal}\left(\hat{K}_{0, \mathfrak{p}}\right), A\right)
$$

Indeed, the statement holds for $\mathfrak{p} \in T$ since, by $(3.3 .6), \eta_{\mathfrak{p}}=1$ and since Cor $=\prod_{\mathfrak{P} \mid \mathfrak{p}} \operatorname{cor}_{\mathfrak{P}}$ is a homomorphism of groups. If $\mathfrak{p} \in V \backslash T$, then $z_{\mathfrak{p}}$ is ramified, so by (3.3.5), $\mathfrak{p}$ totally splits in $K$. It follows from Remark 3.3.1 that the map in 3.3.7 is surjective, so $\eta_{\mathfrak{p}}$ belongs to the image of Cor as claimed.

Part B: Shifting the $\eta_{\mathfrak{p}}$ 's. For each $\mathfrak{p} \in V$ we choose a pre-image $\left(\tilde{\eta}_{\mathfrak{P}}\right)_{\mathfrak{P} \mid \mathfrak{p}} \in$ $\prod_{\mathfrak{P} \mid \mathfrak{p}} H^{1}\left(\operatorname{Gal}\left(\hat{K}_{\mathfrak{P}}\right), A\right)$ of $\eta_{\mathfrak{p}} \in H^{1}\left(\operatorname{Gal}\left(\hat{K}_{0, \mathfrak{p}}\right), A\right)$, that is

$$
\eta_{\mathfrak{p}}=\prod_{\mathfrak{P} \mid \mathfrak{p}} \operatorname{cor}_{\mathfrak{P}}\left(\tilde{\eta}_{\mathfrak{P}}\right), \quad \mathfrak{p} \in V
$$


Let $V_{K}=\{\mathfrak{P} \in \mathbb{P}(K)|\mathfrak{P}| \mathfrak{p}$ for a prime $\mathfrak{p} \in V\}$. Since $\operatorname{Gal}(K)$ acts trivially on $A$, the map $\tilde{\eta}_{\mathfrak{P}}: \operatorname{Gal}\left(\hat{K}_{\mathfrak{P}}\right) \longrightarrow A$ is a homomorphism for each $\mathfrak{P} \in V_{K}$. Likewise,

$$
z^{\prime}=\left.z\right|_{\operatorname{Gal}(K)}: \operatorname{Gal}(K) \longrightarrow A
$$

is a homomorphism. Let $L^{\prime}$ be the fixed field of $\operatorname{Ker}\left(z^{\prime}\right)$ in $\tilde{K}$. Then $L^{\prime}$ is a finite abelian $l$-extension of $K$, hence so is $L L^{\prime}$. Hence the Galois closure $L^{\prime \prime}$ of $L L^{\prime}$ over $K_{0}$ is a finite abelian $l$-extension of $K$. Considering the tower of number fields $K_{0} \subseteq K \subseteq L^{\prime \prime}$, the set of primes $V_{K}$, and the system of homomorphisms $\left(\tilde{\eta}_{\mathfrak{P}}\right)_{\mathfrak{P} \in V_{K}}$, Proposition 2.4.1, gives primes $\mathfrak{q}_{1}, \ldots, \mathfrak{q}_{r} \in$ $\mathbb{P}\left(K_{0}\right) \backslash V$ and a homomorphism $h: \operatorname{Gal}(K) \longrightarrow A$ such that, if we denote $h_{\mathfrak{P}}=\operatorname{res}_{\mathfrak{P}}(h)$, then

(3.3.10) $\mathfrak{q}_{i}$ totally splits in $L^{\prime \prime}\left(\zeta_{n}\right)$ for $i=1, \ldots, r$,

(3.3.11) $h_{\mathfrak{P}}=\tilde{\eta}_{\mathfrak{P}}$, for each $\mathfrak{P} \in V_{K}$,

(3.3.12) for $i=1, \ldots, r$ there exists $\mathfrak{Q}_{i} \in \mathbb{P}(K)$ with $\left.\mathfrak{Q}_{i}\right|_{K_{0}}=\mathfrak{q}_{i}$ such that $h\left(\operatorname{Gal}\left(\hat{K}_{\mathfrak{Q}_{i}}\right)\right) \cong C_{l}$,

$h_{\mathfrak{P}}\left(\hat{I}_{\mathfrak{P}}\right)=1$ for each $\mathfrak{P} \in \mathbb{P}(K) \backslash\left(V_{K} \cup\left\{\mathfrak{Q}_{1}, \ldots, \mathfrak{Q}_{r}\right\}\right)$,

We consider $u=\operatorname{cor}(h) \in H^{1}\left(\operatorname{Gal}\left(K_{0}\right), A\right)$ and set $u_{\mathfrak{p}}=\operatorname{res}_{\mathfrak{p}}(u)$ for each $\mathfrak{p} \in \mathbb{P}\left(K_{0}\right)$. By the commutativity of the diagram (3.3.1), by (3.3.11), and by $(3.3 .8)$ we have

$$
\begin{aligned}
u_{\mathfrak{p}} & =\operatorname{res}_{\mathfrak{p}}(\operatorname{cor}(h))=\operatorname{Cor}(\operatorname{Res}(h)) \\
& =\prod_{\mathfrak{P} \mid \mathfrak{p}} \operatorname{cor}_{\mathfrak{P}}\left(h_{\mathfrak{P}}\right)=\prod_{\mathfrak{P} \mid \mathfrak{p}} \operatorname{cor}_{\mathfrak{P}}\left(\tilde{\eta}_{\mathfrak{P}}\right)=\eta_{\mathfrak{p}}, \quad \mathfrak{p} \in V .
\end{aligned}
$$

Furthermore, by Corollary 3.3 .3 , for each $i=1, \ldots, r$

$$
\begin{aligned}
& u_{\mathfrak{q}_{i}}=\prod_{\mathfrak{Q} \mid \mathfrak{q}_{i}} \operatorname{cor}_{\mathfrak{Q}}\left(h_{\mathfrak{Q}}\right) \text { is a cyclic (possibly trivial) homomorphism } \\
& u_{\mathfrak{q}_{i}}: \operatorname{Gal}\left(\hat{K}_{0, \mathfrak{q}_{i}}\right) \longrightarrow C_{l}
\end{aligned}
$$


Part C: The element $x$. We prove that the element $x=u z \in H^{1}\left(\operatorname{Gal}\left(K_{0}\right), A\right)$ satisfies Conditions (a)-(d) of the Proposition.

Proof of (a): For each $\mathfrak{p} \in T$, we have from 3.3.13, (3.3.4), and 3.3.6 that

$$
x_{\mathfrak{p}}=u_{\mathfrak{p}} z_{\mathfrak{p}}=\eta_{\mathfrak{p}} y_{\mathfrak{p}}=1 y_{\mathfrak{p}}=y_{\mathfrak{p}} .
$$

Hence, $x$ satisfies Condition (a).

Proof of $(\mathrm{b}):$ Let $\mathfrak{p} \in \mathbb{P}\left(K_{0}\right) \backslash\left(T \cup\left\{\mathfrak{q}_{1}, \ldots, \mathfrak{q}_{r}\right\}\right)$. If $\mathfrak{p} \in V$, then, by 3.3.13 and 3.3.6

$$
x_{\mathfrak{p}}=u_{\mathfrak{p}} z_{\mathfrak{p}}=\eta_{\mathfrak{p}} z_{\mathfrak{p}}=z_{\mathfrak{p}}^{-1} z_{\mathfrak{p}}=1 .
$$

Hence, $x_{\mathfrak{p}}$ is unramified (Def. 3.1.2(a)). If $\mathfrak{p} \notin V$, then by the definition of $V$ in Part A, $z_{\mathfrak{p}}$ is unramified. Furthermore, since all ramified primes of $K / K_{0}$ are contained in $T \subseteq V, \mathfrak{p}$ is unramified in $K$. Since, by (3.3.12), $h_{\mathfrak{P}}\left(\hat{I}_{\mathfrak{P}}\right)=1$ for $\mathfrak{P} \mid \mathfrak{p}$, and since $u_{\mathfrak{p}}=\prod_{\mathfrak{P} \mid \mathfrak{p}} \operatorname{cor}_{\mathfrak{P}}\left(h_{\mathfrak{P}}\right)$ (by $(3.3 .13)$ ), it follows from Lemma 3.3 .2 that $\left.u_{\mathfrak{p}}\right|_{\hat{I}_{\mathfrak{p}}}=1$. Hence, $\left.x_{\mathfrak{p}}\right|_{\hat{I}_{\mathfrak{p}}}=\left.\left.u_{\mathfrak{p}}\right|_{\hat{I}_{\mathfrak{p}}} z_{\mathfrak{p}}\right|_{\hat{I}_{\mathfrak{p}}}=1$, so $x_{\mathfrak{p}}$ is unramified. Thus, $x$ satisfies (b).

Proof of (c): Consider $i$ between 1 and $r$. By (3.3.10), $\mathfrak{q}_{i}$ totally splits $K$. On one hand,

$$
u_{\mathfrak{q}_{i}}=\operatorname{res}_{\mathfrak{q}_{i}}(\operatorname{cor}(h)) .
$$

Hence by (3.3.14), $u_{\mathfrak{q}_{i}}: \operatorname{Gal}\left(\hat{K}_{0, \mathfrak{q}_{i}}\right) \longrightarrow C_{l} \leq A$ is a cyclic homomorphism. On the other hand, since $\operatorname{Gal}\left(\hat{K}_{0, \mathfrak{q}_{i}}\right)=\operatorname{Gal}\left(\hat{K}_{\mathfrak{Q}_{i, j}}\right)$ and since $\operatorname{Gal}(K)$ acts trivially on $A$, the group $\operatorname{Gal}\left(\hat{K}_{0, \mathfrak{q}_{i}}\right)$ acts trivially on $A$, so $z_{\mathfrak{q}_{i}} \in H^{1}\left(\operatorname{Gal}\left(\hat{K}_{0, \mathfrak{q}_{i}}\right), A\right)$ is a homomorphism and there exists $\mathfrak{Q}_{i, j} \mid \mathfrak{q}_{i}$ such that $z_{\mathfrak{q}_{i}}=\operatorname{res}_{\mathfrak{Q}_{i, j}}\left(z^{\prime}\right): \operatorname{Gal}\left(\hat{K}_{\mathfrak{Q}_{i, j}}\right) \longrightarrow$ $A$ with $z^{\prime}$ as in $(3.3 .9)$. Again by (3.3.10), $\mathfrak{Q}_{i, j}$ totally splits in $L^{\prime}$. Since $L^{\prime}$ is the fixed field of $\operatorname{Ker}\left(z^{\prime}\right)$ (Part B), it follows that $z_{\mathfrak{q}_{i}}$ is the trivial homomorphism. Then $x_{\mathfrak{q}_{i}}=u_{\mathfrak{q}_{i}}$, so it is cyclic homomorphism $x_{\mathfrak{q}_{i}}: \operatorname{Gal}\left(\hat{K}_{0, \mathfrak{q}_{i}}\right) \longrightarrow C_{l}$. Hence $x$ satisfies (c), as desired.

Proof of (d): We fix $i$ between 1 and $r$. By Condition (c), $x_{\mathfrak{q}_{i}}: \operatorname{Gal}\left(K_{0, \mathfrak{q}_{i}}\right) \rightarrow C_{l}$ 
is a cyclic homomorphism. If $x_{\mathfrak{q}_{i}}$ is unramified, then by Lemma 1.2.6, there exists a homomorphism $x_{\mathfrak{q}_{i}}^{\prime}: \operatorname{Gal}\left(K_{0, \mathfrak{q}_{i}}\right) \longrightarrow G$ such that $\lambda \circ x_{\mathfrak{q}_{i}}^{\prime}=x_{\mathfrak{q}_{i}}$. Suppose $x_{\mathfrak{q}_{i}}$ ramifies. Again by Condition (c), $\zeta_{n} \in \hat{K}_{0, \mathfrak{q}_{i}}$. Since $\mathfrak{q}_{i} \in \mathbb{P}\left(K_{0}\right) \backslash T$, $\mathfrak{q}_{i} \nmid l$, then the result follows from Lemma 1.2.7. 


\section{Chapter 4}

\section{Solving embedding problems with bounded ramification}

This chapter contains the main results of this work. Given a finite Galois extension $K / K_{0}$ of number fields, an epimorphism $\alpha: G \rightarrow \operatorname{Gal}\left(K / K_{0}\right)$ with a solvable kernel such that each of the corresponding local embedding problem is solvable, a finite subset $T$ of $\mathbb{P}\left(K_{0}\right)$ that contains $\operatorname{Ram}\left(K / K_{0}\right)$, and a local solution $\varphi_{\mathfrak{p}}: \operatorname{Gal}\left(\hat{K}_{0, \mathfrak{p}}\right) \rightarrow G$ for each $\mathfrak{p} \in T$, there exist, under certain conditions on the roots of unity in $K$, a proper solution $\psi: \operatorname{Gal}\left(K_{0}\right) \rightarrow G$ of the corresponding embedding problem and a set $R \subseteq \mathbb{P}\left(K_{0}\right) \backslash T$ such $\psi$ coincides with $\varphi_{\mathfrak{p}}$ for each $\mathfrak{p} \in T$ (i.e. for $\mathfrak{p} \in T, \psi$ defines the same local solution field as the solution field defined by $\left.\varphi_{\mathfrak{p}}\right)$ and $\operatorname{Ram}(\psi) \subseteq T \cup R$, and $|R|=\Omega(|\operatorname{Ker}(\alpha)|)$, where $\Omega$ is a well known arithmetical function (defined in (4.2.1). We start with the solution of the first layer, i.e. an embedding problem whose kernel is a simple $\operatorname{Gal}\left(K_{0}\right)$-module. Then, we use induction on the order of $\operatorname{Ker}(\alpha)$. In the last section of this chapter, we give some applications of the main result. We recall that every given homomorphism of a profinite group is assumed to be continuous.

For the rest of this chapter, as in Definition 1.2.1, if $\psi: \operatorname{Gal}\left(K_{0}\right) \rightarrow G$ is a homomorphism, then we denote $\psi_{\mathfrak{p}}: \operatorname{Gal}\left(\hat{K}_{0, \mathfrak{p}}\right) \rightarrow G$ the local restriction 
defined by $\psi_{\mathfrak{p}}(\sigma)=\psi\left(\left.\sigma\right|_{\tilde{K}_{0}}\right)$.

\subsection{The Principal Homogeneous Space over $H^{1}\left(\operatorname{Gal}\left(K_{0}\right), A\right)$}

As mentioned in the previous chapters, in order to carry out the induction step, the solution of the first layer must satisfy certain conditions. For that, we multiply the solution obtained by the local-global principle (Lemma 1.2.8) by a crossed homomorphism. This multiplication gives rise to an action of the first cohomology group $H^{1}\left(\operatorname{Gal}\left(K_{0}\right), A\right)$ on the set of the solutions of the embedding problem up to equivalence class (Definition 1.2.3), where $A$ is the kernel of the embedding problem which is abelian. In this section, we define this action and determine some of its properties for the special case $A=C_{l}^{r}$.

Let $K / K_{0}$ be a finite Galois extension of number fields with $\operatorname{Gal}\left(K / K_{0}\right)=\Gamma$. Let $\bar{G}$ be a finite group, and let $\bar{\alpha}: \bar{G} \longrightarrow \Gamma$ be an epimorphism. Consider the following embedding problem

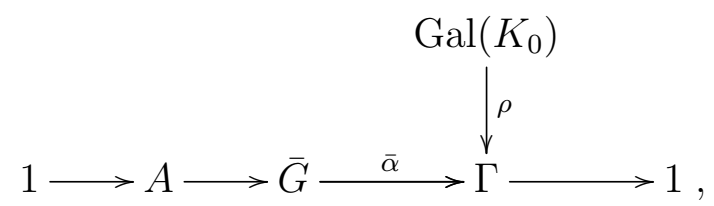

(4.1.2) where $\rho=\operatorname{res}_{\tilde{K}_{0} / K}$ and $A$ is a simple $\Gamma$-module with $A=C_{l}^{r}$, and $l$ is a prime number such that $\zeta_{l}$ is not fixed by $\operatorname{Ker}(\rho)$ (i.e. $\zeta_{l} \notin K$ ).

(4.1.3) The action of $\Gamma$ on $A$ is defined by

$$
\begin{aligned}
\Gamma \times A & \longrightarrow A \\
(\bar{g}, a) & \longmapsto a^{\bar{g}}=g^{-1} a g,
\end{aligned}
$$

where $g \in \bar{G}$ with $\bar{\alpha}(g)=\bar{g}$. The group $A$ becomes a simple $\operatorname{Gal}\left(K_{0}\right)$ module via $\rho$. If $\sigma \in \operatorname{Gal}\left(K_{0}\right)$ and $a \in A$, then $a^{\sigma}=a^{\rho(\sigma)}$. In particular, $\operatorname{Gal}(K)=\operatorname{Ker}(\rho)$ acts trivially on $A$. 
We recall that $\mathcal{H}_{\Gamma, \rho, \bar{\alpha}}\left(\operatorname{Gal}\left(K_{0}\right), \bar{G}\right)$ is the set of equivalence classes of the solutions of 4.1.1) and $\mathcal{H} \mathrm{om}_{\Gamma, \rho, \bar{\alpha}}\left(\operatorname{Gal}\left(K_{0}\right), \bar{G}\right)_{\text {sur }}$ is the subset of equivalence classes of the proper solutions (i.e. surjective solutions).

Lemma 4.1.1. Let $\psi: \operatorname{Gal}\left(K_{0}\right) \longrightarrow \bar{G}$ be a homomorphism such that $\bar{\alpha} \circ \psi=\rho$, and $\chi: \operatorname{Gal}\left(K_{0}\right) \longrightarrow A$ is a crossed homomorphism. Then $\psi \cdot \chi: \operatorname{Gal}\left(K_{0}\right) \longrightarrow \bar{G}$, defined by $(\psi \cdot \chi)(\sigma)=\psi(\sigma) \cdot \chi(\sigma)$ for $\sigma \in \operatorname{Gal}\left(K_{0}\right)$, is a homomorphism satisfying $\bar{\alpha} \circ(\psi \cdot \chi)=\rho$.

Proof. Denote $\psi^{\prime}=\psi \cdot \chi$. For all $\sigma_{1}, \sigma_{2} \in \operatorname{Gal}\left(K_{0}\right)$, we have

$$
\begin{aligned}
\psi^{\prime}\left(\sigma_{1} \sigma_{2}\right) & =\psi\left(\sigma_{1} \sigma_{2}\right) \chi\left(\sigma_{1} \sigma_{2}\right) \\
& =\psi\left(\sigma_{1}\right) \psi\left(\sigma_{2}\right) \chi\left(\sigma_{1}\right)^{\sigma_{2}} \chi\left(\sigma_{2}\right)
\end{aligned}
$$

Since $\bar{\alpha}\left(\psi\left(\sigma_{2}\right)\right)=\rho\left(\sigma_{2}\right)$, we have $\chi\left(\sigma_{1}\right)^{\sigma_{2}}=\chi\left(\sigma_{1}\right)^{\rho\left(\sigma_{2}\right)}=\psi\left(\sigma_{2}\right)^{-1} \chi\left(\sigma_{1}\right) \psi\left(\sigma_{2}\right)$. Hence,

$$
\begin{aligned}
\psi^{\prime}\left(\sigma_{1} \sigma_{2}\right) & =\psi\left(\sigma_{1}\right) \psi\left(\sigma_{2}\right) \psi\left(\sigma_{2}\right)^{-1} \chi\left(\sigma_{1}\right) \psi\left(\sigma_{2}\right) \chi\left(\sigma_{2}\right) \\
& =\psi\left(\sigma_{1}\right) \chi\left(\sigma_{1}\right) \psi\left(\sigma_{2}\right) \chi\left(\sigma_{2}\right) \\
& =\psi^{\prime}\left(\sigma_{1}\right) \psi^{\prime}\left(\sigma_{2}\right) .
\end{aligned}
$$

Moreover, for $\sigma \in \operatorname{Gal}\left(K_{0}\right), \bar{\alpha}\left(\psi^{\prime}(\sigma)\right)=\bar{\alpha}(\psi(\sigma)) \bar{\alpha}(\chi(\sigma))$. Since $\chi(\sigma) \in A=$ $\operatorname{Ker}(\bar{\alpha})$, we have $\bar{\alpha}\left(\psi^{\prime}(\sigma)\right)=\bar{\alpha}(\psi(\sigma))=\rho(\sigma)$, as desired.

The product defined in Lemma 4.1.1 gives rise to an action of $H^{1}\left(\operatorname{Gal}\left(K_{0}\right), A\right)$ on $\mathcal{H} \operatorname{om}_{\Gamma, \rho, \bar{\alpha}}\left(\operatorname{Gal}\left(K_{0}\right), \bar{G}\right)$. If $[\psi] \in \mathcal{H} \operatorname{om}_{\Gamma, \rho, \bar{\alpha}}\left(\operatorname{Gal}\left(K_{0}\right), \bar{G}\right)$ and $x \in H^{1}\left(\operatorname{Gal}\left(K_{0}\right), A\right)$, we define $\left[\psi^{\prime}\right]=[\psi]^{x}$ such that if $\psi: \operatorname{Gal}\left(K_{0}\right) \longrightarrow \bar{G}$ (resp. $\chi: \operatorname{Gal}\left(K_{0}\right) \longrightarrow A$ ) is a representative of $[\psi]$ (resp. of $x$ ), then $\psi^{\prime}=\psi \cdot \chi$ is a representative of $\left[\psi^{\prime}\right]$. In particular, $[\psi]^{x_{1} x_{2}}=\left[\psi \chi_{1} \chi_{2}\right]=\left([\psi]^{x_{1}}\right)^{x_{2}}$. This action does not depend on the choice of the representatives of $[\psi]$ and $x$. Indeed, let $\psi_{1}: \operatorname{Gal}\left(K_{0}\right) \longrightarrow \bar{G}$ be another representative of $[\psi]$. Then there exists $a \in A$ such that $\psi_{1}(\sigma)=a^{-1} \psi(\sigma) a$ for all $\sigma \in \operatorname{Gal}\left(K_{0}\right)$. If $\chi_{1}: \operatorname{Gal}\left(K_{0}\right) \longrightarrow A$ is another representative of $x$, then there exists a coboundary $\gamma: \operatorname{Gal}\left(K_{0}\right) \longrightarrow A$ such that $\chi_{1}=\gamma \chi$. Thus, there exists $b \in A$ with $\gamma(\sigma)=b^{\sigma} b^{-1}$ for all 
$\sigma \in \operatorname{Gal}\left(K_{0}\right)$. Now let $\psi^{\prime \prime}=\psi_{1} \cdot \chi_{1}$. For $\sigma \in \operatorname{Gal}\left(K_{0}\right)$, we have, since $A$ is abelian

$$
\begin{aligned}
\psi^{\prime \prime}(\sigma) & =\psi_{1}(\sigma) \cdot \chi_{1}(\sigma)=a^{-1} \psi(\sigma) a \cdot \gamma(\sigma) \chi(\sigma) \\
& =a^{-1} \psi(\sigma) a \cdot b^{\sigma} b^{-1} \chi(\sigma) \\
& =a^{-1} \psi(\sigma) b^{\sigma} a b^{-1} \chi(\sigma) .
\end{aligned}
$$

Now, $b^{\sigma}=b^{\rho(\sigma)}$. Since $\bar{\alpha}(\psi(\sigma))=\rho(\sigma), b^{\rho(\sigma)}=\psi(\sigma)^{-1} b \psi(\sigma)$. Thus,

$$
\begin{aligned}
\psi^{\prime \prime}(\sigma) & =a^{-1} \psi(\sigma) \psi(\sigma)^{-1} b \psi(\sigma) a b^{-1} \chi(\sigma) \\
& =a^{-1} b \psi(\sigma) \chi(\sigma) a b^{-1} \\
& =\left(a b^{-1}\right)^{-1} \psi^{\prime}(\sigma) a b^{-1}
\end{aligned}
$$

Therefore, $\left[\psi^{\prime \prime}\right]=\left[\psi^{\prime}\right]$ as desired.

Now suppose $x=1$, i.e. $x$ is represented by $\chi=1$. Hence $[\psi]^{x}=[\psi \chi]=[\psi]$. Thus $[\psi]^{1}=[\psi]$.

In a similar way, for each $\mathfrak{p} \in \mathbb{P}\left(K_{0}\right)$, the cohomology group $H^{1}\left(\operatorname{Gal}\left(K_{0, \mathfrak{p}}\right), A\right)$ acts on $\mathcal{H}_{\Gamma, \rho_{\mathfrak{p}}, \bar{\alpha}}\left(\operatorname{Gal}\left(K_{0, \mathfrak{p}}\right), \bar{G}\right)$. These actions satisfy

$$
[\psi]_{\mathfrak{p}}^{x}=\left[\psi_{\mathfrak{p}}\right]^{\operatorname{res}_{\mathfrak{p}}(x)}
$$

for $[\psi] \in \mathcal{H o m}_{\Gamma, \rho, \alpha}\left(\operatorname{Gal}\left(K_{0}\right), \bar{G}\right), x \in H^{1}\left(\operatorname{Gal}\left(K_{0}\right), A\right)$, and $\mathfrak{p} \in \mathbb{P}\left(K_{0}\right)$.

Recall that a principal homogeneous space $X$ over a group $H$ is a set $X$ on which $H$ acts freely and transitively (from the right). Thus, if $x \in X, \eta \in H$ and $x^{\eta}=x$, then $\eta=1$. Moreover, for all $y \in X$ there exists $\tau \in H$ such that $x^{\tau}=y$.

Lemma 4.1.2. The set $\mathcal{H} \operatorname{om}_{\Gamma, \rho, \bar{\alpha}}\left(\operatorname{Gal}\left(K_{0}\right), \bar{G}\right)\left(\operatorname{resp} . \mathcal{H} \mathrm{om}_{\Gamma, \rho_{\mathfrak{p}}, \bar{\alpha}}\left(\operatorname{Gal}\left(\hat{K}_{0, \mathfrak{p}}\right), \bar{G}\right)\right)$ is a principal homogeneous space over $H^{1}\left(\operatorname{Gal}\left(K_{0}\right), A\right)\left(\operatorname{resp} . H^{1}\left(\operatorname{Gal}\left(\hat{K}_{0, \mathfrak{p}}\right), A\right)\right)$, that is the action of $H^{1}\left(\operatorname{Gal}\left(K_{0}\right), A\right)\left(\operatorname{resp} \cdot H^{1}\left(\operatorname{Gal}\left(\hat{K}_{0, \mathfrak{p}}\right), A\right)\right)$ on $\mathcal{H o m}_{\Gamma, \rho, \bar{\alpha}}\left(\operatorname{Gal}\left(K_{0}\right), \bar{G}\right)$ (resp. $\left.\mathcal{H o m}_{\Gamma, \rho_{\mathfrak{p}}, \bar{\alpha}}\left(\operatorname{Gal}\left(\hat{K}_{0, \mathfrak{p}}\right), \bar{G}\right)\right)$ is transitive and free. 
Proof. Let $[\psi],[\varphi] \in \mathcal{H}_{\Gamma, \rho, \bar{\alpha}}\left(\operatorname{Gal}\left(K_{0}\right), \bar{G}\right)$ with representatives $\psi: \operatorname{Gal}\left(K_{0}\right) \longrightarrow$ $\bar{G}$ and $\varphi: \operatorname{Gal}\left(K_{0}\right) \longrightarrow \bar{G}$ respectively. Let $\chi: \operatorname{Gal}(K) \longrightarrow A$ be the map defined by $\chi(\sigma)=\varphi(\sigma)^{-1} \psi(\sigma)$ for each $\sigma \in \operatorname{Gal}\left(K_{0}\right)$. Since $\bar{\alpha}\left(\varphi(\sigma)^{-1} \psi(\sigma)\right)=$ $\bar{\alpha}\left(\varphi(\sigma)^{-1}\right) \bar{\alpha}(\psi(\sigma))=\rho\left(\sigma^{-1}\right) \rho(\sigma)=1$, we have $\varphi(\sigma)^{-1} \psi(\sigma) \in A$, so $\chi$ is well defined. Moreover, $\chi$ is a crossed homomorphism. Indeed, let $\sigma, \tau \in \operatorname{Gal}\left(K_{0}\right)$. Since $\bar{\alpha}(\varphi(\tau))=\rho(\tau)$, then

$$
\begin{aligned}
\chi(\sigma)^{\tau} \chi(\tau) & =\left(\varphi(\tau)^{-1} \varphi(\sigma)^{-1} \psi(\sigma) \varphi(\tau)\right)\left(\varphi(\tau)^{-1} \psi(\tau)\right) \\
& =\varphi(\tau)^{-1} \varphi(\sigma)^{-1} \psi(\sigma) \psi(\tau) \\
& =\varphi(\sigma \tau)^{-1} \psi(\sigma \tau) \\
& =\chi(\sigma \tau) .
\end{aligned}
$$

Let $x \in H^{1}\left(\operatorname{Gal}\left(K_{0}\right), A\right)$ be the cohomology class of $\chi$. Since $\psi(\sigma)=$ $\varphi(\sigma) \chi(\sigma)$ for each $\sigma \in \operatorname{Gal}\left(K_{0}\right)$, we have $[\psi]=[\varphi]^{x}$, so the action is transitive.

Now suppose that $[\varphi]^{x}=[\varphi]$ for some $x \in H^{1}\left(\operatorname{Gal}\left(K_{0}\right), A\right)$. Let $\chi$ be a representative of $x$. Then, there exists $a \in A$ such that $\varphi(\sigma) \chi(\sigma)=a^{-1} \varphi(\sigma) a$, for each $\sigma \in \operatorname{Gal}\left(K_{0}\right)$. Hence,

$$
\begin{aligned}
\chi(\sigma) & =\varphi(\sigma)^{-1} a^{-1} \varphi(\sigma) a \\
& =\left(a^{-1}\right)^{\sigma} a .
\end{aligned}
$$

Hence $\chi$ is a coboundary, i.e. $x=1$ in $H^{1}\left(\operatorname{Gal}\left(K_{0}\right), A\right)$. Thus, the action of $H^{1}\left(\operatorname{Gal}\left(K_{0}\right), A\right)$ on the set $\mathcal{H o m}_{\Gamma, \rho, \bar{\alpha}}\left(\operatorname{Gal}\left(K_{0}\right), \bar{G}\right)$ is free. Similar methods hold to prove that the action of $H^{1}\left(\operatorname{Gal}\left(\hat{K}_{0, \mathfrak{p}}\right), A\right)$ on the set $\mathcal{H} \operatorname{om}_{\Gamma, \rho_{\mathfrak{p}}, \bar{\alpha}}\left(\operatorname{Gal}\left(\hat{K}_{0, \mathfrak{p}}\right), \bar{G}\right)$ is transitive and free.

Lemma 4.1.3. Let $\mathfrak{p} \in \mathbb{P}\left(K_{0}\right)$. Let $[\psi] \in \mathcal{H}^{\circ} \mathrm{om}_{\Gamma, \rho, \bar{\alpha}}\left(\operatorname{Gal}\left(K_{0}\right), \bar{G}\right)$. Suppose $[\psi]$ is unramfied at $\mathfrak{p}$. Let $x \in H^{1}\left(\operatorname{Gal}\left(K_{0}\right), A\right)$ such that $x_{\mathfrak{p}}=\operatorname{res}_{\mathfrak{p}}(x) \in$ $H^{1}\left(\operatorname{Gal}\left(\hat{K}_{0, \mathfrak{p}}\right), A\right)$ is unramified. Then $\left[\psi^{\prime}\right]=[\psi]^{x}$ is unramified at $\mathfrak{p}$.

Proof. Let $\psi$ and $\chi$ be representatives of $[\psi]$ and $x$ respectively. Hence $\psi \cdot \chi$ is a representative of $\left[\psi^{\prime}\right]$. Let $\chi_{\mathfrak{p}}: \operatorname{Gal}\left(\hat{K}_{0, \mathfrak{p}}\right) \longrightarrow A$ be a representative 
of $x_{\mathfrak{p}}$ in $\mathcal{Z}^{1}\left(\operatorname{Gal}\left(\hat{K}_{0, \mathfrak{p}}\right), A\right)$. Since $x_{\mathfrak{p}}$ is unramified, there exists $a_{\mathfrak{p}} \in A$ such that for each $\sigma \in \hat{I}_{\mathfrak{p}}, \chi_{\mathfrak{p}}(\sigma)=a_{\mathfrak{p}}^{\sigma} a_{\mathfrak{p}}^{-1}$ (Definition 3.1.2(a)). Hence for each $\sigma \in \hat{I}_{\mathfrak{p}}$

$$
\begin{aligned}
\psi^{\prime}(\sigma) & =\psi(\sigma) \chi_{\mathfrak{p}}(\sigma) \\
& =\psi(\sigma) a_{\mathfrak{p}}^{\sigma} a_{\mathfrak{p}}^{-1} \\
& =a_{\mathfrak{p}}^{\sigma} a_{\mathfrak{p}}^{-1},
\end{aligned}
$$

because $\psi(\sigma)=1$. Now $a_{\mathfrak{p}}^{\sigma}=a_{\mathfrak{p}}^{\rho(\sigma)}$. Since $\bar{\alpha}(\psi(\sigma))=\rho(\sigma)$, we have $a_{\mathfrak{p}}^{\sigma}=$ $\psi(\sigma)^{-1} a_{\mathfrak{p}} \psi(\sigma)=a_{\mathfrak{p}}$. It follows that $\psi^{\prime}(\sigma)=a_{\mathfrak{p}} a_{\mathfrak{p}}^{-1}=1$, for all $\sigma \in \hat{I}_{\mathfrak{p}}$. Thus $\left[\psi^{\prime}\right]$ is unramified at $\mathfrak{p}$.

\subsection{Embedding Problem whose Kernel is a Simple $\operatorname{Gal}\left(K_{0}\right)$-module}

In this section we solve the first layer of our embedding problem. We construct an epimorphism $\bar{\psi}: \operatorname{Gal}\left(K_{0}\right) \longrightarrow \bar{G}$ in such a way that the induced second layer of the embedding problem is locally solvable, if $\bar{N}$ is the solution field (fixed field of $\operatorname{Ker}(\bar{\psi})$ in $\tilde{K}_{0}$ ) then $|\mu(\bar{N})|$ and the order of the kernel of the next layer are coprime, and we have a bound on $|\operatorname{Ram}(\bar{\psi})|$.

Let $K / K_{0}$ be a finite Galois extension of number fields with $\operatorname{Gal}\left(K / K_{0}\right)=\Gamma$, let $\bar{\alpha}: \bar{G} \longrightarrow \Gamma$ be an epimorphism with kernel $A=C_{l}^{r}$ which is a simple $\Gamma$-module. Suppose that $\zeta_{l} \notin K$. We use the action of $\operatorname{Gal}\left(K_{0}\right)$ on $A$ defined in (4.1.3), to make $A$ a simple $\operatorname{Gal}\left(K_{0}\right)$-module and $\operatorname{Gal}(K)$ acts trivially on A.

We start with a Lemma that will assure the surjectivity of the solution $\bar{\psi}$ and handles the growth of the number of roots of unity in the solution field.

Lemma 4.2.1. Consider the embedding problem in 4.1.1). Let $n$ be a positive integer. Let $m$ be the minimal number of generators of $\operatorname{Gal}\left(K\left(\zeta_{n}\right) / K\right)$. 
Suppose that $\operatorname{gcd}(n,|\mu(K)|)=1$. Let $T$ be a finite set of primes of $K_{0}$. There exist distinct primes $\mathfrak{p}_{1}, \ldots, \mathfrak{p}_{m}, \mathfrak{q} \in \mathbb{P}\left(K_{0}\right) \backslash T$ which totally split in $K$ and for each $\mathfrak{p} \in\left\{\mathfrak{p}_{1}, \ldots, \mathfrak{p}_{m}, \mathfrak{q}\right\}$ there exists an element $\left[\varphi_{\mathfrak{p}}\right] \in \mathcal{H} \operatorname{om}_{\Gamma, \rho_{\mathfrak{p}}, \bar{\alpha}}\left(\operatorname{Gal}\left(\hat{K}_{0, \mathfrak{p}}\right), \bar{G}\right)$ such that if an element $[\bar{\psi}] \in \mathcal{H}_{\Gamma, \rho, \bar{\alpha}}\left(\operatorname{Gal}\left(K_{0}\right), \bar{G}\right)$, satisfies $\left[\bar{\psi}_{\mathfrak{p}}\right]=\left[\varphi_{\mathfrak{p}}\right]$ in $\mathcal{H} \mathrm{om}_{\Gamma, \rho_{\mathfrak{p}}, \bar{\alpha}}\left(\operatorname{Gal}\left(K_{0, \mathfrak{p}}\right), \bar{G}\right)$ then

(a) $[\bar{\psi}]$ is unramified at $\mathfrak{p}_{1}, \ldots, \mathfrak{p}_{m}, \mathfrak{q}$,

(b) if $\bar{N}$ is the fixed field of $\operatorname{Ker}(\bar{\psi})$ in $\tilde{K}_{0}$ then $\operatorname{gcd}(n,|\mu(\bar{N})|)=1$,

(c) $[\bar{\psi}]$ is surjective.

Proof. We break up the proof into several parts.

Part A: The choice of $\mathfrak{p}_{1}, \ldots, \mathfrak{p}_{m}$. Let $\operatorname{Gal}\left(K\left(\zeta_{n}\right) / K\right)=\left\langle\sigma_{1}, \ldots, \sigma_{m}\right\rangle$. Let $T_{K\left(\zeta_{n}\right)}$ be the finite set of primes of $K\left(\zeta_{n}\right)$ that divide the primes in $T$. We apply the Chebotarev density theorem and choose, by induction on $m$, primes $\mathfrak{Q}_{1}, \ldots, \mathfrak{Q}_{m} \in \mathbb{P}\left(K\left(\zeta_{n}\right)\right) \backslash T_{K\left(\zeta_{n}\right)}$ in such a way that $\left[\frac{K\left(\zeta_{n}\right) / K}{\mathfrak{Q}_{i}}\right]=\sigma_{i}$ for $i=1, \ldots, m$ and the primes $\mathfrak{p}_{1}=\left.\mathfrak{Q}_{1}\right|_{K_{0}}, \ldots, \mathfrak{p}_{m}=\left.\mathfrak{Q}_{m}\right|_{K_{0}} \in \mathbb{P}\left(K_{0}\right) \backslash T$ are distinct. For each $i=1, \ldots, m$ set $\mathfrak{P}_{i}=\left.\mathfrak{Q}_{i}\right|_{K}$. Then $\left[\frac{K / K_{0}}{\mathfrak{P}_{i}}\right]=\left.\sigma_{i}\right|_{K}=1$, so $\mathfrak{p}_{i}$ totally splits in $K$ (last paragraph of Section 1.2). Thus, $K \subset \hat{K}_{0, \mathfrak{p}_{i}}$. Since $\operatorname{Gal}(K)=\operatorname{Ker}(\rho)$, we have that $\rho_{\mathfrak{p}_{i}}: \operatorname{Gal}\left(\hat{K}_{0, \mathfrak{p}_{i}}\right) \longrightarrow \Gamma$ is trivial. Hence, the class $\left[\varphi_{\mathfrak{p}_{i}}\right]$ of the trivial homomorphism $\varphi_{\mathfrak{p}_{i}}: \operatorname{Gal}\left(\hat{K}_{0, \mathfrak{p}_{i}}\right) \longrightarrow \bar{G}$ is an element of $\mathcal{H} \mathrm{om}_{\Gamma, \rho_{\mathfrak{p}_{i}}, \bar{\alpha}}\left(\operatorname{Gal}\left(\hat{K}_{0, \mathfrak{p}_{i}}\right), \bar{G}\right)$ for each $i=1, \ldots, m$.

Part B: The choice of the prime $\mathfrak{q}$. By the Chebotarev density theorem, there exists $\mathfrak{q} \in \mathbb{P}\left(K_{0}\right) \backslash\left(T \bullet\left\{\mathfrak{p}_{1}, \ldots, \mathfrak{p}_{m}\right\}\right)$ which totally splits in $K$. Let $\hat{K}_{0, \mathfrak{q} \text {,ur }}$ be the maximal unramified extension of $\hat{K}_{0, \mathfrak{q}}$. Then $\operatorname{Gal}\left(\hat{K}_{0, \mathfrak{q}, \mathrm{ur}} / \hat{K}_{0, \mathfrak{q}}\right) \cong \hat{\mathbb{Z}}$. Let $\sigma$ be the generator of $\operatorname{Gal}\left(\hat{K}_{0, \mathfrak{q}, \text { ur }} / \hat{K}_{0, \mathfrak{q}}\right)$ that corresponds under this isomorphism to 1 of $\hat{\mathbb{Z}}$. Let $a \in A$ with $a \neq 1$. Then there exists unique homomorphism $\bar{\varphi}_{\mathfrak{q}}: \operatorname{Gal}\left(\hat{K}_{0, \mathfrak{q}, \mathrm{ur}} / \hat{K}_{0, \mathfrak{q}}\right) \longrightarrow \bar{G}$ that maps $\sigma$ to $a$. Consider the homomorphism $\varphi_{\mathfrak{q}}: \operatorname{Gal}\left(\hat{K}_{0, \mathfrak{q}}\right) \stackrel{\text { res }}{\longrightarrow} \operatorname{Gal}\left(\hat{K}_{0, \mathfrak{q}, \text { ur }} / \hat{K}_{0, \mathfrak{q}}\right) \stackrel{\bar{\varphi}_{\mathfrak{q}}}{\longrightarrow} \bar{G}$. Since $\hat{I}_{\mathfrak{q}}=\operatorname{Ker}\left(\operatorname{Gal}\left(\hat{K}_{0, \mathfrak{q}}\right) \longrightarrow \operatorname{Gal}\left(\hat{K}_{0, \mathfrak{q}, \text { ur }} / \hat{K}_{0, \mathfrak{q}}\right)\right)$, we have $\varphi_{\mathfrak{q}}\left(\hat{I}_{\mathfrak{q}}\right)=1$, that is $\varphi_{\mathfrak{q}}$ is 
unramified. On the other hand, since $\mathfrak{q}$ totally splits in $K$, we have, as in Part A, that $\rho_{\mathfrak{q}}: \operatorname{Gal}\left(\hat{K}_{0, \mathfrak{q}}\right) \longrightarrow \Gamma$ is the trivial homomorphism. If $\tau \in \operatorname{Gal}\left(\hat{K}_{0, \mathfrak{q}}\right)$, there exists $s \in \mathbb{Z}$ such that $\varphi_{\mathfrak{q}}(\tau)=a^{s}$, hence $\bar{\alpha} \circ \varphi_{\mathfrak{q}}(\tau)=\bar{\alpha}\left(a^{s}\right)=1=\rho_{\mathfrak{q}}(\tau)$. It follows that $\left[\varphi_{\mathfrak{q}}\right] \in \mathcal{H} \operatorname{om}_{\Gamma, \rho_{\mathfrak{q}}, \bar{\alpha}}\left(\operatorname{Gal}\left(\hat{K}_{0, \mathfrak{q}}\right), \bar{G}\right)$.

Part C: The conditions of the Lemma. Now let $[\bar{\psi}] \in \mathcal{H}_{\Gamma, \rho, \bar{\alpha}}\left(\operatorname{Gal}\left(K_{0}\right), \bar{G}\right)$ such that $\left[\bar{\psi}_{\mathfrak{p}}\right]=\left[\varphi_{\mathfrak{p}}\right]$ for $\mathfrak{p} \in\left\{\mathfrak{p}_{1}, \ldots, \mathfrak{p}_{m}, \mathfrak{q}\right\}$. Let $\bar{\psi}$ be a representative of $[\bar{\psi}]$, in particular $\bar{\alpha} \circ \bar{\psi}=\rho$.

Proof of (a). For each $\mathfrak{p} \in\left\{\mathfrak{p}_{1}, \ldots, \mathfrak{p}_{m}\right\},\left[\varphi_{\mathfrak{p}}\right]$ is the class of the trivial homomorphism, so it is unramified. Hence $\bar{\psi}$ is unramified at $\mathfrak{p}_{1}, \ldots, \mathfrak{p}_{m}$. Furthermore, by the definition of $\left[\varphi_{\mathfrak{q}}\right]$ in Part B to be unramified and since $\left[\bar{\psi}_{\mathfrak{q}}\right]=\left[\varphi_{\mathfrak{q}}\right]\left(\operatorname{Ker}\left(\bar{\psi}_{\mathfrak{q}}\right)=\operatorname{Ker}\left(\psi_{\mathfrak{q}}\right)\right)$, then $\bar{\psi}$ is unramified at $\mathfrak{q}$, as desired.

Proof of (b). Since $\bar{\alpha} \circ \bar{\psi}=\rho$, we have $\operatorname{Ker}(\bar{\psi}) \leq \operatorname{Ker}(\rho)=\operatorname{Gal}(K)$. Hence, the fixed field $\bar{N}$ of $\operatorname{Ker}(\bar{\psi})$ contains $K$. Therefore, $K \subseteq \bar{N} \cap K\left(\zeta_{n}\right)$. In addition, $\left[\bar{\psi}_{\mathfrak{p}_{i}}\right]=\left[\varphi_{\mathfrak{p}_{i}}\right]$ and $\varphi_{\mathfrak{p}_{i}}$ is trivial, so $\bar{\psi}_{\mathfrak{p}_{i}}$ is trivial, that is $\bar{\psi}\left(\operatorname{Gal}\left(\hat{K}_{0, \mathfrak{p}_{i}}\right)\right)=1$, so $\bar{N} \subset \hat{K}_{0, \mathfrak{p}_{i}}$ for each $i=1, \ldots, m$. Hence $\mathfrak{p}_{i}$ totally splits in $\bar{N}$, so in $\bar{N} \cap K\left(\zeta_{n}\right)$. Therefore, the automorphisms $\bar{\sigma}_{i}=\left[\frac{\bar{N} \cap K\left(\zeta_{n}\right) / K}{\mathfrak{P}_{i}}\right], i=1, \ldots, m$, which generate the Galois group $\operatorname{Gal}\left(\bar{N} \cap K\left(\zeta_{n}\right) / K\right)$, are all identity. It follows that $\bar{N} \cap K\left(\zeta_{n}\right) \subseteq K$, hence $\bar{N} \cap K\left(\zeta_{n}\right)=K$. Let now $d=\operatorname{gcd}(n,|\mu(\bar{N})|)$ and let $\zeta_{d}$ be a primitive $d$-th root of unity. Then $\zeta_{d} \in \bar{N} \cap K\left(\zeta_{n}\right)=K$. Thus $d \mid \operatorname{gcd}(n,|\mu(K)|)$, so $d=1$.

Proof of $(c)$. Since $\left[\bar{\psi}_{\mathfrak{q}}\right]=\left[\varphi_{\mathfrak{q}}\right]$, there exists $b \in A$, with $\bar{\psi}_{\mathfrak{q}}(\tau)=b^{-1} \varphi_{\mathfrak{q}}(\tau) b$, for all $\tau \in \operatorname{Gal}\left(\hat{K}_{0, \mathfrak{q}}\right)$. Since $a \in \varphi_{\mathfrak{q}}\left(\operatorname{Gal}\left(\hat{K}_{0, \mathfrak{q}}\right)\right)$ (chosen in Part B), and $A$ is abelian, $a \in \bar{\psi}\left(\operatorname{Gal}\left(\hat{K}_{0, \mathfrak{q}}\right)\right) \subseteq \bar{\psi}\left(\operatorname{Gal}\left(K_{0}\right)\right)$. Therefore $A \cap \bar{\psi}\left(\operatorname{Gal}\left(K_{0}\right)\right)$ is a non-trivial $\Gamma$-submodule of $A$. Since $A$ is simple, $A \subseteq \bar{\psi}\left(\operatorname{Gal}\left(K_{0}\right)\right)$. Since $\rho: \operatorname{Gal}\left(K_{0}\right) \longrightarrow \Gamma$ is surjective, $\bar{\psi}\left(\operatorname{Gal}\left(K_{0}\right)\right)=\bar{G}$.

(4.2.1) The arithmetical function $\Omega: \mathbb{N} \longrightarrow \mathbb{N}$ is defined for $n=\prod_{i=1}^{m} l_{i}^{r_{i}}$, 
where $l_{1}, \ldots, l_{m}$ are distinct prime divisors, by $\Omega(n)=\sum_{i=1}^{m} r_{i}$. Therefore, $\Omega(n m)=\Omega(n)+\Omega(m)$ (See [HaW62], p. 354, Sec. 22.10).

Now we prove the main result of this section. For the next layer, we consider an epimorphism of $\gamma: G \rightarrow \bar{G}$ of finite groups with solvable kernel.

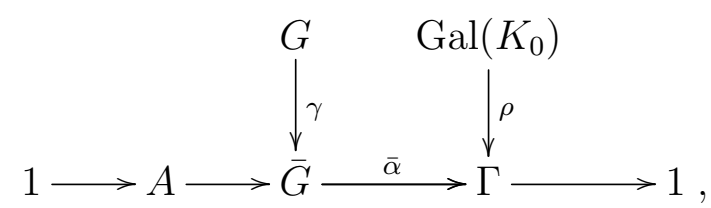

Proposition 4.2.2. Consider the embedding problem in 4.2.2 with the properties as in (4.1.2), and an epimorphism $\gamma: G \longrightarrow \bar{G}$ with solvable kernel from a finite group $G$. Let $n$ be a positive integer multiple of $|\operatorname{Ker}(\gamma)| l$. Let $T$ be a finite set of primes of $K_{0}$ such that $\operatorname{Ram}\left(K / K_{0}\right) \subseteq T$. Suppose $\operatorname{gcd}(n,|\mu(K)|)=1$, and $\prod_{\mathfrak{p}} \mathcal{H} \operatorname{om}_{\Gamma, \rho_{\mathfrak{p}}, \bar{\alpha}}\left(\operatorname{Gal}\left(\hat{K}_{0, \mathfrak{p}}\right), \bar{G}\right) \neq \emptyset$. For each $\mathfrak{p} \in T$, let $\left[\varphi_{\mathfrak{p}}\right] \in \mathcal{H} \operatorname{om}_{\Gamma, \rho_{\mathfrak{p}}, \bar{\alpha}}\left(\operatorname{Gal}\left(\hat{K}_{0, \mathfrak{p}}\right), \bar{G}\right)$. There exists a set $R \subset \mathbb{P}\left(K_{0}\right) \backslash T$ with $|R|=\Omega(|A|)$ and there exists an element $[\bar{\psi}] \in \mathcal{H}_{\Gamma, \rho, \bar{\alpha}}\left(\operatorname{Gal}\left(K_{0}\right), \bar{G}\right)_{\text {sur }}$ such that

(a) $\left[\bar{\psi}_{\mathfrak{p}}\right]=\left[\varphi_{\mathfrak{p}}\right]$ in $\mathcal{H}_{\Gamma, \rho_{\mathfrak{p}}, \bar{\alpha}}\left(\operatorname{Gal}\left(\hat{K}_{0, \mathfrak{p}}\right), \bar{G}\right)$ for each $\mathfrak{p} \in T$,

(b) $[\bar{\psi}]$ is unramified at each $\mathfrak{p} \in \mathbb{P}\left(K_{0}\right) \backslash(T \bullet R)$, that is if $\bar{N}$ is the solution field (fixed field of $\operatorname{Ker}(\bar{\psi})$ ) then $\operatorname{Ram}\left(\bar{N} / K_{0}\right) \subset T \bullet R$,

(c) $\left[\bar{\psi}_{\mathfrak{p}}\right]$ can be lifted to an element $\left[\psi_{\mathfrak{p}}\right] \in \mathcal{H}^{\circ} \mathrm{m}_{\bar{G}, \bar{\psi}_{\mathfrak{p}, \gamma}}\left(\operatorname{Gal}\left(\hat{K}_{0, \mathfrak{p}}\right), G\right)$ for each $\mathfrak{p} \in \mathbb{P}\left(K_{0}\right) \backslash T$, and

(d) $\operatorname{gcd}(n,|\mu(\bar{N})|)=1$.

Proof. Assume without loss that the basic set $S_{0} \subseteq T_{K}$ where $T_{K}$ is the set of primes of $K$ that lie over the primes in $T$. We break up the proof into three parts.

Part A: The surjectivity and the number of roots of unity. Let $m$ be the minimal number of generators of $\operatorname{Gal}\left(K\left(\zeta_{n}\right) / K\right)$. We choose $\mathfrak{p}_{1}, \ldots, \mathfrak{p}_{m}, \mathfrak{q} \in$ 
$\mathbb{P}\left(K_{0}\right) \backslash T$ and elements $\left[\varphi_{\mathfrak{p}}\right]$ in the set $\mathcal{H}_{\Gamma, \rho_{\mathfrak{p}}, \bar{\alpha}}\left(\operatorname{Gal}\left(\hat{K}_{0, \mathfrak{p}}\right), \bar{G}\right)$ for each $\mathfrak{p} \in\left\{\mathfrak{p}_{1}, \ldots, \mathfrak{p}_{m}, \mathfrak{q}\right\}$ that satisfy the conditions of Lemma 4.2.1. If $[\bar{\psi}] \in$ $\mathcal{H o m}_{\Gamma, \rho, \bar{\alpha}}\left(\operatorname{Gal}\left(K_{0}\right), \bar{G}\right)$ satisfies $\left[\bar{\psi}_{\mathfrak{p}}\right]=\left[\varphi_{\mathfrak{p}}\right]$ for each $\mathfrak{p} \in\left\{\mathfrak{p}_{1}, \ldots, \mathfrak{p}_{m}, \mathfrak{q}\right\}$, then by Lemma 4.2.1.

(4.2.3) $[\bar{\psi}]$ is unramfied at $\mathfrak{q}, \mathfrak{p}_{1}, \ldots, \mathfrak{p}_{m}$,

(4.2.4) the fixed field $\bar{N}$ of $\operatorname{Ker}(\bar{\psi})$ satisfies $\operatorname{gcd}(n,|\mu(\bar{N})|)=1$, and $(4.2 .5)[\bar{\psi}]$ is surjective.

Since $\prod_{\mathfrak{p}} \mathcal{H} \mathrm{om}_{\Gamma, \rho_{\mathfrak{p}}, \bar{\alpha}}\left(\operatorname{Gal}\left(\hat{K}_{0, \mathfrak{p}}\right), \bar{G}\right) \neq \emptyset$, by Lemma 1.2 .8 , there exists an element $\left[\psi_{0}\right] \in \mathcal{H}_{\Gamma, \rho, \bar{\alpha}}\left(\operatorname{Gal}\left(K_{0}\right), \bar{G}\right)$. Our aim is to change $\left[\psi_{0}\right]$ by the action of an element of $H^{1}\left(\operatorname{Gal}\left(K_{0}\right), A\right)$ to an element $[\bar{\psi}]$ which satisfies the conclusion of the Proposition.

Let $N_{0}$ be the fixed field of the kernel of $\psi_{0}: \operatorname{Gal}\left(K_{0}\right) \longrightarrow \bar{G}$. Then, $N_{0}$ is a finite Galois extension of $K_{0}$ that contains $K$ such that $N_{0} / K$ is an abelian $l$-extension $\left(\operatorname{Gal}\left(N_{0} / K\right) \leq A\right)$.

Part B: The sets $T^{*}$ and $T^{* *}$. Let $T^{*}=T \bullet\left\{\mathfrak{q}, \mathfrak{p}_{1}, \ldots, \mathfrak{p}_{m}\right\}$. Let $\mathfrak{u}_{1}, \ldots, \mathfrak{u}_{v} \in$ $\mathbb{P}\left(K_{0}\right) \backslash T^{*}$ be the primes where $\psi_{0}$ ramifies. Then each $\mathfrak{u}_{i}$ is unramified in $K$. It follows from Lemma 1.2.6 that the unramified homomorphism $\rho_{\mathfrak{u}_{i}}: \operatorname{Gal}\left(\hat{K}_{0, \mathfrak{u}_{i}}\right) \longrightarrow \Gamma$ can be lifted to an element $\left[\varphi_{\mathfrak{u}_{i}}\right] \in \mathcal{H} \mathrm{om}_{\Gamma, \rho_{\mathfrak{u}_{i}}, \bar{\alpha}}\left(\operatorname{Gal}\left(\hat{K}_{0, \mathfrak{u}_{i}}\right), \bar{G}\right)$ which is unramified for each $i=1, \ldots, v$. Hence, if $[\bar{\psi}] \in \mathcal{H}_{\Gamma, \rho, \bar{\alpha}}\left(\operatorname{Gal}\left(K_{0}\right), \bar{G}\right)$ satisfies $\left[\bar{\psi}_{\mathfrak{u}_{i}}\right]=\left[\varphi_{\mathfrak{u}_{i}}\right]$ for each $i=1, \ldots, v$, then:

(4.2.6) $[\bar{\psi}]$ is unramified at $\mathfrak{u}_{1}, \ldots, \mathfrak{u}_{v}$.

For each $\mathfrak{p} \in \mathbb{P}\left(K_{0}\right)$, let $\psi_{0 \mathfrak{p}}: \operatorname{Gal}\left(\hat{K}_{0, \mathfrak{p}}\right) \longrightarrow G$ be the restriction of $\psi_{0}$ to $\operatorname{Gal}\left(\hat{K}_{0, \mathfrak{p}}\right)$. Let $T^{* *}=T^{*} \bullet\left\{\mathfrak{u}_{1}, \ldots, \mathfrak{u}_{v}\right\}$. Then

(4.2.7) $\psi_{0}$ is unramified at each $\mathfrak{p} \in \mathbb{P}\left(K_{0}\right) \backslash T^{* *}$.

Consider the system $\left(\left[\varphi_{\mathfrak{p}}\right] \in \mathcal{H} \mathrm{om}_{\Gamma, \rho_{\mathfrak{p}}, \alpha}\left(\operatorname{Gal}\left(\hat{K}_{0, \mathfrak{p}}\right), G\right)\right)_{\mathfrak{p} \in T^{* *}}$. For each $\mathfrak{p} \in T^{* *}$, by Lemma 4.1.2, there exists a unique element $y_{\mathfrak{p}} \in H^{1}\left(\operatorname{Gal}\left(\hat{K}_{0, \mathfrak{p}}\right), A\right)$ that 
satisfies:

$$
\left[\psi_{0 \mathfrak{p}}\right]^{y_{\mathfrak{p}}}=\left[\varphi_{\mathfrak{p}}\right]
$$

The module $A$ is a simple $\operatorname{Gal}\left(K_{0}\right)$-module and $\operatorname{Gal}(K)$ acts trivially on $A$. Furthermore, the set $S_{0}$ is contained in the set $T_{K}^{* *}$ of all primes of $K$ that lie over $T^{* *}$. Since $\operatorname{gcd}(n,|\mu(K)|)=1$, we have $\zeta_{l} \notin K$. Moreover, since $N_{0} / K$ is an abelian $l$-extension, Proposition 3.3 .5 gives an element $x \in H^{1}\left(\operatorname{Gal}\left(K_{0}\right), A\right)$ and primes $\mathfrak{q}_{1}, \ldots, \mathfrak{q}_{r} \in \mathbb{P}\left(K_{0}\right) \backslash T^{* *}$ such that:

$$
x_{\mathfrak{p}}=y_{\mathfrak{p}} \text { for } \mathfrak{p} \in T^{* *}
$$

(4.2.10) $x_{\mathfrak{p}}$ is unramified for each $\mathfrak{p} \in \mathbb{P}\left(K_{0}\right) \backslash\left(T^{* *} \sqcup\left\{\mathfrak{q}_{1}, \ldots, \mathfrak{q}_{r}\right\}\right)$.

(4.2.11) for $i=1, \ldots, r, \mathfrak{q}_{i}$ totally splits in $N_{0}\left(\zeta_{n}\right)$, and $x_{\mathfrak{q}_{i}}: \operatorname{Gal}\left(\hat{K}_{0, \mathfrak{q}_{i}}\right) \longrightarrow C_{l}$ is a cyclic homomorphism,

(4.2.12) for each $i=1, \ldots, r, x_{\mathfrak{q}_{i}}$ can be lifted to a $\bar{G}$-homomorphism $x_{\mathfrak{q}_{i}}^{\prime}: \operatorname{Gal}\left(\hat{K}_{0, \mathfrak{q}_{i}}\right) \rightarrow G$ (i.e. $\left.\gamma \circ x_{\mathfrak{q}_{i}}^{\prime}=x_{\mathfrak{q}_{i}}\right)$.

Part C: The solution $\bar{\psi}$. We prove that the element $[\bar{\psi}]=\left[\psi_{0}\right]^{x}$ of $\mathcal{H o m}_{\Gamma, \rho, \bar{\alpha}}\left(\operatorname{Gal}\left(K_{0}\right), \bar{G}\right)$ satisfies the conclusion of the Proposition.

For each $\mathfrak{p} \in T^{* *}$, by (4.2.9) and (4.2.8):

$$
\left[\bar{\psi}_{\mathfrak{p}}\right]=\left[\psi_{0 \mathfrak{p}}\right]^{x_{\mathfrak{p}}}=\left[\psi_{0 \mathfrak{p}}\right]^{y_{\mathfrak{p}}}=\left[\varphi_{\mathfrak{p}}\right] \text { in } \mathcal{H} \mathrm{om}_{\Gamma, \rho_{\mathfrak{p}}, \bar{\alpha}}\left(\operatorname{Gal}\left(\hat{K}_{0, \mathfrak{p}}\right), \bar{G}\right)
$$

Proof of (a): Since $T \subseteq T^{* *}$, by 4.2.13, Condition (a) holds.

Proof of $(b)$ : Denote $R=\left\{\mathfrak{q}_{1}, \ldots, \mathfrak{q}_{r}\right\}$. Let $\mathfrak{p} \in \mathbb{P}\left(K_{0}\right) \backslash(T \bullet R)$. If $\mathfrak{p} \in T^{* *}$ (that is $\mathfrak{p} \in\left\{\mathfrak{q}, \mathfrak{p}_{1}, \ldots, \mathfrak{p}_{m}, \mathfrak{u}_{1}, \ldots, \mathfrak{u}_{v}\right\}$ ), then, by (4.2.3) and $(4.2 .6)$, $[\bar{\psi}]$ is unramified at $\mathfrak{p}$. If $\mathfrak{p} \in \mathbb{P}\left(K_{0}\right) \backslash\left(T^{* *} \bullet\left\{\mathfrak{q}_{1}, \ldots, \mathfrak{q}_{r}\right\}\right)$, then by (4.2.7)] and $(4.2 .10),\left[\psi_{0 \mathfrak{p}}\right]$ and $x_{\mathfrak{p}}$ are unramified. Hence, by Lemma 4.1.3, $\left[\bar{\psi}_{\mathfrak{p}}\right]=\left[\psi_{0 \mathfrak{p}}\right]^{x_{\mathfrak{p}}}$ is unramified. So Condition (b) holds. Note that $|R|=\Omega(|A|)$. 
Proof of $(c)$ : Let $\mathfrak{p} \in \mathbb{P}\left(K_{0}\right) \backslash T$. If $\mathfrak{p} \notin R$, then by (b), [ $\left.\bar{\psi}_{\mathfrak{p}}\right]$ is unramified. It follows from Lemma 1.2 .6 that $\bar{\psi}_{\mathfrak{p}}$ can be lifted to an unramified element of $\mathcal{H} \mathrm{om}_{\bar{G}, \bar{\psi}_{\mathfrak{p}}, \gamma}\left(\operatorname{Gal}\left(\hat{K}_{0, \mathfrak{p}}\right), G\right)$. If $\mathfrak{p} \in R$, then, by (4.2.11), $\mathfrak{p}$ totally splits in $N_{0}\left(\zeta_{n}\right)$, in particular in $N_{0}$. Hence $\psi_{0 \mathfrak{p}}: \operatorname{Gal}\left(\hat{K}_{0, \mathfrak{p}}\right) \longrightarrow \bar{G}$ is the trivial homomorphism. Furthermore, by (4.2.11), the homomorphism $x_{\mathfrak{p}}: \operatorname{Gal}\left(\hat{K}_{0, \mathfrak{p}}\right) \longrightarrow C_{l}$ satisfies

$$
\left[\bar{\psi}_{\mathfrak{p}}\right]=\left[\psi_{0 \mathfrak{p}}\right]^{x_{\mathfrak{p}}}=\left[\psi_{0 \mathfrak{p}} \cdot x_{\mathfrak{p}}\right]=\left[x_{\mathfrak{p}}\right]
$$

because $x_{\mathfrak{p}}$ represents its own class. By (4.2.12), $x_{\mathfrak{p}}$ can be lifted to a $\bar{G}$-homomorphism $x_{\mathfrak{p}}^{\prime}$. Hence, also $\bar{\psi}_{\mathfrak{p}}$ has the same property. Therefore, $\mathcal{H}_{\mathrm{om}_{\bar{G}}, \bar{\psi}_{\mathfrak{p}, \gamma}}\left(\operatorname{Gal}\left(\hat{K}_{0, \mathfrak{p}}\right), G\right) \neq \emptyset$.

Proof of (d): From (4.2.4), the fixed field $\bar{N}$ of $\bar{\psi}$ satisfies $\operatorname{gcd}(n,|\mu(\bar{N})|)=1$. And by (4.2.5), $\bar{\psi}$ is surjective, and this completes the proof.

The epimorphism $\bar{\psi}: \operatorname{Gal}\left(K_{0}\right) \rightarrow \bar{G}$ defined in Proposition 4.2 .2 gives rise to an embedding problem

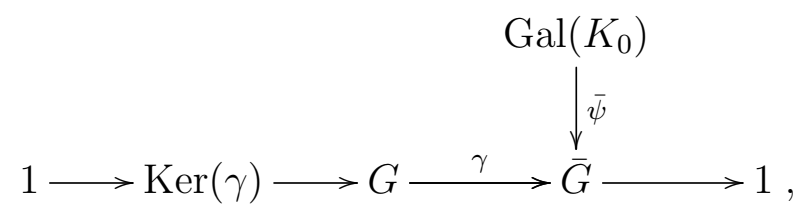

with solvable kernel which will be the next layer. The condition $\prod_{\mathfrak{p}} \mathcal{H} \operatorname{om}_{\bar{G}, \bar{\psi}_{\mathfrak{p}}, \gamma}\left(\operatorname{Gal}\left(\hat{K}_{0, \mathfrak{p}}\right), G\right) \neq \emptyset$ is necessary in order that the global embedding problem has a solution. By (c) of that Proposition, for each $\mathfrak{p} \in$ $\mathbb{P}\left(K_{0}\right) \backslash T, \mathcal{H}_{\bar{G}, \bar{\psi}_{\mathfrak{p}, \gamma}}\left(\operatorname{Gal}\left(\hat{K}_{0, \mathfrak{p}}\right), G\right) \neq \emptyset$. For $\mathfrak{p} \in T$, in the next section, the properties of the epimorphism $\gamma$ and the equality $\left[\bar{\psi}_{\mathfrak{p}}\right]=\left[\varphi_{\mathfrak{p}}\right]$ as in (a) of the Proposition will allow us to show the solvability of the corresponding local embedding problem. 


\subsection{Embedding Problem with Solvable Kernel}

We are now ready to prove the main result of this work. Our approach is similar to that of [Neu79]. However we give an explicit bound on the ramification of our solution based on the bound given in the first layer (Proposition 4.2 .2 .

Construction 4.3.1. Consider the following finite embedding problem, where $H=\operatorname{Ker}(\alpha)$ is solvable,

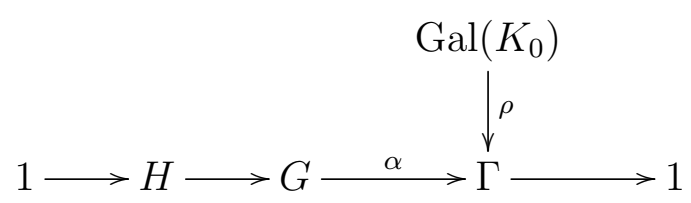

It suffices to solve the problem in the case where $H$ is non-trivial. To simplify the notation, for each $a \in H$ and each $\Gamma$-homomorphism $\varphi: \operatorname{Gal}\left(K_{0}\right) \longrightarrow G$, let $\varphi^{a}: \operatorname{Gal}\left(K_{0}\right) \longrightarrow G$ be the homomorphism defined for each $\sigma \in \operatorname{Gal}\left(K_{0}\right)$ by $\varphi^{a}(\sigma)=a^{-1} \varphi(\sigma) a$ (i.e. $\varphi^{a}$ and $\varphi$ are $\operatorname{Ker}(\alpha)$-conjugate).

Since $H$ is solvable, it has a normal subgroup $H_{1}$ such that $H / H_{1}$ is a nontrivial abelian group. Note that for each $g \in G, H_{1}^{g}=g^{-1} H_{1} g$ is a normal subgroup of $H$ with $H / H_{1}^{g}$ is abelian. The subgroup $\bigcap_{g \in G} H_{1}^{g}$ is the kernel of the homomorphism $\theta: H \longrightarrow \prod_{g \in G} H / H_{1}^{g}$ defined by $\theta(h)=\left(h H_{1}^{g}\right)_{g \in G}$ for each $h \in H$. It follows that $H / \bigcap_{g \in G} H_{1}^{g}$ can be embedded in $\prod_{g \in G} H / H_{1}^{g}$. Hence $H / \bigcap_{g \in G} H_{1}^{g}$ is abelian. Replacing $H_{1}$ by $\bigcap_{g \in G} H_{1}^{g}$, we may assume that $H_{1}$ is normal in $G$. Now, we replace $H_{1}$, if necessary, by a larger subgroup of $H$ to assume that $H_{1}$ is a maximal subgroup of $H$ with the property that it is normal in $G$ and $H / H_{1}$ is non-trivial abelian. Lifting elements of $\Gamma$ via $\alpha$ followed by conjugation gives rise to an action of $\Gamma$ on $H / H_{1}$. 
Hence $H / H_{1}$ becomes a simple $\Gamma$-module. Therefore $H / H_{1}$ becomes a simple $\operatorname{Gal}\left(K_{0}\right)$-module via $\rho$. Thus there exist a prime number $l_{1}$ and a positive integer $r_{1}$ such that $H / H_{1} \cong C_{l_{1}}^{r_{1}}$.

Note that $|H|=\left|H / H_{1}\right| \cdot\left|H_{1}\right|$. Hence by (4.2.1), we have

$$
\Omega(|H|)=\Omega\left(\left|H / H_{1}\right|\right)+\Omega\left(\left|H_{1}\right|\right) .
$$

Using Proposition 4.2.2, we first solve the step:

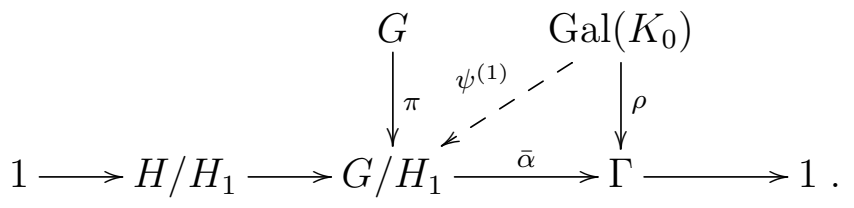

After that we apply induction on the order of $H$ in the step

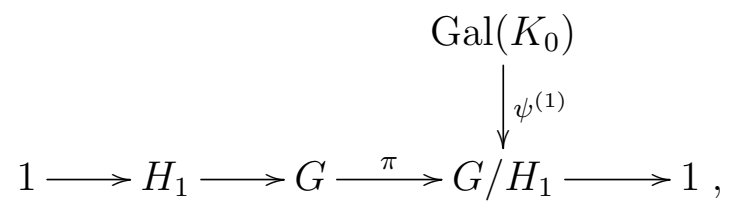

and show that the obtained solution in this step is the desired solution of the original embedding problem 4.3.1.

Theorem 4.3.2. Let $K / K_{0}$ be a finite Galois extension of number fields and consider the finite embedding problem (4.3.1) with a solvable kernel $H$, where $\Gamma=\operatorname{Gal}\left(K / K_{0}\right)$ and $\rho$ being the restriction map. Let $T$ be a finite set of primes of $K_{0}$ such that $\operatorname{Ram}\left(K / K_{0}\right) \subseteq T$. Suppose $\operatorname{gcd}(|H|,|\mu(K)|)=1$, and $\prod_{\mathfrak{p}} \mathcal{H} \operatorname{om}_{\Gamma, \rho_{\mathfrak{p}}, \alpha}\left(\operatorname{Gal}\left(\hat{K}_{0, \mathfrak{p}}\right), G\right) \neq \emptyset$. For each $\mathfrak{p} \in T$, let $\left[\varphi_{\mathfrak{p}}\right] \in \mathcal{H} \mathrm{om}_{\Gamma, \rho_{\mathfrak{p}}, \alpha}\left(\operatorname{Gal}\left(\hat{K}_{0, \mathfrak{p}}\right), G\right)$. Then there exists an element $[\psi] \in \mathcal{H}_{\Gamma, \rho, \alpha}\left(\operatorname{Gal}\left(K_{0}\right), G\right)_{\text {sur }}$ and there exists a set $R \subset \mathbb{P}\left(K_{0}\right) \backslash T$ with $|R|=\Omega(|H|)$ such that

(a) $\left[\psi_{\mathfrak{p}}\right]=\left[\varphi_{\mathfrak{p}}\right]$ in $\mathcal{H}_{\Gamma, \rho_{\mathfrak{p}}, \alpha}\left(\operatorname{Gal}\left(\hat{K}_{0, \mathfrak{p}}\right), G\right)$ for each $\mathfrak{p} \in T$,

(b) $[\psi]$ is unramified at each $\mathfrak{p} \in \mathbb{P}\left(K_{0}\right) \backslash(T \bullet R)$, that is, if $N$ is the fixed field of $\operatorname{Ker}(\psi)$, then $\operatorname{Ram}\left(N / K_{0}\right) \subseteq T \bullet R$. 
Proof. Let $H_{1}$ be the normal subgroup of $G$ contained in $H$ with $H / H_{1}$ being a simple $G$-module constructed in Construction 4.3.1. The proof breaks up into three parts.

Part A: An embedding problem whose kernel is a simple $\mathrm{Gal}\left(K_{0}\right)$-module. Let $\pi: G \longrightarrow G / H_{1}$ be the quotient map. Then, there exists an epimorphism $\bar{\alpha}: G / H_{1} \longrightarrow \Gamma$ with $\bar{\alpha} \circ \pi=\alpha$. Consider the following embedding problem:

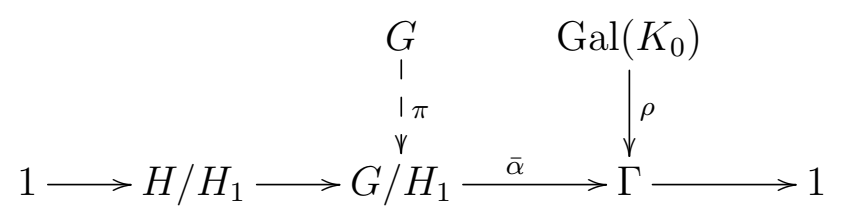

Let $\mathcal{H} \mathrm{om}_{\Gamma, \rho_{\mathfrak{p}}, \bar{\alpha}}\left(\operatorname{Gal}\left(\hat{K}_{0, \mathfrak{p}}\right), G / H_{1}\right)$ be the set of the equivalence classes corresponding to the diagram

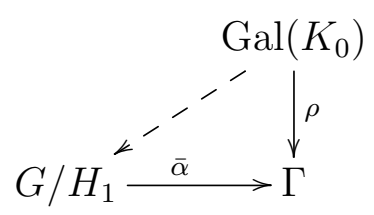

For each $\mathfrak{p} \in \mathbb{P}\left(K_{0}\right)$, if $\left[\eta_{\mathfrak{p}}\right] \in \mathcal{H} \operatorname{om}_{\Gamma, \rho_{\mathfrak{p}}, \alpha}\left(\operatorname{Gal}\left(\hat{K}_{0, \mathfrak{p}}\right), G\right)$ and $\bar{\eta}_{\mathfrak{p}}=\pi \circ \eta_{\mathfrak{p}}$, then the following diagram commutes:

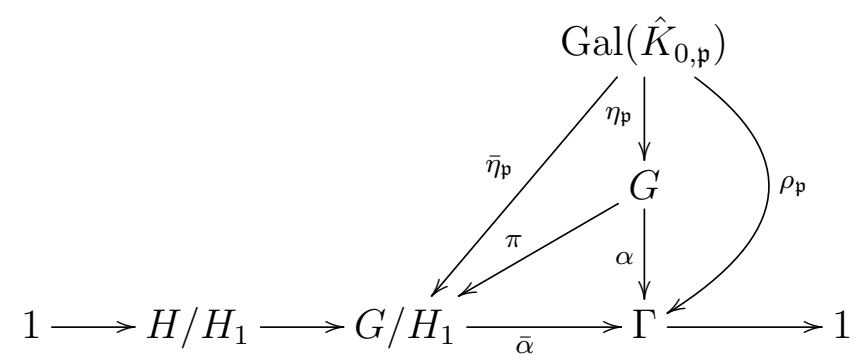

It follows that $\left[\bar{\eta}_{\mathfrak{p}}\right] \in \mathcal{H}_{\Gamma, \rho_{\mathfrak{p}}, \bar{\alpha}}\left(\operatorname{Gal}\left(\hat{K}_{0, \mathfrak{p}}\right), G / H_{1}\right)$. Hence

$$
\prod_{\mathfrak{p}} \mathcal{H} \mathrm{om}_{\Gamma, \rho_{\mathfrak{p}}, \bar{\alpha}}\left(\operatorname{Gal}\left(\hat{K}_{0, \mathfrak{p}}\right), G / H_{1}\right) \neq \emptyset
$$

For each $\mathfrak{p} \in T$, consider the element $\left[\bar{\varphi}_{\mathfrak{p}}\right] \in \mathcal{H}_{\Gamma, \rho_{\mathfrak{p}}, \bar{\alpha}}\left(\operatorname{Gal}\left(\hat{K}_{0, \mathfrak{p}}\right), G / H_{1}\right)$ with $\bar{\varphi}_{\mathfrak{p}}=\pi \circ \varphi_{\mathfrak{p}}$. 
Since $|H|$ is a multiple of $l_{1}\left|H_{1}\right|$ and $\operatorname{gcd}(|H|,|\mu(K)|)=1$, Proposition 4.2 .2 (taking $n=|H|$ ) provides a set $T_{1} \subseteq \mathbb{P}\left(K_{0}\right) \backslash T$ of order $\Omega\left(\left|H / H_{1}\right|\right)$ and an element $\left[\psi^{(1)}\right] \in \mathcal{H}_{\Gamma, \rho, \bar{\alpha}}\left(\operatorname{Gal}\left(K_{0}\right), G / H_{1}\right)_{\text {sur }}$ such that

$$
\left[\psi_{\mathfrak{p}}^{(1)}\right]=\left[\bar{\varphi}_{\mathfrak{p}}\right] \text { in } \mathcal{H} \operatorname{om}_{\Gamma, \rho_{\mathfrak{p}}, \bar{\alpha}}\left(\operatorname{Gal}\left(\hat{K}_{0, \mathfrak{p}}\right), G / H_{1}\right) \text { for each } \mathfrak{p} \in T
$$

(4.3.5) $\left[\psi^{(1)}\right]$ is unramified at each $\mathfrak{p} \in \mathbb{P}\left(K_{0}\right) \backslash\left(T \bullet T_{1}\right)$, that is if $N^{(1)}$ is the fixed field of $\operatorname{Ker}\left(\psi^{(1)}\right)$ then $\operatorname{Ram}\left(N^{(1)} / K_{0}\right) \subseteq T \bullet T_{1}$,

(4.3.6) for each $\mathfrak{p} \in \mathbb{P}\left(K_{0}\right) \backslash T$, we may lift $\left[\psi_{\mathfrak{p}}^{(1)}\right]$ to an element of $\mathcal{H o m}_{G / H_{1}, \psi_{\mathfrak{p}}^{(1)}, \pi}\left(\operatorname{Gal}\left(\hat{K}_{0, \mathfrak{p}}\right), G\right)$, and

(4.3.7) $\operatorname{gcd}\left(|H|,\left|\mu\left(N^{(1)}\right)\right|\right)=1$.

Part B: The induction step. This gives rise to an embedding problem:

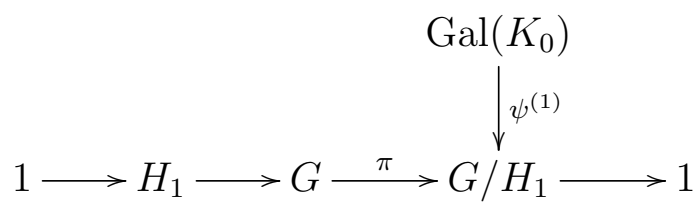

with a finite solvable kernel. Consider the set $\mathcal{H}_{\mathrm{om}_{G / H_{1}, \psi_{\mathfrak{p}}^{(1)}, \pi}}\left(\operatorname{Gal}\left(\hat{K}_{0, \mathfrak{p}}\right), G\right)$ which corresponds to the diagram

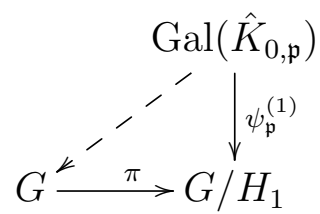

For each $\mathfrak{p} \in T$, by (4.3.4), there exists $a_{\mathfrak{p}} \in H$, such that for each $\sigma \in$ $\operatorname{Gal}\left(\hat{K}_{0, \mathfrak{p}}\right)$

$\psi_{\mathfrak{p}}^{(1)}(\sigma)=\pi\left(a_{\mathfrak{p}}\right)^{-1} \bar{\varphi}(\sigma) \pi\left(a_{\mathfrak{p}}\right)=\pi\left(a_{\mathfrak{p}}\right)^{-1}\left(\pi \circ \varphi_{\mathfrak{p}}\right)(\sigma) \pi\left(a_{\mathfrak{p}}\right)=\pi\left(a_{\mathfrak{p}}^{-1} \varphi_{\mathfrak{p}}(\sigma) a_{\mathfrak{p}}\right)=\pi \circ \varphi_{\mathfrak{p}}^{a_{\mathfrak{p}}}(\sigma)$.

(4.3.9) So $\left[\varphi_{\mathfrak{p}}^{a_{\mathfrak{p}}}\right] \in \mathcal{H}_{\mathrm{om}}{ }_{G / H_{1}, \psi_{\mathfrak{p}}^{(1)}, \pi}\left(\operatorname{Gal}\left(\hat{K}_{0, \mathfrak{p}}\right), G\right)$ for each $\mathfrak{p} \in T$.

It follows from (4.3.6) and (4.3.9) that $\prod_{\mathfrak{p}} \mathcal{H o m}_{G / H_{1}, \psi_{\mathfrak{p}}^{(1)}, \pi}\left(\operatorname{Gal}\left(\hat{K}_{0, \mathfrak{p}}\right), G\right) \neq \emptyset$. Moreover, (4.3.7) implies that $\operatorname{gcd}\left(\left|H_{1}\right|,\left|\mu\left(N^{(1)}\right)\right|\right)=1$. 
Consider the system $\left(\left[\varphi_{\mathfrak{p}}^{(1)}\right] \in \mathcal{H}^{\left(0 m / H_{1}, \psi_{\mathfrak{p}}^{(1)}, \pi\right.}{ }\left(\operatorname{Gal}\left(\hat{K}_{0, \mathfrak{p}}\right), G\right)\right)_{\mathfrak{p} \in T \cup T_{1}}$, such that if $\mathfrak{p} \in T$ then $\varphi_{\mathfrak{p}}^{(1)}=\varphi_{\mathfrak{p}}^{a_{\mathfrak{p}}}$, and if $\mathfrak{p} \in T_{1}$ we use (4.3.6) to choose $\left[\varphi_{\mathfrak{p}}^{(1)}\right]$.

Since $H_{1}$ is solvable and $\left|H_{1}\right|<|H|$, our induction hypothesis provides a set $R_{1} \subset \mathbb{P}\left(K_{0}\right) \backslash\left(T \bullet T_{1}\right)$ with $\left|R_{1}\right|=\Omega\left(\left|H_{1}\right|\right)$, and an element $[\psi] \in$ $\mathcal{H o m}_{G / H_{1}, \psi^{(1)}, \pi}\left(\operatorname{Gal}\left(K_{0}\right), G\right)_{\text {sur }}$ such that

(4.3.10) for each $\mathfrak{p} \in T \bullet T_{1},\left[\psi_{\mathfrak{p}}\right]=\left[\varphi_{\mathfrak{p}}^{(1)}\right]$ in $\mathcal{H} \mathrm{om}_{G / H_{1}, \psi_{\mathfrak{p}}^{(1)}, \pi}\left(\operatorname{Gal}\left(\hat{K}_{0, \mathfrak{p}}\right), G\right)$,

(4.3.11) $[\psi]$ is unramified at each $\mathfrak{p} \in \mathbb{P}\left(K_{0}\right) \backslash\left(T \bullet T_{1} \bullet R_{1}\right)$, that is if $N$ is the solution field then $\operatorname{Ram}\left(N / K_{0}\right) \subseteq T \bullet T_{1} \bullet R_{1}$.

We set $R=T_{1} \bullet R_{1}$. Then $|R|=\left|T_{1}\right|+\left|R_{1}\right|=\Omega\left(\left|H / H_{1}\right|\right)+\Omega\left(\left|H_{1}\right|\right)=\Omega(|H|)$ (See 4.3.2).

Part C: Conclusion of the proof. Let us prove that $[\psi]$ satisfies the conclusion of the Theorem. Indeed, $\pi \circ \psi=\psi^{(1)}$, so $\bar{\alpha} \circ \pi \circ \psi=\bar{\alpha} \circ \psi^{(1)}$. Since $\bar{\alpha} \circ \pi=\alpha$ and $\bar{\alpha} \circ \psi^{(1)}=\rho$, we have $\alpha \circ \psi=\rho$, that is $[\psi] \in \mathcal{H}_{\Gamma, \rho, \alpha}\left(\operatorname{Gal}\left(K_{0}\right), G\right)_{\text {sur }}$.

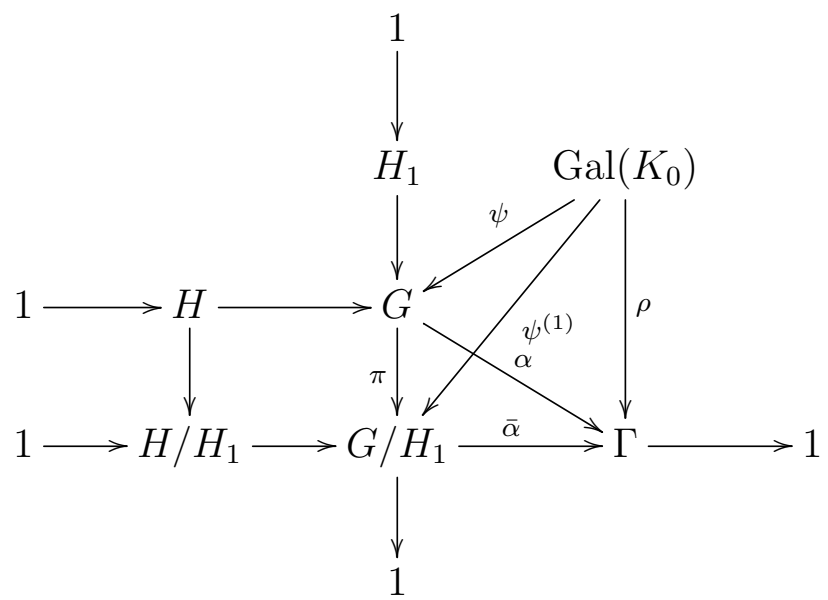

Furthermore, by (4.3.10), for each $\mathfrak{p} \in T$, there exists $b_{\mathfrak{p}} \in H_{1}$ such that for 
each $\sigma \in \operatorname{Gal}\left(\hat{K}_{0, \mathfrak{p}}\right)$

$$
\begin{aligned}
\psi_{\mathfrak{p}}(\sigma) & =\left(\varphi_{\mathfrak{p}}^{(1)}\right)^{b_{\mathfrak{p}}}(\sigma) \\
& =b_{\mathfrak{p}}^{-1} a_{\mathfrak{p}}^{-1} \varphi_{\mathfrak{p}}(\sigma) a_{\mathfrak{p}} b_{\mathfrak{p}} \\
& =\left(a_{\mathfrak{p}} b_{\mathfrak{p}}\right)^{-1} \varphi_{\mathfrak{p}}(\sigma)\left(a_{\mathfrak{p}} b_{\mathfrak{p}}\right) \\
& =\varphi_{\mathfrak{p}}^{a_{\mathfrak{p}} b_{\mathfrak{p}}}(\sigma)
\end{aligned}
$$

Since $a_{\mathfrak{p}} \in H, b_{\mathfrak{p}} \in H_{1}$, we have $a_{\mathfrak{p}} b_{\mathfrak{p}} \in H$. Therefore $\left[\psi_{\mathfrak{p}}\right]=\left[\varphi_{\mathfrak{p}}\right]$ in $\mathcal{H} \mathrm{om}_{\Gamma, \rho_{\mathfrak{p}}, \alpha}\left(\operatorname{Gal}\left(\hat{K}_{0, \mathfrak{p}}\right), G\right)$ for each $\mathfrak{p} \in T$, as desired.

\subsection{Solving Embedding Problems with Solvable Kernels in $K_{0, \text { tot }, S}$}

In this section we give some applications of the main theorem of this work. Let $K_{0}$ be a number field and $S$ a finite set of primes of $K_{0}$. Let $K_{0, \text { tot, } S}$ be the maximal Galois extension of $K_{0}$ where each prime $\mathfrak{p} \in S$ totally splits. By definition

$$
K_{0, \text { tot }, S}=\bigcap_{\mathfrak{p} \in S} \bigcap_{\sigma \in \operatorname{Gal}\left(K_{0}\right)} K_{0, \mathfrak{p}}^{\sigma}
$$

where $K_{0, \mathfrak{p}}$ is a henselization of $K_{0}$ at $\mathfrak{p}$ (the algebraic part of $\hat{K}_{0, \mathfrak{p}}$ ).

In particular, for a prime $p$ we write $\mathbb{Q}_{\text {tot }, p}$ rather than $\mathbb{Q}_{\text {tot },\{p\}}$. In this case the thesis [Rami13] conjectures that every finite group that can be realized over $\mathbb{Q}$ can also be realized over $\mathbb{Q}$ in $\mathbb{Q}_{\text {tot }, p}$. We proved there that every abelian group, symmetric group, and alternating group can be realized over

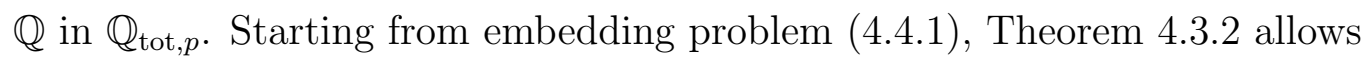
us now to solve it with local data and bounded ramification in $K_{0, \text { tot, } S}$ once the kernel $H$ is solvable, $\operatorname{gcd}(|H|,|\mu(K)|)=1, K \subset K_{0, \text { tot }, S}$, and each of the corresponding local embedding problem is solvable. In particular, the latter result leads to the realization of every solvable group over $K_{0}$ in $K_{0, \text { tot, } S}$ with bounded ramification, and to the solvability of each split embedding problem 
in $K_{0, \text { tot }, S}$ under the above necessary condition.

Let $K / K_{0}$ be a finite Galois extension of number fields with $\operatorname{Gal}\left(K / K_{0}\right)=\Gamma$. Set $\rho=\operatorname{res}_{\tilde{K}_{0} / K}$. Consider the finite embedding problem

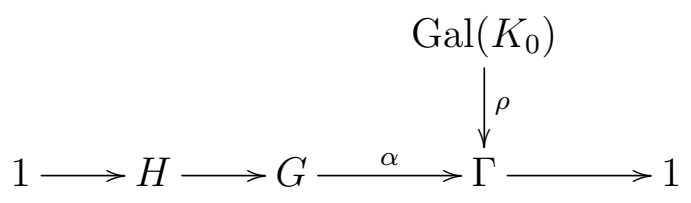

with $H$ a solvable group. Under the conditions of Theorem 4.3.2, if $K \subset$ $K_{0, \text { tot,S }}$, then the embedding problem has a proper solution in $K_{0, \text { tot,S }}$ with bounded ramification.

Theorem 4.4.1. Let $K / K_{0}$ be a finite Galois extension of number fields, and let $S$ be a finite set of primes of $K_{0}$ such that $K \subseteq K_{0, \text { tot }, S}$. Consider embedding problem (4.4.1) with a solvable kernel $H$, where $\Gamma=\operatorname{Gal}\left(K / K_{0}\right)$ and $\rho$ being the restriction map. Suppose $\operatorname{gcd}(|H|,|\mu(K)|)=1$, and $\prod_{\mathfrak{p}} \mathcal{H} \operatorname{om}_{\Gamma, \rho_{\mathfrak{p}}, \alpha}\left(\operatorname{Gal}\left(\hat{K}_{0, \mathfrak{p}}\right), G\right) \neq \emptyset$. Let $T \subset \mathbb{P}\left(K_{0}\right) \backslash S$ be a finite set of primes such that $\operatorname{Ram}\left(K / K_{0}\right) \subseteq T$. For each $\mathfrak{p} \in T$, let $\left[\varphi_{\mathfrak{p}}\right] \in \mathcal{H}_{\Gamma, \rho_{\mathfrak{p}}, \alpha}\left(\operatorname{Gal}\left(\hat{K}_{0, \mathfrak{p}}\right), G\right)$. There exists an element $[\psi] \in \mathcal{H}_{\Gamma, \rho, \alpha}\left(\operatorname{Gal}\left(K_{0}\right), G\right)_{\text {sur }}$ and there exists a set $R \subseteq \mathbb{P}\left(K_{0}\right) \backslash(T \bullet S)$ with $|R|=\Omega(|H|)$, such that if we denote $\left[\psi_{\mathfrak{p}}\right]=$ $\left[\left.\psi\right|_{\operatorname{Gal}\left(\hat{K}_{0, \mathfrak{p}}\right)}\right]$ then

(a) $\left[\psi_{\mathfrak{p}}\right]=\left[\varphi_{\mathfrak{p}}\right]$ for each $\mathfrak{p} \in T$,

(b) the fixed field $N$ of $\operatorname{Ker}(\psi)$ satisfies $N \subset K_{0, \text { tot }, S}$,

(c) $\operatorname{Ram}\left(N / K_{0}\right) \subseteq T \bullet R$

Proof. Since $K \subset K_{0, \text { tot }, S}$, each $\mathfrak{p} \in S$ totally splits in $K$, hence $\mathfrak{p}$ is unramified in $K$ and $\rho_{\mathfrak{p}}: \operatorname{Gal}\left(\hat{K}_{0, \mathfrak{p}}\right) \rightarrow \Gamma$ is the trivial homomorphism. Thus, the equivalence class of the trivial homomorphism $\varphi_{\mathfrak{p}}: \operatorname{Gal}\left(\hat{K}_{0, \mathfrak{p}}\right) \rightarrow G$ is an element of $\mathcal{H}_{\Gamma, \rho_{\mathfrak{p}}, \alpha}\left(\operatorname{Gal}\left(\hat{K}_{0, \mathfrak{p}}\right), G\right)$. Let $T_{1}=T \bullet S$ and consider the system $\left(\left[\varphi_{\mathfrak{p}}\right] \in \mathcal{H} \operatorname{om}_{\Gamma, \rho_{\mathfrak{p}}, \alpha}\left(\operatorname{Gal}\left(\hat{K}_{0, \mathfrak{p}}\right), G\right)\right)_{\mathfrak{p} \in T_{1}}$. 
By Theorem 4.3.2, there exists an element $[\psi] \in \mathcal{H}_{\Gamma, \rho, \alpha}\left(\operatorname{Gal}\left(K_{0}\right), G\right)_{\text {sur }}$ and there exists an set $R \subset \mathbb{P} \backslash T_{1}$ with $|R|=\Omega(|H|)$ such that

(4.4.2) for each $\mathfrak{p} \in T_{1},\left[\psi_{\mathfrak{p}}\right]=\left[\varphi_{\mathfrak{p}}\right]$ in $\mathcal{H} \mathrm{om}_{\Gamma, \rho_{\mathfrak{p}}, \alpha}\left(\operatorname{Gal}\left(\hat{K}_{0, \mathfrak{p}}\right), G\right)$,

(4.4.3) the solution field $N$ (fixed field of $\operatorname{Ker}(\psi))$ satisfies $\operatorname{Ram}\left(N / K_{0}\right) \subseteq$ $T_{1} \bullet R$

Proof of (a). Since $T \subset T_{1}$, (a) follows from (4.4.2).

Proof of (b). For each $\mathfrak{p} \in S$, by $\left[(4.4 .2)\right.$, $\left[\psi_{\mathfrak{p}}\right]=\left[\varphi_{\mathfrak{p}}\right]$. Since $\varphi_{\mathfrak{p}}$ is the trivial homomorphism, so is $\psi_{\mathfrak{p}}$. It follows that each $\mathfrak{p} \in S$ totally splits in $N$. Thus $N \subseteq K_{0, \text { tot }, S}$.

Proof of (c). By (a), each prime $\mathfrak{p} \in S$ totally splits in $N$. Since $T_{1}=T \bullet S$, it follows from (4.4.3) that $\operatorname{Ram}\left(N / K_{0}\right) \subseteq T \bullet R$.

An immediate consequence of Theorem 4.4.1 is that every solvable group whose order is prime to $\left|\mu\left(K_{0}\right)\right|$ can be realized over $K_{0}$ in $K_{0, \text { tot, } S}$ with bounded ramification.

Corollary 4.4.2. Let $K_{0}$ be a number field and let $S$ be a finite set of primes of $K_{0}$. Let $G$ be a finite solvable group with $\operatorname{gcd}\left(|G|,\left|\mu\left(K_{0}\right)\right|\right)=1$. Then, there exists a Galois extension $N / K_{0}$ with $N \subseteq K_{0, \text { tot }, S}$ and $\operatorname{Gal}\left(N / K_{0}\right) \cong G$ such that $\left|\operatorname{Ram}\left(N / K_{0}\right)\right| \leq \Omega(|G|)$.

Proof. We set $\Gamma=1$ the trivial group, and we consider the following embedding problem:

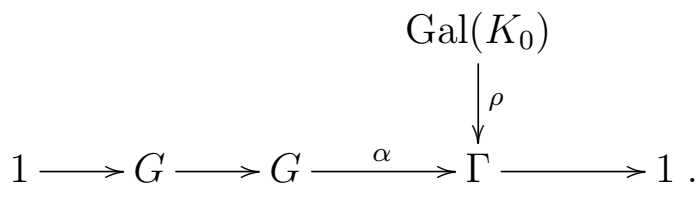

with $\alpha$ the trivial epimorphism. Then, for each prime $\mathfrak{p} \in \mathbb{P}\left(K_{0}\right)$, the class of the trivial homomorphism $\varphi_{p}: \operatorname{Gal}\left(\hat{K}_{0, \mathfrak{p}}\right) \longrightarrow G$ is an element of 
$\mathcal{H o m}_{\Gamma, \rho_{\mathfrak{p}}, \alpha}\left(\operatorname{Gal}\left(\hat{K}_{0, \mathfrak{p}}\right), G\right)$, so

$$
\prod_{\mathfrak{p}} \mathcal{H} \mathrm{om}_{\Gamma, \rho_{\mathfrak{p}}, \alpha}\left(\operatorname{Gal}\left(\hat{K}_{0, \mathfrak{p}}\right), G\right) \neq \emptyset
$$

Considering $K=K_{0}, T=\{\mathfrak{p}\}$ for some $\mathfrak{p} \in \mathbb{P}\left(K_{0}\right)$, and $\left[\varphi_{\mathfrak{p}}\right] \in \mathcal{H}_{\Gamma, \rho_{\mathfrak{p}}, \alpha}\left(\operatorname{Gal}\left(\hat{K}_{0, \mathfrak{p}}\right), G\right)$ the class of the trivial homomorphism, by Theorem 4.4.1, there exists a Galois extension $N / K_{0}$ with $N \subseteq K_{0, \text { tot }, S}$ such that $\operatorname{Gal}\left(N / K_{0}\right) \cong G$ and $\left|\operatorname{Ram}\left(N / K_{0}\right)\right| \leq \Omega(|G|)$.

The next application of Theorem 4.4.1 says that if embedding problem 4.4.1 has a solution in $K_{0, \text { tot, } S}$, then it has a proper solution in $K_{0, \text { tot, } S}$ with bounded ramification.

Corollary 4.4.3. Let $K / K_{0}$ be a finite Galois extension of number fields

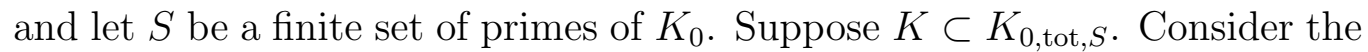
embedding problem 4.4.1 with a solvable kernel $H$, where $\Gamma=\operatorname{Gal}\left(K / K_{0}\right)$ and $\rho$ being the restriction map. Suppose $\operatorname{gcd}(|H|,|\mu(K)|)=1$. If the embedding problem 4.4.1) has a solution $\psi_{0}$ (homomorphism) in $K_{0, \text { tot, } S}$ then it has a proper solution $\psi$ (epimorphism) in $K_{0, \text { tot }, S}$ with $|\operatorname{Ram}(\psi)| \leq$ $\left|\operatorname{Ram}\left(K / K_{0}\right)\right|+\Omega(|H|)$.

Proof. For each $\mathfrak{p} \in \mathbb{P}\left(K_{0}\right)$ the local restriction $\psi_{0, \mathfrak{p}}: \operatorname{Gal}\left(\hat{K}_{0, \mathfrak{p}}\right) \rightarrow G$ is a solution of the corresponding local embedding problem. It follows that $\prod_{\mathfrak{p}} \mathcal{H} \mathrm{om}_{\Gamma, \rho_{\mathfrak{p}}, \alpha}\left(\operatorname{Gal}\left(\hat{K}_{0, \mathfrak{p}}\right), G\right) \neq \emptyset$. Therefore, by Theorem 4.4.1. considering $T=\operatorname{Ram}\left(K / K_{0}\right)$, there exists a proper solution (epimorphism) $\psi: \operatorname{Gal}\left(K_{0}\right) \rightarrow$ $G$ in $K_{0, \text { tot }, S}$ with $|\operatorname{Ram}(\psi)| \leq\left|\operatorname{Ram}\left(K / K_{0}\right)\right|+\Omega(|H|)$.

Always assuming that $K \subset K_{0, \text { tot, } S}$, Corollary 4.4.3 implies that if the embedding problem (4.4.1) splits, i.e. $\alpha$ has a section, then it has a proper solution in $K_{0, \text { tot, } S}$ with bounded ramification.

Corollary 4.4.4. Let $K / K_{0}$ be a finite Galois extension of number fields and let $S$ be a finite set of primes of $K_{0}$. Suppose $K \subset K_{0, \text { tot }, S}$. Consider embedding problem 4.4.1 with a solvable kernel $H$, where $\Gamma=\operatorname{Gal}\left(K / K_{0}\right)$ 
and $\rho$ being the restriction map. Suppose $\operatorname{gcd}(|H|,|\mu(K)|)=1$ and the epimorphism $\alpha$ splits. Then the embedding problem 4.4.1) has a proper solution $\psi$ in $K_{0, \text { tot }, S}$ with $|\operatorname{Ram}(\psi)| \leq\left|\operatorname{Ram}\left(K / K_{0}\right)\right|+\Omega(|H|)$.

Proof. Let $s: \Gamma \rightarrow G$ be a section of $\alpha$, i.e. $\alpha \circ s=\mathrm{id}_{\Gamma}$. Then, the homomorphism $\psi_{0}=s \circ \rho: \operatorname{Gal}\left(K_{0}\right) \rightarrow G$ is a solution of 4.4.1) in $K_{0, \text { tot }, S}$. Therefore, our corollary is a special case of Corollary 4.4.3. 


\section{Bibliography}

[CaF67] J. W. Cassels and A. Fröhlich, Algebraic Number Theory, Academic Press, London (1967).

[FrJ08] M. D. Fried and M. Jarden, Field Arithmetic, third edition,revised by M. Jarden, Ergebnisse der Mathematik (3) 11, Springer, Heidelberg (2008).

[GeJ98] W. Geyer and M. Jarden, Bounded realization of l-groups over global fields, Nagoya Mathematical Journal 150 (1998), pp. 13-62.

[HaW62] G. H. Hardy and E. M. Wright, An Introduction to the Theory of Numbers, Fourth Edition, Oxford (1962)

[Jan73] G. Janusz, Algebraic Number Fields, Academic Press, New York (1973).

[Jar91] M. Jarden, Intersection of local algebraic extensions of a Hilbertian field, NATO ASI Series C 333 (1991), 343-405.

[Lan70] S. Lang, Algebraic Number Theory, Addison-Wesley, Reading (1970).

[Lan93] S. Lang, Algebra, third edition, Addison-Wesley, Reading (1993).

[MaU11] N. Markin and S.-V. Ullom, Minimal ramification in nilpotent extensions, Pacific Journal of Mathematics 253 (2011), No. 1, pp. 125-143. 
[Neu79] J. Neukirch, On solvable number fields, Inventiones mathematicae 53 (1979), pp. 135-164.

[Neu99] J. Neukirch, Algebraic Number Theory, Springer-Verlag, Heidelberg (1999).

[NSW00] J. Neukirch, A. Schmidt, and K. Wingberg, Cohomology of Number Fields, Grundlehren der mathematischen Wissenschaften 323, Springer-Verlag, Heidelberg (2000).

[Rami13] C. Ramiharimanana, Realization of finite groups as Galois groups over $\mathbb{Q}$ in $\mathbb{Q}_{\text {tot, } p}$, Master Thesis, Stellenbosch University (2013).

[Rei37] H. Reichardt, Konstruktion von zahlkörpern mit gegebener Galoisgruppe von primzahlpotenzordnung, Journal für die reine und angewandte Mathematik 177 (1937), pp. 1-6.

[Rib70] L. Ribes, Introduction to Profinite Groups and Galois Cohomology, Queen's papers in Pure and Applied Mathematics 24, Queen's University, Kingston (1970).

[Sch37] A. Scholz, Konstruktion algebraischer Zahlkörper mit beliebiger gruppe von primzahlpotenzordnung I., Mathematische Zeitschrift 42 (1937), pp. 161-188.

[Se92] J.-P. Serre, Topics in Galois Theory, Jones and Barlett, Boston (1992).

[Se79] J.-P. Serre, Local Fields, Springer-Verlag, New York (1979), Translation from French by M. J. Greenberg.

[Sha54] I. R. Shafarevich, On the construction of fields with a given galois group of order $l^{a}$, Izv. Akad. Nauk 18 (1954), pp. 261-296. In Russian. AMS Translations, Ser. 2, 4 (1956), pp. 107-142.

[Sha89] I. R. Shafarevich, Factors of descending central series, Mathematical Notes 45 (1989), pp. 262-264. 